\title{
Characterisation of 92 Southern TESS Candidate Planet Hosts and a New Photometric [Fe/H] Relation for Cool Dwarfs
}

\author{
Adam D. Rains, ${ }^{1 \star}$ Maruša Žerjal, ${ }^{1}$ Michael J. Ireland, ${ }^{1}$ Thomas Nordlander, ${ }^{1,2}$ \\ Michael S. Bessell, ${ }^{1}$ Luca Casagrande, ${ }^{1,2}$ Christopher A. Onken, ${ }^{1,3}$ Meridith Joyce, ${ }^{1,2}$ \\ Jens Kammerer, ${ }^{1,4}$ and Harrison Abbot $^{1}$ \\ ${ }^{1}$ Research School of Astronomy and Astrophysics, Australian National University, Canberra, ACT 2611, Australia \\ ${ }^{2}$ ARC Centre of Excellence for All Sky Astrophysics in 3 Dimensions (ASTRO 3D) \\ ${ }^{3}$ Centre for Gravitational Astrophysics, Research Schools of Physics, and Astronomy and Astrophysics, Australian National University \\ ${ }^{4}$ European Southern Observatory, Karl-Schwarzschild-Str 2, 85748, Garching, Germany
}

Last updated 2015 May 22; in original form 2013 September 5

\begin{abstract}
We present the results of a medium resolution optical spectroscopic survey of $92 \operatorname{cool}(3,000 \lesssim$ $\left.T_{\text {eff }} \lesssim 4,500 \mathrm{~K}\right)$ southern TESS candidate planet hosts, and describe our spectral fitting methodology used to recover stellar parameters. We quantify model deficiencies at predicting optical fluxes, and while our technique works well for $T_{\text {eff }}$, further improvements are needed for $[\mathrm{Fe} / \mathrm{H}]$. To this end, we developed an updated photometric $[\mathrm{Fe} / \mathrm{H}]$ calibration for isolated main sequence stars built upon a calibration sample of 69 cool dwarfs in binary systems, precise to $\pm 0.19 \mathrm{dex}$, from super-solar to metal poor, over $1.51<\mathrm{Gaia}\left(B_{P}-R_{P}\right)<3.3$. Our fitted $T_{\text {eff }}$ and $R_{\star}$ have median precisions of $0.8 \%$ and $1.7 \%$, respectively and are consistent with our sample of standard stars. We use these to model the transit light curves and determine exoplanet radii for 100 candidate planets to $3.5 \%$ precision and see evidence that the planetradius gap is also present for cool dwarfs. Our results are consistent with the sample of confirmed TESS planets, with this survey representing one of the largest uniform analyses of cool TESS candidate planet hosts to date.
\end{abstract}

Key words: stars: low-mass, stars: fundamental parameters, planets and satellites: fundamental parameters, techniques: spectroscopic,

\section{INTRODUCTION}

Low mass stars are the most common kind of star in the Galaxy, comprising more than two thirds of all stars (Chabrier 2003), and dominating the Solar Neighbourhood population (e.g. Henry et al. 1994, 2006; Winters et al. 2015; Henry et al. 2018). This abundance alone makes them prime targets for planet searches, with microlensing surveys, which have very little bias on host star masses, revealing that there is at least one bound planet per Milky Way star (Cassan et al. 2012). Results from the Kepler Mission (Borucki et al. 2010) also bear this out, showing that a large number of planets remain undiscovered around cool dwarfs (Morton \& Swift 2014), and that such cool stars are actually more likely to host small planets $\left(2<R_{P}<4 R_{\oplus}\right.$, where $R_{P}$ and $R_{\oplus}$ are the planet and earth radius respectively) than their hotter counterparts (Howard et al. 2012; Dressing \& Charbonneau 2015).

However, the inherent faintness of these stars complicates the study of both them and their planets. While we now know of over
4,000 confirmed planets orbiting stars other than our own (overwhelmingly discovered by transiting exoplanet surveys), almost an equal number await confirmation ${ }^{1}$. Exoplanet transit surveys like Kepler and TESS (Ricker et al. 2015) are able to place tight constraints on planetary radii given a known stellar radius, but followup precision radial velocity observations are required to provide planetary mass constraints. This is the second reason why planet searches around low mass stars are critical: their smaller radii and lower masses make the transit signals and radial velocities of higher amplitudes for any planets they host as compared to the same planets around more massive host stars. This is especially important when looking for planets with terrestrial radii or masses respectively.

Many planet host stars have never been targeted by a spectroscopic survey, leaving their properties to be estimated through photometry alone. For instance, the TESS input catalogue (Stassun et al. 2018,2019) based its stellar parameters primarily on photometry, having spectroscopic properties for only about 4 million stars of the nearly 700 million with photometrically estimated equivalents. 
While stars warmer than $4,000 \mathrm{~K}$ are well suited to bulk estimation of properties from photometry (see e.g. Carrillo et al. 2020), special care must be taken for cool dwarfs whose faintness and complex atmospheres make such relations more complex to develop and implement (e.g. see Muirhead et al. 2018 for the K and M dwarf specific approach taken from the TESS input catalogue).

NASA's TESS Mission, by virtue of being all sky, has given us a wealth of bright candidates which are now being actively followed up by ground based spectroscopic surveys. While multi-epoch radial velocity observations are required to determine planetary masses, these surveys are typically biased towards the brightest stars and smallest planets. As such, there remains a need for single-epoch spectroscopic follow-up of fainter targets to provide reliable host star properties (primarily $T_{\mathrm{eff}}, \log g,[\mathrm{Fe} / \mathrm{H}]$, and the stellar radius $R_{\star}$ ) and allow radial constraints to be placed on transiting planet candidates. Indeed, the LAMOST Survey (Zhao et al. 2012) undertook targeted low resolution spectroscopic follow-up of stars in the Kepler field (De Cat et al. 2015) with the goal of deriving spectroscopic stellar properties. Considering the goal of planet radii determination specifically, Dressing et al. (2019) used mediumresolution near-infrared (NIR) spectra, and Wittenmyer et al. (2020) high-resolution optical spectra to follow-up K2 (Howell et al. 2014) transiting planet candidate hosts and place radius constraints on both planets and their hosts.

Even without mass estimates, much can be learned about exoplanet demographics from their radii alone. As demonstrated by Fulton et al. (2017), Fulton \& Petigura (2018), Van Eylen et al. (2018), Kruse et al. (2019), Hardegree-Ullman et al. (2020), Cloutier \& Menou (2020), and Hansen et al. (2021), having a large sample of precise planet radii allows insight into the exoplanet radius distribution, which appears to be bimodal with an observable gap in the super-Earth regime $\left(\sim 1.8 R_{\oplus}\right)$. This is thought to be the result of physical phenomena like photoevaporation (where flux from the parent star strips away weakly held atmospheres, e.g. Owen \& Wu 2013; Lee et al. 2014; Lopez \& Fortney 2014; Lee \& Chiang 2016; Owen \& Wu 2017; Lopez \& Rice 2018), or core-powered mass loss (where a cooling rocky core erodes light planetary atmospheres via its cooling luminosity, e.g. Ikoma \& Hori 2012; Ginzburg et al. 2018; Gupta \& Schlichting 2019, 2020), and its location likely has a dependence on stellar host mass (e.g. Cloutier \& Menou 2020). As such, improving the sample of planets with radius measurements allows us to place observational constraints on planet formation channels and the mechanisms that sculpt planets throughout their lives.

The scientific importance of searching for planets around lowmass stars to study their demographics is thus clear. However, the exact approach for understanding the stars themselves is less obvious, as cool dwarfs are not as well understood as their prevalence would suggest. Their inherent faintness and atmospheric complexity has lead to long standing issues observing representative sets of standard stars, generating synthetic spectra accounting for molecular absorption as well as consistently modelling their evolution (see e.g. Allard et al. 1997; Chabrier 2003).

Analysis of spectra from warmer stars is made simpler by the existence of regions of spectral continuum where atomic or molecular line absorption is minimal, allowing one to disentangle within reasonable uncertainties the effect of $[\mathrm{Fe} / \mathrm{H}]$ and $T_{\text {eff }}$ on an emerging spectrum. This is not the case for cool dwarfs for which there is no continuum at shorter wavelengths, with the deepest absorption caused by most notably $\mathrm{TiO}$ in the optical and water in the NIR, but also various other oxides or hydrides. The strength of these features is a function of both temperature and $[\mathrm{Fe} / \mathrm{H}]$, making it difficult to ascribe a unique $T_{\mathrm{eff}}-[\mathrm{Fe} / \mathrm{H}]$ pair to a given star.

Despite this complexity, it is possible to take advantage of the relative $[\mathrm{Fe} / \mathrm{H}]$-insensitivity of NIR $K$ band magnitudes alongside $[\mathrm{Fe} / \mathrm{H}]$-sensitive optical photometry to probe cool dwarf $[\mathrm{Fe} / \mathrm{H}]$. This was predicted by theory (see e.g. Allard et al. 1997, Baraffe et al. 1998, and Chabrier \& Baraffe 2000 for summaries), confirmed observationally (Delfosse et al. 2000), and later formalised into various empirical calibrations (Bonfils et al. 2005; Johnson \& Apps 2009; Schlaufman \& Laughlin 2010; Neves et al. 2012; Hejazi et al. 2015; Dittmann et al. 2016).

The last decade has seen a number of studies using low-medium resolution (mostly NIR) spectra, often focused on the development of $[\mathrm{Fe} / \mathrm{H}]$ relations based on spectral indices (e.g. optical-NIR: Mann et al. 2013b,c, 2015; Kuznetsov et al. 2019; NIR: Newton et al. 2014; H band: Terrien et al. 2012; K band: Rojas-Ayala et al. 2010, 2012). Other studies have opted to use high-resolution spectra which gives access to unblended atomic lines that are not accessible to lower resolution observations (e.g. optical: Bean et al. 2006a,b; Rajpurohit et al. 2014; Passegger et al. 2016; Y band: Veyette et al. 2017; optical-NIR: Woolf \& Wallerstein 2005, 2006; Passegger et al. 2018; J band: Önehag et al. 2012; H band: Souto et al. 2017).

Finally, on the point of M-dwarf evolutionary models (and lowmass, cool main sequence stars more generally), there has long been contention between model radii and observed radii (e.g. Kraus et al. 2015). This is often attributed to magnetic fields (and/or the mixing length parameter, which simplistically parameterizes the effects of magnetic fields among other energy transport mechanisms in 1D stellar structure and evolution programs) and is related to the difficulty in accurately modelling convection (e.g. Feiden \& Chaboyer 2012; Joyce \& Chaboyer 2018). Fortunately, due to the aforementioned insensitivity of NIR $K$ band photometry to [Fe/H], empirical mass and radius relations have been developed and calibrated on interferometric diameters and dynamical masses (e.g. Henry \& McCarthy 1993; Delfosse et al. 2000; Benedict et al. 2016; Mann et al. 2015, 2019).

Here we conduct a moderate resolution spectroscopic survey of 92 southern cool $\left(T_{\text {eff }} \lesssim 4,500 \mathrm{~K}\right)$ TESS candidate planet hosts with the WiFeS instrument (Dopita et al. 2007) on the ANU $2.3 \mathrm{~m}$ Telescope at Siding Spring Observatory (NSW, Australia). We combine our spectroscopic observations with literature optical photometry and trigonometric parallaxes from Gaia DR2 (Gaia Collaboration et al. 2016; Brown et al. 2018), infrared photometry from 2MASS (Skrutskie et al. 2006), optical photometry from SkyMapper DR3 (Keller et al. 2007, Onken et al. 2019, DR3 DOI: 10.25914/5f14eded2d116), empirical relations from (Mann et al. 2015, 2019), and synthetic MARCS model atmospheres (Gustafsson et al. 2008) in order to produce stellar $T_{\mathrm{eff}}, \log g$, [Fe/H], bolometric flux $\left(f_{\mathrm{bol}}\right), R_{\star}$, and stellar mass $\left(M_{\star}\right)$. By modelling the transit light curves of these host stars, we are additionally able to produce precision planetary radii for 100 candidate planets, which represents one of the largest uniform analyses of cool TESS hosts to date. Our observations and data reduction are described in Section 2, our photometric $[\mathrm{Fe} / \mathrm{H}]$ relation in Section 3, our host star characterisation methodology and resulting parameters in Section 4 , our transit light curve fitting and results in Section 5, discussion of results in Section 6, and concluding remarks in Section 7. 


\section{OBSERVATIONS AND DATA REDUCTION}

\subsection{Target Selection}

Our initial target selection of southern cool dwarf TOIs was done in August 2019, including stars with $T_{\text {eff }} \leqslant 4500 \mathrm{~K}$ in the TESS input catalogue and unblended 2MASS photometry. In order to have reliable parallaxes, we impose the additional requirement that our stars have a Gaia DR2 Renormalised Unit Weight Error (RUWE) $^{2}$ of $<1.4$, as recommended by the Gaia team ${ }^{3}$. Adding extra targets sourced in August 2020, and removing those identified as false-positives through community follow-up observations (as listed on NASA's Exoplanet Follow-up Observing Program for TESS, ExoFOP-TESS, website ${ }^{4}$ ), we are left with a sample of 92 southern candidate planet hosts spread across the sky with $8.7<$ apparent Gaia $G<15.8$. These targets are listed in Table 1, and plotted on a colour-magnitude diagram in Figure 1, noting that a few appear distinctly above the main sequence. These stars are thus overluminous because they are young and still contracting to the main sequence, or because they are unresolved binaries.

All our targets have Gaia DR2 $G, B_{P}, R_{P}$, and 2MASS $J, H$, $K_{S}$ photometry, and most have at least one of SkyMapper DR3 $r, i, z$ (noting that the survey is still ongoing, so not all bands are available for all targets). We calculate distances from Gaia DR2 parallaxes, incorporating the systematic parallax offset of $-82 \pm 33 \mu$ as found by Stassun \& Torres (2018).

To correct for reddening we use the 3D dust map of Leike et al. (2020), implemented within the python package dustmaps (Green 2018). Targeting bright, cool dwarfs as we do here automatically means our stars will be relatively close, and we take those within the Local Bubble, two thirds of our sample, to be unreddened $(\lesssim 70 \mathrm{pc}$, e.g. Leroy 1993; Lallement et al. 2003) so long as the Gaia $G$ band extinction reported by the dust map is consistent with zero $\left(A_{G}<0.01\right)$. Nominal extinction coefficients were sourced from Casagrande \& VandenBerg (2014) for the 2MASS $J H K_{S}$ bands and Casagrande et al. (2019) for SkyMapper uvgriz, with Gaia $G, B_{P}$, and $R_{P}$ coefficients computed from the relation given in Casagrande et al. (2020) for $B_{P}-R_{P}=2.03$, the median value for our sample.

\subsection{Standard Selection}

Given the complexities involved in determining the properties of cool dwarfs, we also observed a set of 136 well characterised late $\mathrm{K} / \mathrm{M}$-dwarf standards from the literature. Broadly these standards have parameters from at least one of the following sources:

(i) $[\mathrm{Fe} / \mathrm{H}]$ from an FGK companion,

(ii) $[\mathrm{Fe} / \mathrm{H}]$ from low resolution NIR spectra,

(iii) $T_{\text {eff }}$ from interferometry.

With the exception of available interferometric $T_{\text {eff }}$ standards, we additionally wanted to source standards from large uniform catalogues due to the known problem of systematics between different spectroscopic techniques (e.g. Lebzelter et al. 2012; Hinkel et al. 2016). With this in mind, the bulk of our M/late-K dwarf standards

\footnotetext{
2 Expected to be approximately 1.0 in case where the single star model provides a good fit for the astrometric data. See: https://gea.esac. esa.int/archive/documentation/GDR2/Gaia_archive/chap_ datamodel/sec_dm_main_tables/ssec_dm_ruwe.html

3 Though we do accept TIC 158588995 with a marginal RUWE 1.47 as it sits on the main sequence and does not appear overluminous.

4 https://exofop.ipac.caltech.edu/tess/
}

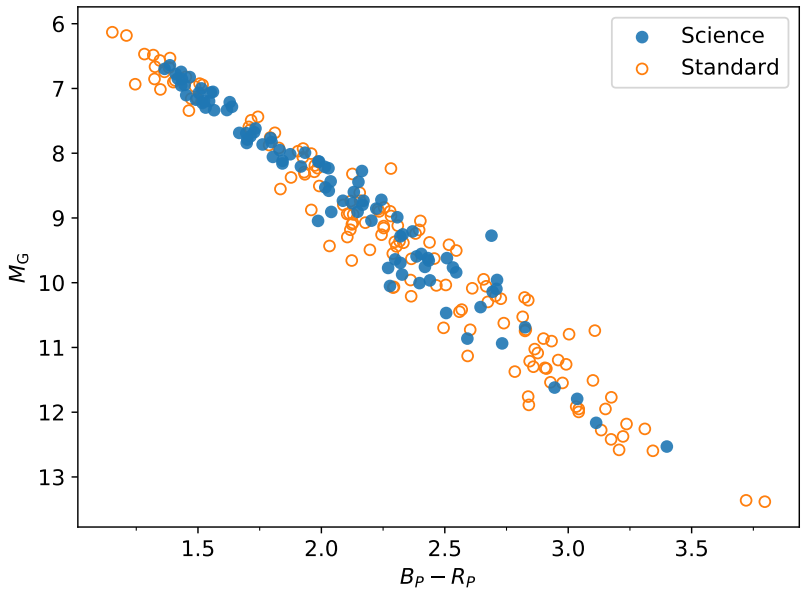

Figure 1. Gaia DR2 $M_{G}$ versus $\left(B_{p}-R_{p}\right)$ colour magnitude diagram for science targets (filled blue circles) and cool dwarf standards (orange open circles).

come from the works of Rojas-Ayala et al. (2012) and Mann et al. (2015), with interferometric targets from von Braun et al. (2012), Boyajian et al. (2012), von Braun et al. (2014), Rabus et al. (2019), and Rains et al. (2020); and FGK companion $[\mathrm{Fe} / \mathrm{H}]$ compiled by Newton et al. (2014) from Valenti \& Fischer (2005), Sousa et al. (2006) and Sozzetti et al. (2009). Our mid-K dwarf calibrators do not come from a single uniform catalogue; they are instead pulled from the works of Woolf \& Wallerstein (2005), Sousa et al. (2008), Prugniel et al. (2011), Sousa et al. (2011), Tsantaki et al. (2013), Luck (2017), Luck (2018) and Montes et al. (2018).

These stars were observed with the same instrument settings as our science targets (but at higher SNR), with the intent to provide checks against our analysis techniques for this notoriously complex set of stars.

\subsection{Spectroscopic Observations}

Observations were conducted using the WiFeS instrument (WideField Spectrograph, Dopita et al. 2007) on the ANU 2.3 m Telescope at Siding Spring Observatory, Australia between August 2019 and September 2020. WiFeS, a dual camera integral field spectrograph, is an effective stellar survey instrument due to its high throughput and broad wavelength coverage. Using the B3000 and R7000 gratings, and RT480 beam splitter, we obtain low resolution blue spectra (3500 $\leqslant \lambda \leqslant 5700 \AA, \lambda / \Delta \lambda \sim 3000)$ and moderate resolution red spectra $(5400 \leqslant \lambda \leqslant 7000 \AA, \lambda / \Delta \lambda \sim 7000)$ with median signal to noise ratios (SNR) per spectral pixel of 16 and 58 respectively. Exposure times ranged from $20 \mathrm{sec}$ to $30 \mathrm{~min}$, and were chosen on the basis of 0.5 magnitude bins in Gaia $G$.

Target observations were bracketed hourly with NeAr Arc lamp exposures, telluric standards were observed every few hours, and flux standards were observed several times throughout each night. Data reduction was done using the standard PyWiFeS pipeline (Childress et al. 2014) with the exception of custom flux calibration due to PyWiFeS' poor performance with R7000 spectra. Science target observations are listed in Table A1, and standard star observations in Table B1. 
Table 1. Science targets

\begin{tabular}{|c|c|c|c|c|c|c|c|c|c|c|c|}
\hline $\mathrm{TOI}^{a}$ & $\mathrm{TIC}^{b}$ & $2 \mathrm{MASS}^{c}$ & Gaia DR2 $^{d}$ & $\begin{array}{c}\mathrm{RA}^{d} \\
\text { (hh mm ss.ss) }\end{array}$ & $\begin{array}{c}\text { DEC }^{d} \\
\text { (dd mm ss.ss) }\end{array}$ & $\begin{array}{c}G^{d} \\
(\mathrm{mag})\end{array}$ & $\begin{array}{c}B_{p}-R_{p}{ }^{d} \\
(\mathrm{mag})\end{array}$ & $\begin{array}{l}\mathrm{Plx}^{d} \\
\text { (mas) }\end{array}$ & ruwe $^{d}$ & $\mathrm{E}(\boldsymbol{B}-\boldsymbol{V})$ & $\mathrm{N}_{\mathrm{pc}}^{e}$ \\
\hline 741 & 359271092 & 09213761-6016551 & 5299440441521812992 & 092135.86 & -614307.68 & 8.68 & 2.03 & $95.63 \pm 0.03$ & 1.1 & 0.00 & 1 \\
\hline 731 & 34068865 & 09442986-4546351 & 5412250540681250560 & 094429.16 & -461315.60 & 9.15 & 2.32 & $106.21 \pm 0.03$ & 1.1 & 0.00 & 1 \\
\hline 260 & 37749396 & 00190556-0957530 & 2428162410789155328 & 001905.52 & -100201.68 & 9.31 & 1.70 & $49.51 \pm 0.06$ & 0.9 & 0.00 & 1 \\
\hline 836 & 440887364 & $15001942-2427147$ & 6230733559097425152 & 150019.18 & -253244.88 & 9.39 & 1.55 & $36.33 \pm 0.04$ & 1.0 & 0.01 & 2 \\
\hline 562 & 413248763 & 09360161-2139371 & 5664814198431308288 & 093601.80 & -222005.64 & 9.88 & 2.40 & $105.88 \pm 0.06$ & 1.1 & 0.00 & 3 \\
\hline 455 & 98796344 & $03015142-1635356$ & 5153091836072107136 & 030151.00 & -172419.80 & 10.05 & 2.59 & $145.55 \pm 0.08$ & 1.1 & 0.00 & 2 \\
\hline 139 & 62483237 & $22253655-3454346$ & 6598814657249555328 & 222536.58 & -350525.08 & 10.08 & 1.45 & $23.55 \pm 0.04$ & 1.0 & 0.00 & 1 \\
\hline 253 & 322063810 & 00571629-5135048 & 4928367189956040960 & 005716.44 & -522452.92 & 10.18 & 1.71 & $32.39 \pm 0.03$ & 1.0 & 0.00 & 1 \\
\hline 134 & 234994474 & $23200751-6003545$ & 6491962296196145664 & 232006.86 & -615603.48 & 10.23 & 2.03 & $39.73 \pm 0.04$ & 1.0 & 0.00 & 1 \\
\hline 544 & 50618703 & 05290957-0020331 & 3220926542276901888 & 052909.62 & -13925.56 & 10.40 & 1.57 & $24.29 \pm 0.04$ & 1.1 & 0.01 & 1 \\
\hline 486 & 260708537 & 06334998-5831426 & 5482827676662168832 & 063349.18 & -592830.00 & 10.53 & 2.43 & $65.70 \pm 0.03$ & 1.2 & 0.00 & 1 \\
\hline 177 & 262530407 & $01214538-4642518$ & 4933912198893332224 & 012145.22 & -471707.08 & 10.55 & 2.17 & $44.46 \pm 0.05$ & 1.0 & 0.00 & 1 \\
\hline 129 & 201248411 & $00004490-5449498$ & 4923860051276772608 & 000044.54 & -551009.12 & 10.59 & 1.39 & $16.16 \pm 0.02$ & 1.1 & 0.00 & 1 \\
\hline 175 & 307210830 & 08180763-6818468 & 5271055243163629056 & 081807.90 & -694107.80 & 10.60 & 2.51 & $94.14 \pm 0.03$ & 1.1 & 0.00 & 3 \\
\hline 824 & 193641523 & $14483982-5735175$ & 5880886001621564928 & 144839.72 & -582439.96 & 10.72 & 1.36 & $15.61 \pm 0.03$ & 1.1 & 0.02 & 1 \\
\hline 133 & 219338557 & $23373497-5857166$ & 6489346046933733632 & 233735.38 & -590241.64 & 10.72 & 1.53 & $20.53 \pm 0.03$ & 1.0 & 0.00 & 1 \\
\hline 1130 & 254113311 & $19053021-4126151$ & 6715688452614516736 & 190530.24 & -423344.64 & 10.88 & 1.55 & $17.14 \pm 0.05$ & 1.1 & 0.01 & 2 \\
\hline 198 & 12421862 & $00090428-2707196$ & 2333676738049780352 & 000905.16 & -285241.88 & 10.92 & 1.99 & $42.12 \pm 0.05$ & 1.0 & 0.00 & 1 \\
\hline 833 & 362249359 & 09423526-6228346 & 5250780970316845696 & 094234.92 & -633126.76 & 11.05 & 1.83 & $23.94 \pm 0.02$ & 0.8 & 0.01 & 1 \\
\hline 178 & 251848941 & 00291228-3027133 & 2318295979126499200 & 002912.48 & -313245.24 & 11.15 & 1.49 & $15.92 \pm 0.05$ & 1.2 & 0.00 & 3 \\
\hline 279 & 122613513 & 02444524-3212391 & 5063070558501465856 & 024445.24 & -334720.40 & 11.20 & 1.44 & $13.42 \pm 0.04$ & 1.1 & 0.00 & 1 \\
\hline 704 & 260004324 & $06042035-5518468$ & 5500061456275483776 & 060421.60 & -564118.60 & 11.23 & 2.22 & $33.48 \pm 0.03$ & 1.0 & 0.00 & 1 \\
\hline 1078 & 370133522 & $20274210-5627262$ & 6468968316900356736 & 202742.86 & -573215.72 & 11.24 & 2.32 & $49.06 \pm 0.05$ & 1.0 & 0.00 & 1 \\
\hline 969 & 280437559 & $07403284+0205561$ & 3087206553745290624 & 074032.81 & +20554.96 & 11.25 & 1.47 & $12.92 \pm 0.05$ & 1.4 & 0.01 & 1 \\
\hline 620 & 296739893 & 09284158-1209551 & 5738284016370287616 & 092841.62 & -134958.08 & 11.31 & 2.24 & $30.25 \pm 0.05$ & 1.0 & 0.00 & 1 \\
\hline 910 & 369327947 & $19205439-8233170$ & 6347643496607835520 & 192057.10 & -832624.72 & 11.42 & 2.73 & $80.09 \pm 0.04$ & 1.1 & 0.00 & 1 \\
\hline 713 & 167600516 & $06480517-6537252$ & 5285060409961261696 & 064805.14 & -662232.52 & 11.42 & 1.52 & $14.39 \pm 0.02$ & 0.9 & 0.01 & 2 \\
\hline 932 & 260417932 & $06234590-5434414$ & 5499671713762981248 & 062345.82 & -552519.20 & 11.42 & 1.41 & $11.68 \pm 0.02$ & 0.8 & 0.01 & 2 \\
\hline 240 & 101948569 & 00590112-4409389 & 4982951791883929472 & 005901.18 & -455020.76 & 11.43 & 1.50 & $13.33 \pm 0.03$ & 1.1 & 0.00 & 1 \\
\hline 696 & 77156829 & 04324261-3947112 & 4864160624337973248 & 043242.96 & -401232.76 & 11.54 & 2.28 & $50.28 \pm 0.02$ & 1.1 & 0.00 & 2 \\
\hline 244 & 118327550 & 00421695-3643053 & 5001098681543159040 & 004216.75 & -371655.20 & 11.55 & 2.55 & $45.36 \pm 0.07$ & 1.3 & 0.00 & 1 \\
\hline 270 & 259377017 & 04333970-5157222 & 4781196115469953024 & 043339.86 & -520233.36 & 11.63 & 2.33 & $44.46 \pm 0.03$ & 1.0 & 0.00 & 3 \\
\hline 912 & 406941612 & $15172165-8028225$ & 5772442647192375808 & 151718.86 & -813136.12 & 11.64 & 2.40 & $38.27 \pm 0.02$ & 1.1 & 0.01 & 1 \\
\hline 475 & 100608026 & 05465951-3231592 & 2901089987127041920 & 054659.59 & -332803.00 & 11.70 & 1.76 & $16.99 \pm 0.02$ & 1.0 & 0.01 & 1 \\
\hline 442 & 70899085 & $04164560-1205023$ & 3189306030970782208 & 041645.65 & -135454.36 & 11.73 & 1.99 & $18.98 \pm 0.04$ & 1.2 & 0.00 & 1 \\
\hline 761 & 165317334 & $11570326-3806169$ & 3460168250866990848 & 115703.12 & -395342.72 & 11.73 & 1.73 & $14.94 \pm 0.04$ & 1.2 & 0.01 & 1 \\
\hline 870 & 219229644 & $04131645-5056400$ & 4782093729275660160 & 041316.63 & -510320.52 & 11.78 & 1.99 & $18.56 \pm 0.02$ & 1.1 & 0.00 & 1 \\
\hline 904 & 261257684 & 05572938-8307486 & 4620844400530949376 & 055729.11 & -845213.08 & 11.84 & 2.02 & $21.67 \pm 0.02$ & 1.0 & 0.01 & 1 \\
\hline 732 & 36724087 & 10183516-1142599 & 3767281845873242112 & 101834.78 & -121655.92 & 11.85 & 2.69 & $45.46 \pm 0.08$ & 1.1 & 0.00 & 2 \\
\hline 656 & 36734222 & $10193800-0948225$ & 3767805209112436736 & 101937.97 & -101136.96 & 11.89 & 1.63 & $11.50 \pm 0.04$ & 1.1 & 0.01 & 1 \\
\hline 1075 & 351601843 & 20395334-6526579 & 6426188308031756288 & 203953.09 & -663301.08 & 12.05 & 1.84 & $16.24 \pm 0.02$ & 1.2 & 0.00 & 1 \\
\hline 700 & 150428135 & $06282325-6534456$ & 5284517766615492736 & 062822.97 & -662517.04 & 12.06 & 2.39 & $32.10 \pm 0.02$ & 1.1 & 0.00 & 3 \\
\hline 727 & 149788158 & 08425684-0229529 & 3072157538091829120 & 084256.86 & -33005.04 & 12.07 & 2.15 & $23.24 \pm 0.03$ & 1.1 & 0.00 & 1 \\
\hline 249 & 179985715 & 00561930-3856552 & 4987729474846997248 & 005619.20 & -390302.88 & 12.08 & 1.70 & $14.13 \pm 0.03$ & 1.1 & 0.00 & 1 \\
\hline 1201 & 29960110 & 02485926-1432152 & 5157183324996790272 & 024859.45 & -152745.72 & 12.10 & 2.37 & $26.37 \pm 0.04$ & 1.1 & 0.00 & 1 \\
\hline
\end{tabular}

Notes: ${ }^{a}$ TESS Object of Interest ID, ${ }^{b}$ TESS Input Catalogue ID (Stassun et al. 2018, 2019), ${ }^{c}$ 2MASS (Skrutskie et al. 2006), ${ }^{c}$ Gaia (Brown et al. 2018) - note that Gaia parallaxes listed here have been corrected for the zeropoint offset, ${ }^{d}$ Number of candidate planets, NASA Exoplanet Follow-up Observing Program for TESS 
Table 1 - continued Science targets

\begin{tabular}{|c|c|c|c|c|c|c|c|c|c|c|c|}
\hline $\mathrm{TOI}^{a}$ & $\mathrm{TIC}^{b}$ & 2 MASS $^{c}$ & Gaia DR2 $^{d}$ & $\begin{array}{c}\mathrm{RA}^{d} \\
\text { (hh mm ss.ss) }\end{array}$ & $\begin{array}{c}\mathrm{DEC}^{d} \\
\text { (dd } \mathrm{mm} \text { ss.ss) }\end{array}$ & $\begin{array}{c}G^{d} \\
(\mathrm{mag})\end{array}$ & $\begin{array}{c}B_{p}-R_{p}{ }^{d} \\
(\mathrm{mag})\end{array}$ & $\begin{array}{l}\mathrm{Plx}^{d} \\
\text { (mas) }\end{array}$ & ruwe $^{d}$ & $\mathrm{E}(B-V)$ & $\mathrm{N}_{\mathrm{pc}}^{e}$ \\
\hline 875 & 14165625 & $05120890-3742313$ & 4820828591913853696 & 051208.93 & -381729.40 & 12.12 & 1.51 & $9.39 \pm 0.02$ & 1.0 & 0.01 & 1 \\
\hline 929 & 175532955 & 03033741-3955515 & 5044287532642519680 & 030337.73 & -400409.12 & 12.13 & 1.42 & $8.71 \pm 0.02$ & 1.1 & 0.01 & 1 \\
\hline 493 & 19025965 & $07583071+1253005$ & 3151371883379694720 & 075830.65 & +125259.88 & 12.19 & 1.56 & $9.29 \pm 0.04$ & 1.1 & 0.01 & 1 \\
\hline 1216 & 141527965 & 05505139-7541200 & 4648441970589471104 & 055051.55 & -761841.04 & 12.31 & 1.62 & $10.02 \pm 0.03$ & 1.2 & 0.01 & 1 \\
\hline 233 & 415969908 & $22545039-1854426$ & 2402715141877299584 & 225450.06 & -190515.36 & 12.41 & 2.27 & $29.58 \pm 0.04$ & 1.0 & 0.00 & 2 \\
\hline 711 & 38510224 & $04100386-6156326$ & 4676789514954240768 & 041003.86 & -620326.28 & 12.45 & 1.45 & $8.43 \pm 0.02$ & 1.0 & 0.01 & 1 \\
\hline 876 & 32497972 & 05362611-2414377 & 2963392606627366912 & 053626.23 & -254520.16 & 12.47 & 1.87 & $12.76 \pm 0.03$ & 1.1 & 0.01 & 1 \\
\hline 785 & 374829238 & 05532099-6538022 & 4755884700667639296 & 055320.95 & -662159.40 & 12.51 & 2.04 & $15.23 \pm 0.02$ & 1.0 & 0.01 & 1 \\
\hline 406 & 153065527 & $03170297-4214323$ & 4851053999056603904 & 031703.02 & -434521.24 & 12.55 & 2.71 & $32.17 \pm 0.04$ & 1.2 & 0.00 & 2 \\
\hline 714 & 219195044 & 06093401-5349245 & 5500474185452572032 & 060934.18 & -541037.20 & 12.56 & 2.04 & $18.54 \pm 0.02$ & 1.2 & 0.01 & 2 \\
\hline 900 & 210873792 & $16233735-3122228$ & 6037266684232926208 & 162337.22 & -323735.04 & 12.59 & 1.52 & $8.23 \pm 0.05$ & 0.9 & 0.03 & 1 \\
\hline 557 & 55488511 & 03560411-1016192 & 3193508849745633280 & 035604.27 & -114340.80 & 12.60 & 1.92 & $13.14 \pm 0.04$ & 1.1 & 0.01 & 1 \\
\hline 864 & 231728511 & $05254662-5121253$ & 4772266186971169792 & 052546.42 & -523834.80 & 12.66 & 2.42 & $26.22 \pm 0.03$ & 1.1 & 0.01 & 1 \\
\hline 256 & 92226327 & 00445930-1516166 & 2371032916186181760 & 004459.66 & -164333.24 & 12.67 & 3.04 & $66.70 \pm 0.07$ & 1.1 & 0.00 & 2 \\
\hline 702 & 237914496 & 03444203-6511567 & 4672700190692201088 & 034441.98 & -664806.12 & 12.68 & 1.80 & $11.82 \pm 0.03$ & 1.1 & 0.01 & 1 \\
\hline 1082 & 261108236 & $05330624-8048563$ & 4621526273835900288 & 053306.19 & -811104.20 & 12.68 & 1.70 & $9.95 \pm 0.03$ & 1.1 & 0.01 & 1 \\
\hline 672 & 151825527 & 11115769-3919400 & 5396580575830873728 & 111157.82 & -404018.84 & 12.72 & 2.13 & $14.92 \pm 0.03$ & 1.1 & 0.01 & 1 \\
\hline 806 & 33831980 & 04134003-7605515 & 4627952094666051072 & 041339.86 & -775407.20 & 12.77 & 1.67 & $9.55 \pm 0.02$ & 1.3 & 0.01 & 1 \\
\hline 797 & 271596225 & $07141480-7436089$ & 5262540590756812032 & 071415.14 & -752348.84 & 12.78 & 2.20 & $17.77 \pm 0.02$ & 1.1 & 0.01 & 2 \\
\hline 663 & 54962195 & $10401596-0830385$ & 3762515188088861184 & 104015.82 & -92920.04 & 12.81 & 2.13 & $15.54 \pm 0.04$ & 1.0 & 0.01 & 2 \\
\hline 540 & 200322593 & $05051443-4756154$ & 4785886941312921344 & 050514.33 & -480345.00 & 12.89 & 3.11 & $71.39 \pm 0.04$ & 1.0 & 0.00 & 1 \\
\hline 285 & 220459976 & 04584731-5623385 & 4764216563561182336 & 045847.33 & -573622.32 & 13.07 & 1.79 & $8.61 \pm 0.02$ & 0.9 & 0.01 & 1 \\
\hline 674 & 158588995 & 10582099-3651292 & 5400949450924312576 & 105820.78 & -370830.84 & 13.08 & 2.53 & $21.67 \pm 0.05$ & 1.5 & 0.01 & 1 \\
\hline 789 & 300710077 & 07410444-7118157 & 5264306681309492864 & 074104.85 & -724146.32 & 13.15 & 2.44 & $23.01 \pm 0.03$ & 1.3 & 0.01 & 1 \\
\hline 873 & 237920046 & $03465622-6320142$ & 4673392195823039744 & 034656.78 & -643947.16 & 13.16 & 2.09 & $12.97 \pm 0.02$ & 1.3 & 0.01 & 1 \\
\hline 698 & 141527579 & 05505661-7637132 & 4647922867959139072 & 055057.38 & -772249.44 & 13.26 & 2.33 & $15.75 \pm 0.03$ & 1.2 & 0.01 & 1 \\
\hline 136 & 410153553 & 22415815-6910089 & 6385548541499112448 & 224159.09 & -704940.44 & 13.39 & 3.40 & $67.15 \pm 0.05$ & 1.1 & 0.00 & 1 \\
\hline 269 & 220479565 & 05032306-5410378 & 4770828304936109056 & 050323.11 & -554920.28 & 13.41 & 2.30 & $17.51 \pm 0.02$ & 1.1 & 0.01 & 1 \\
\hline 654 & 35009898 & 10585379-0532468 & 3788670679927991296 & 105853.90 & -62709.00 & 13.42 & 2.51 & $17.29 \pm 0.05$ & 1.1 & 0.01 & 1 \\
\hline 782 & 429358906 & $12154108-1854365$ & 3518374197418907648 & 121540.90 & -190522.92 & 13.55 & 2.71 & $19.01 \pm 0.07$ & 1.2 & 0.01 & 1 \\
\hline 521 & 27649847 & $08132251+1213181$ & 649852779797683968 & 081322.63 & +121319.56 & 13.58 & 2.44 & $16.38 \pm 0.06$ & 1.3 & 0.00 & 1 \\
\hline 203 & 259962054 & 02520450-6741155 & 4647534190597951232 & 025204.34 & -681846.80 & 13.59 & 2.94 & $40.31 \pm 0.05$ & 1.4 & 0.00 & 1 \\
\hline 532 & 144700903 & $05401918+1133463$ & 3340265717587057536 & 054019.22 & +113345.36 & 13.63 & 1.93 & $7.38 \pm 0.03$ & 1.1 & 0.02 & 1 \\
\hline 756 & 73649615 & $12482523-4528140$ & 6129327525817451648 & 124824.89 & -463146.20 & 13.66 & 2.31 & $11.58 \pm 0.04$ & 1.2 & 0.02 & 1 \\
\hline 302 & 229111835 & 01095538-5214219 & 4927215760764862976 & 010955.51 & -534537.80 & 13.71 & 1.64 & $5.10 \pm 0.01$ & 1.0 & 0.01 & 1 \\
\hline 435 & 44647437 & 03573850-2511238 & 5082797618168232320 & 035738.54 & -264836.36 & 13.75 & 1.73 & $6.02 \pm 0.02$ & 1.1 & 0.01 & 1 \\
\hline 1067 & 201642601 & $19144126-5934458$ & 6638412919991750912 & 191441.28 & -602514.16 & 13.82 & 1.43 & $3.76 \pm 0.02$ & 1.0 & 0.03 & 1 \\
\hline 210 & 141608198 & 05555049-7359046 & 4650160717726370816 & 055550.83 & -740052.92 & 13.84 & 2.83 & $23.33 \pm 0.05$ & 1.3 & 0.01 & 1 \\
\hline 1073 & 158297421 & $19095625-4939538$ & 6658373007402886400 & 190956.26 & -502006.36 & 14.31 & 1.43 & $3.30 \pm 0.04$ & 1.0 & 0.04 & 1 \\
\hline 468 & 33521996 & 05523523-1901539 & 2966680597368750720 & 055235.23 & -205806.24 & 14.34 & 2.01 & $5.88 \pm 0.04$ & 1.2 & 0.01 & 1 \\
\hline 122 & 231702397 & $22114728-5856422$ & 6411096106487783296 & 221147.57 & -590314.04 & 14.34 & 2.64 & $16.07 \pm 0.06$ & 1.2 & 0.00 & 1 \\
\hline 507 & 348538431 & 08063103-1545526 & 5724250571514167424 & 080631.10 & -161407.08 & 14.48 & 2.69 & $8.99 \pm 0.06$ & 1.3 & 0.01 & 1 \\
\hline 551 & 192826603 & 05305145-3637508 & 4821739369794767744 & 053051.41 & -372208.40 & 14.83 & 1.84 & $4.56 \pm 0.02$ & 1.0 & 0.02 & 1 \\
\hline 552 & 44737596 & $04034783-2524320$ & 5082914338199586560 & 040347.86 & -263527.96 & 14.87 & 2.15 & $5.10 \pm 0.03$ & 1.1 & 0.01 & 1 \\
\hline 234 & 12423815 & 00101648-2616566 & 2335244779070099200 & 001016.54 & -274303.36 & 15.69 & 2.17 & $3.98 \pm 0.07$ & 1.1 & 0.01 & 1 \\
\hline 555 & 170849515 & 04412154-3219128 & 4877426575724467456 & 044121.55 & -334046.56 & 15.71 & 1.80 & $2.56 \pm 0.04$ & 1.0 & 0.04 & 1 \\
\hline 142 & 425934411 & $00182539-6250523$ & 4901321849613348736 & 001825.42 & -630907.56 & 15.77 & 2.16 & $3.09 \pm 0.04$ & 1.0 & 0.01 & 1 \\
\hline
\end{tabular}




\subsection{Radial velocity determination}

Radial velocities of the WiFeS R7000 spectra were determined from a least squares minimisation of a set of synthetic template spectra varying in temperature (see Section 4.1 for details of model grid). We use a coarsely sampled version of this grid, computed at $\mathrm{R} \sim 7000$ over $5400 \leqslant \lambda \leqslant 7000$ for $3000 \leqslant T_{\text {eff }} \leqslant 5500 \mathrm{~K}$, $\log g=4.5$, and $[\mathrm{Fe} / \mathrm{H}]=0.0$, with $T_{\text {eff }}$ steps of $100 \mathrm{~K}$ for radial velocity determination. For further information on our RV fitting formalism, see Žerjal et al. $(2021)^{5}$.

Statistical uncertainties on this approach are median $\sim 410 \mathrm{~m} \mathrm{~s}^{-1}$, though comparison to Gaia DR2 in Figure 2 reveals a larger scatter with standard deviation $\sim 4.5 \mathrm{~km} \mathrm{~s}^{-1}$, computed from a median absolute deviation, which we add in quadrature with our statistical uncertainties. Higher uncertainties are consistent with the work of Kuruwita et al. (2018) who found that WiFeS varies on shorter timescales than our hourly arcs can account for. While they additionally improved precision by calibrating using oxygen B-band absorption, RV uncertainties of $\sim 4.5 \mathrm{~km} \mathrm{~s}^{-1}$ are sufficient for this work. Our final values are reported in Table A1 for science targets, and Table B1 for standards.

\section{PHOTOMETRIC METALLICITY CALIBRATION}

As established earlier, cool dwarf metallicities are notoriously difficult to determine, particularly when working with optical spectra. Bonfils et al. (2005) initially proposed empirical calibrations to determine $[\mathrm{Fe} / \mathrm{H}]$ from a star's position in $M_{K}-(V-K)$ space, a technique which was later iterated on by Johnson \& Apps (2009), Schlaufman \& Laughlin (2010), and Neves et al. (2012). Such relations are based on the fact that once on the main sequence, low mass stars do not evolve (and hence change in brightness and temperature) appreciably on moderate timescales as compared to their higher mass and faster evolving counterparts. Thus, assuming no extra scatter from unresolved binaries and standard helium enrichment (e.g. Pagel \& Portinari 1998), a star's position above or below the mean main sequence is directly correlated with its chemical composition (Baraffe et al. 1998).

These relations are benchmarked on what is considered the gold standard for M-dwarf metallicites: $[\mathrm{Fe} / \mathrm{H}]$ from a hotter FGK companion taken to have formed at the same time and thus have the same chemical composition. This chemical homogeneity is now well established for FGK-FGK pairs (e.g. Desidera et al. 2004; Hawkins et al. 2020). The process of determining which stars on the sky are likely associated has now been greatly simplified with the release of Gaia DR2, which has provided precision parallax measurements and proper motions for nearly all nearby M-dwarfs, with our sample of secondaries having median $0.17 \%$ parallax precision.

We take as input the sample of FGK-KM-dwarf pairs compiled by Mann et al. (2013a) and Newton et al. (2014). These combine primary star $[\mathrm{Fe} / \mathrm{H}]$ measurements from high resolution spectra sourced from a variety of previous surveys (Mishenina et al. 2004; Luck \& Heiter 2005; Valenti \& Fischer 2005; Bean et al. 2006a; Ramírez et al. 2007; Robinson et al. 2007; Fuhrmann 2008; Casagrande et al. 2011; da Silva et al. 2011; Mann et al. 2013a), with Mann et al. (2013a) correcting for inter-survey systematics to place them on a common $[\mathrm{Fe} / \mathrm{H}]$ scale. To this set we add the

5 Our RV fitting code, along with all other code for this project, can be found at https://github.com/adrains/plumage

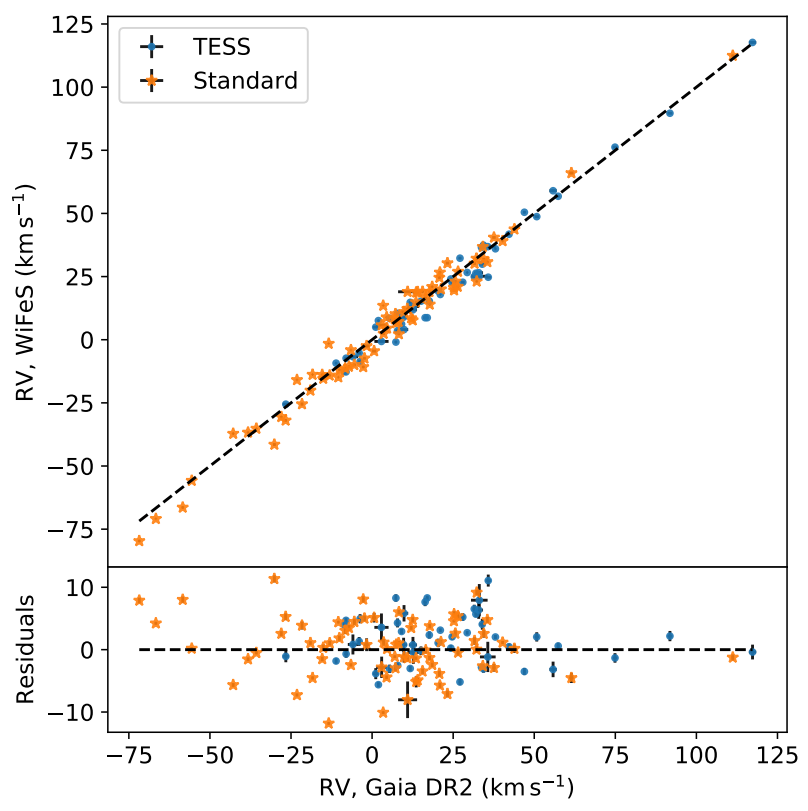

Figure 2. Comparison between those stars with radial velocities in Gaia DR2 and our work here, from which we determine a scatter of $\sim 4.5 \mathrm{~km} \mathrm{~s}^{-1}$.

metal-poor, cool subdwarf VB12 to extend our metallicity coverage, taking the $[\mathrm{Fe} / \mathrm{H}]$ reported by Ramírez et al. (2007) for its primary HD $219617 \mathrm{AB}$ (and correcting for the systematic reported by Mann et al. 2013a). This provided 128 total pairs, which was reduced to 69 after crossmatching with both Gaia DR2 and 2MASS, and removing those stars with missing or poor photometry (2MASS Qflg $\neq$ 'AAA', where 'AAA' is the highest photometric quality rating and corresponds to $J H K_{S}$ respectively); those flagged on SIMBAD ${ }^{6}$ as spectroscopic binaries; those with poor Gaia astrometry (Gaia dup flag=1, RUWE > 1.4); those pairs with M dwarf primaries; or whose parallaxes, astrometry, and RVs indicate they aren't associated with the putative primary. These 69 stars are listed in Table C1, and span $-1.28<[\mathrm{Fe} / \mathrm{H}]<+0.56$.

From this sample we follow the approaches of Johnson \& Apps (2009) and Schlaufman \& Laughlin (2010) and use a polynomial to trace the mean main sequence in $M_{K_{S}}$-colour space, though using $\left(B_{P}-K_{S}\right)$ instead of $\left(V-K_{S}\right)$. For our main sequence fit, we use the complete Mann et al. (2015) sample of cool dwarfs with Gaia parallaxes, which spans a wider range in $\left(B_{P}-K_{S}\right)$ and is less sparse than the assembled sample of M-dwarf secondaries. We find the following third order polynomial sufficient to describe the main sequence:

$\left(B_{P}-K_{S}\right)=a_{3} M_{K_{S}}^{3}+a_{2} M_{K_{S}}^{2}+a_{1} M_{K_{S}}+a_{0}$

where $a_{3}=0.05385, a_{2}=-1.08356, a_{1}=7.76175$, and $a_{0}=-14.54705$. We then calculate the offset in $\left(B_{P}-K_{S}\right)$ from this polynomial (as a colour offers greater discriminatory power than $M_{K_{S}}$, Schlaufman \& Laughlin 2010), and use least squares to find the best fitting linear relation for $[\mathrm{Fe} / \mathrm{H}]$ :

$[\mathrm{Fe} / \mathrm{H}]=b_{1} \Delta\left(B_{P}-K_{S}\right)+b_{0}$

where $b_{1}$ and $b_{0}$ are the linear polynomial coefficients. After cor-

6 http://simbad.u-strasbg.fr/simbad/ 
recting for a remaining trend in the residuals, our adopted coefficients are $b_{1}=0.71339$, and $b_{0}=-0.04301$. This relation is valid for stars with $1.51<\left(B_{P}-R_{P}\right)<3.3$ (based on the hottest and coolest secondaries respectively), and has an uncertainty of \pm 0.19 dex (from the standard deviation in the residuals). We stress that the relation should only be used for stars that pass the same quality cuts we use to build the relation: unsaturated photometry, not flagged as a duplicate source in Gaia, RUWE $<1.4$, and not a known/suspected spectroscopic binary or pre-main sequence star. Our $[\mathrm{Fe} / \mathrm{H}]$ recovery and fits can be seen in Figure 3.

\section{SPECTROSCOPIC ANALYSIS}

The TESS candidate planet host observing program described here developed from an ANU $2.3 \mathrm{~m} / \mathrm{WiFeS}$ survey of potential young stars (Žerjal et al. 2021) to identify signs of youth (via Balmer Series and Ca II H\&K emission, and Li $6708 \AA$ absorption) and determine RVs to enable kinematic analysis with Chronostar (Crundall et al. 2019) when combined with Gaia astrometry. While their spectral type coverage $\left(1.27<B_{P}<2.6\right)$ was relatively similar to our own, instrument setup however prioritised higher spectral resolution for improved velocity precision and coverage of the key wavelength regions of interest. These regions are firmly in the optical, where Mdwarf spectral features are strongly blended and heavily dominated by molecular absorption from hydrides (e.g. $\mathrm{MgH}, \mathrm{CaH}, \mathrm{SiH}$ ) and oxides (e.g. TiO, $\mathrm{VO}, \mathrm{ZrO}$ ). This is in contrast to most of the previous low-medium resolution studies of M-dwarfs which work in the NIR where the absorption is less severe and many more $[\mathrm{Fe} / \mathrm{H}]$ sensitive features are available.

Here we describe our attempts to derive reliable atmospheric parameters from our spectra using a model based approach. Our investigation ultimately revealed substantial systematics and degeneracies when fitting to model optical spectra, resulting in our inability to recover $\log g$ or $[\mathrm{Fe} / \mathrm{H}]$. While the spectra are included in our temperature fitting routine, they are primarily used for RV determination, identification of peculiarities (such as signs of youth), and for testing model fluxes. The details of our findings are covered below, and we await follow-up work to explore a standard-based or data-driven approach (e.g. similar to the work of Birky et al. 2020, but in the optical) to take full advantage of the information in our now large library of optical cool dwarf spectra.

\subsection{Selection of Model Atmosphere Grid}

While synthetic spectra show better agreement for FGK stars, the onset of strong molecular features such as $\mathrm{TiO}$ and $\mathrm{H}_{2} \mathrm{O}$ in the atmospheres of late $\mathrm{K}$ and $\mathrm{M}$ dwarf atmospheres make the task of modelling their spectra far more complex. There are known historical issues, for instance, when computing optical colours from synthetic spectra (e.g. difficulties in computing accurate $V$ band magnitudes, Leggett et al. 1996), and the line lists required are considerably more complicated. Thus, before using models in our automatic fitting routine, we first investigate their performance at different wavelengths to flag regions requiring special consideration. For the purposes of this comparison, we check the MARCS grid of stellar atmospheres against the BT-Settl grid (Allard et al. 2011), both of which are described in detail below.

Our template grid of 1D LTE MARCS spectra was previously described by Nordlander et al. (2019) and computed using the TURBOSPECTRUM code (v15.1; Alvarez \& Plez 1998; Plez 2012) and MARCS model atmospheres (Gustafsson et al. 2008). The spectra are computed with a sampling resolution of $1 \mathrm{~km} \mathrm{~s}^{-1}$, corresponding to a resolving power of $R \sim 300000$, with a microturbulent velocity of $1 \mathrm{~km} \mathrm{~s}^{-1}$. We adopt the solar chemical composition and isotopic ratios from Asplund et al. (2009), except for an alpha enhancement that varies linearly from $[\alpha / \mathrm{Fe}]=0$ when $[\mathrm{Fe} / \mathrm{H}] \geqslant 0$ to $[\alpha / \mathrm{Fe}]=+0.4$ when $[\mathrm{Fe} / \mathrm{H}] \leqslant-1$. We use a selection of atomic lines from VALD3 (Ryabchikova et al. 2015) together with roughly 15 million molecular lines representing 18 different molecules, the most important of which for this work are $\mathrm{CaH}$ (Plez, priv. comm.), MgH (Kurucz 1995; Skory et al. 2003), and TiO (Plez 1998, with updates via VALD3).

MARCS model fluxes were developed for usage over a range of spectral types including both cool giants and, critically for our work here, cool dwarfs. Recent work fitting cool dwarf stellar atmospheres however have mostly used high-resolution NIR spectra (J band: Önehag et al. 2012; Lindgren et al. 2016; Lindgren \& Heiter 2017; $\mathrm{H}$ band: Souto et al. 2017, 2018) rather than the medium resolution optical spectra we use here.

For BT-Settl, we use the most recently published grid (Allard et al. 2012a,b; Baraffe et al. 2015) ${ }^{7}$ which uses abundances from Caffau et al. (2011) and covers $1200<T_{\text {eff }}<7000 \mathrm{~K}, 2.5<\log g<$ $5.5,[\mathrm{M} / \mathrm{H}]=0.0$. Note that while older grids have a wider range of $[\mathrm{M} / \mathrm{H}]$, they are also less complete in terms of physics and line lists, so we opt for the newest grid for our comparison here, and limit ourselves to testing on stars with approximately Solar $[\mathrm{Fe} / \mathrm{H}]$.

BT-Settl atmospheres have been developed with a focus on cool dwarf atmospheres and have a strong history of use for studying cool dwarfs at a variety of wavelengths and resolutions (e.g. Rojas-Ayala et al. 2012; Muirhead et al. 2012; Mann et al. 2012; Rajpurohit et al. 2013; Lépine et al. 2013; Gaidos et al. 2014; Mann et al. 2013c, 2015; Veyette et al. 2016, 2017; Souto et al. 2018). Most noteworthy for our comparison are tests by Reylé et al. (2011) and Mann et al. (2013c), which examined model performance at optical wavelength regions $>5500 \AA \AA$ common to our WiFeS R7000 spectra.

For each of our standard stars we combined and normalised our flux calibrated B3000 and R7000 spectra to give a single spectrum with $3500<\lambda<7000 \AA$. To this we compared synthetic MARCS fluxes interpolated to literature values of $T_{\mathrm{eff}}, \log g$, and $[\mathrm{Fe} / \mathrm{H}]$, as well as the BT-Settl equivalent for those with close to Solar $[\mathrm{Fe} / \mathrm{H}]$. Given our large library of standards we were able to observe model performance as a function of both stellar parameters and wavelength. A representative comparison (with overplotted filter bandpasses) is shown in Figure 4, and our main conclusions are summarised as follows:

- Both MARCS and BT-Settl models severely overpredict (worsening with decreasing $T_{\text {eff }}$ ) flux blueward of $\sim 5400 \AA$. The MARCS systematic offset is also a strong function of $[\mathrm{Fe} / \mathrm{H}]$, an effect also observed in Joyce \& Chaboyer (2015), and while this is likely also true for BT-Settl, we cannot comment definitively while limited to the Solar $[\mathrm{Fe} / \mathrm{H}]$ grid.

- BT-Settl additionally underpredicts flux at $\sim 6500 \AA$ (as expected from Reylé et al. 2011 and Mann et al. 2013c).

- Synthetic photometry generated in SkyMapper $v, g, r$, and Gaia $B_{P}$ is thus systematically brighter than the observed equivalents for reasonable assumptions of $T_{\mathrm{eff}}, \log g$, and $[\mathrm{Fe} / \mathrm{H}]$ for the star under consideration.

We are able to quantify these systematics by integrating pho-

\footnotetext{
7 https://phoenix.ens-lyon.fr/Grids/BT-Settl/CIFIST2011_ 2015/
} 

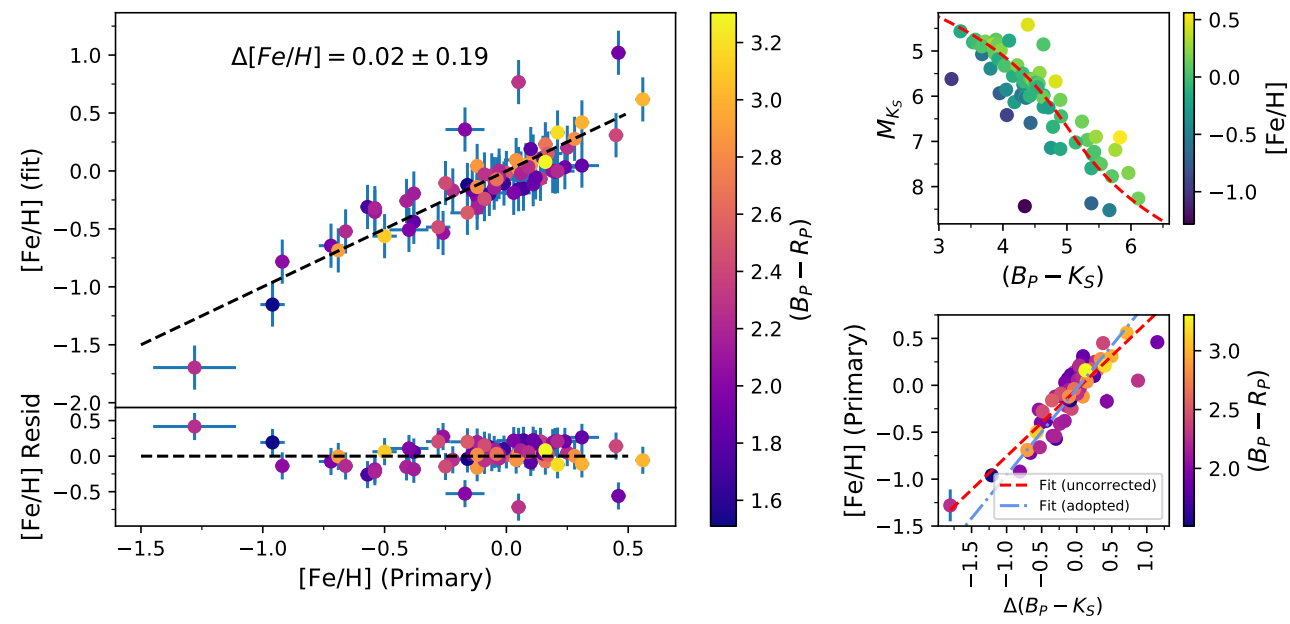

Figure 3. Left: Cool dwarf secondary $[\mathrm{Fe} / \mathrm{H}]$ calculated from our photometric calibration vs $[\mathrm{Fe} / \mathrm{H}]$ from the associated FGK primary star, colour coded by Gaia $\left(B_{P}-R_{P}\right)$. The standard deviation of the residuals, and our adopted uncertainty for the relation, is \pm 0.19 dex. See Table $C 1$ for further information on this FGK-KM binary calibration sample. Top Right: $M_{K_{S}}-\left(B_{P}-K_{S}\right)$ colour magnitude diagram for the calibration sample of cool dwarf secondaries colour coded by host star $[\mathrm{Fe} / \mathrm{H}]$. The dashed red line is a third order polynomial representing the main sequence, fitted to the Mann et al. (2015) sample of cool dwarfs. Bottom Right: Fitted $[\mathrm{Fe} / \mathrm{H}]$ as a function $\Delta\left(\boldsymbol{B}_{P}-K_{S}\right)$ offset from the mean main sequence polynomial. The dashed red line is the initial uncorrected linear least squares fit, and the dash-dotted blue line is the adopted fit after correcting for the remaining trend in the residuals

tometry from our flux calibrated observed spectra and comparing to the MARCS synthetic equivalents generated at the literature parameters for each star. Our wavelength coverage allows us to check the magnitude offsets $\Delta v, \Delta g, \Delta r$ and $\Delta B_{P}$, corresponding to $v, g$, $r$, and $B_{P}$ respectively. We note that for the purpose of this comparison we do not account for inaccuracies in our flux calibration, telluric absorption, nor for WiFeS not covering the bluest $\sim 200 \AA$ of $B_{P}$. However, checks with synthetic spectra show that this region accounts for less than $0.25 \%$ of $B_{P}$ flux at $3000 \mathrm{~K}$ where our correction is greatest, and remains less than $0.5 \%$ of flux at $4,500 \mathrm{~K}$ where our correction is more modest. These offsets are shown for $g, r$, and $B_{P}$ in Figure 5, and fit separately for each filter by the following linear relation in observed Gaia DR2 $\left(B_{P}-R_{P}\right)$ :

$\Delta m_{\zeta}=a_{1}\left(B_{P}-R_{P}\right)+a_{0}$

where $\Delta m_{\zeta}$ is the magnitude offset in filter $\zeta ; a_{1}$ equals $0.116,0.084$, and 0.034 for $g, r$, and $B_{P}$ fits respectively; and $a_{0}$ equals -0.072 , -0.069 , and -0.037 for $g, r$, and $B_{P}$ fits respectively. Computing the standard deviation for the residuals shows $0.10,0.05$, and 0.02 uncertainties in magnitude (equivalent to roughly $10 \%, 5 \%$, and $2 \%$ uncertainties in flux) for $g, r$, and $B_{P}$ respectively. From this we conclude that while the corrections to $r$, and $B_{P}$ are modest, $g$ is likely too affected to prove useful.

Following this both qualitative and quantitative investigation comparing model fluxes to our library of standard star spectra, we make the following decisions for our synthetic fitting methodology:

- Given similar observed systematics for both MARCS and BTSettl model fluxes, we adopt the MARCS grid to enable fitting for $[\mathrm{Fe} / \mathrm{H}]$ as well as $T_{\text {eff }}$ and $\log g$.

- Only use our R7000 spectra $(5400 \leqslant \lambda \leqslant 7000 \AA$ A ) for fitting, additionally masking out the two regions worst affected by missing opacities (5498-5585 $\AA$ and 6029-6159 $\AA$ ).

- Apply an observed $\left(B_{P}-R_{P}\right)$ dependent systematic offset to our generated synthetic $B_{P}$ and $r$ photometry per Equation 3 .

- Given the widespread historical use and success of studying
M-dwarfs at NIR wavelengths, we use $R_{P}, i, z, J, H$, and $K_{S}$ photometry assuming no substantial model systematics.

- However, to account for remaining model uncertainties, we add conservative \pm 0.011 magnitude ( $1 \%$ in flux) uncertainties in quadrature with the observed uncertainties for $R_{P}, i, z$; and the fitted \pm 0.02 for $r$, and \pm 0.05 for $B_{P}$.

\subsection{Synthetic Fitting}

Our approach to spectral fitting was developed specifically to work with the complicated spectra of our cool star sample and incorporates nine distinct sources of information. While it was hoped that this methodology would be sufficient to disentangle the strong degeneracy between $T_{\text {eff }}$ and $[\mathrm{Fe} / \mathrm{H}]$ and accurately recover distantindependent $[\mathrm{Fe} / \mathrm{H}]$ for our standard sample, this ultimately proved not to be the case. While we are able to tightly constrain $T_{\text {eff }}$, we must resort to using the photometric $[\mathrm{Fe} / \mathrm{H}]$ relation developed in Section 3 to fix $[\mathrm{Fe} / \mathrm{H}]$ during the fit. The information included in our fit is as follows:

(i) Medium resolution R7000 optical spectra from WiFeS,

(ii) Observed Gaia $B_{P}, R_{P} ; 2 \mathrm{MASS} J, H$, and $K_{S}$; and SkyMapper DR3 $r, i, z$ photometry,

(iii) Empirical cool dwarf radius relations from Mann et al. (2015) - valid for K7-M7 stars, and used to estimate $\log g$,

(iv) Empirical cool dwarf mass relations from Mann et al. (2019) - valid for $0.075 M_{\odot}<M_{\star}<0.70 M_{\odot}$, and used to estimate $\log g$,

(v) Synthetic MARCS model spectra (for spectral fitting, interpolated to the resolution and wavelength grid of WiFeS)

(vi) MARCS model fluxes (for photometric fitting),

(vii) Stellar parallaxes from Gaia DR2,

(viii) The interstellar dust map from Leike \& Enßlin (2019),

(ix) A set of reference stellar standards with known parameters for testing and validation purposes (see Section B for details).

We found that least squares fitting between real and synthetic spectra alone consistently underestimated expected $\log g$ values of 


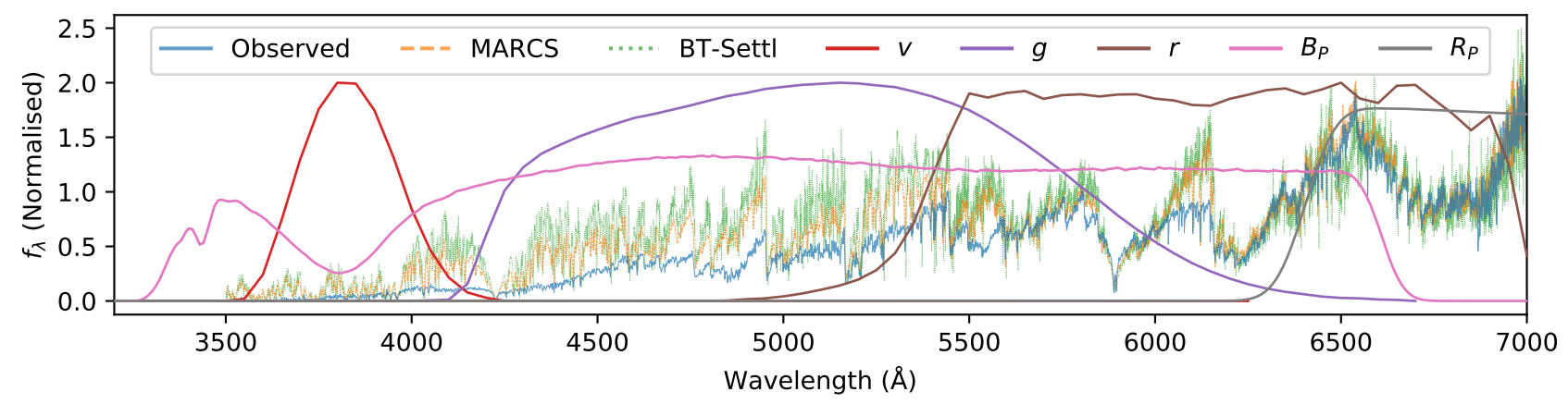

Figure 4. Observed WiFeS B3000 and R7000 spectra for GJ 447, along with a MARCS synthetic spectrum interpolated to the parameters from Mann et al. $2015\left(T_{\text {eff }}=3192 \mathrm{~K}, \log g=5.04,[\mathrm{Fe} / \mathrm{H}]=-0.02\right)$, and a PHOENIX/BT-Settl spectrum at the closest grid point available $\left(T_{\text {eff }}=3200 \mathrm{~K}, \log g=5.0,[\mathrm{Fe} / \mathrm{H}]\right.$ $=0.0$ ). SkyMapper $v, g, r$, and Gaia $B_{P}$ and $R_{P}$ filters are overplotted for reference. Note the severe model disagreement below $5400 \AA$.

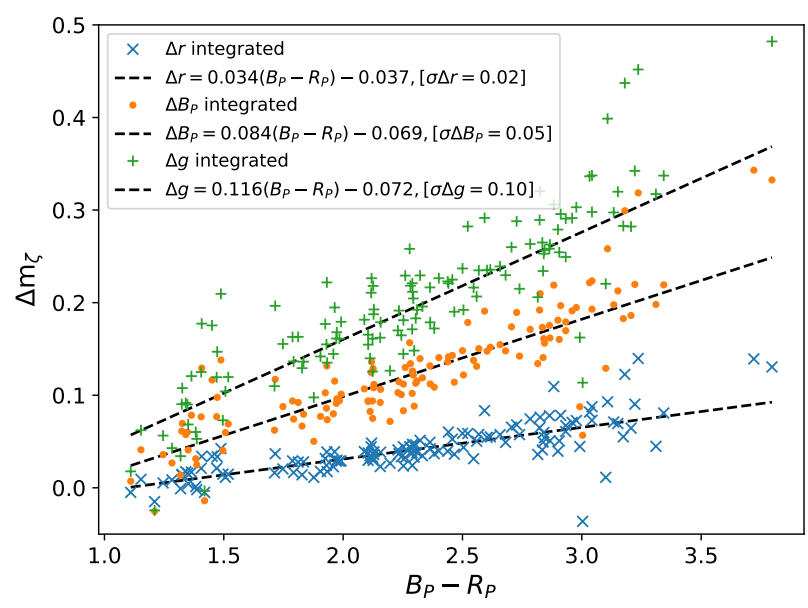

Figure 5. Gaia $B_{P}$, and SkyMapper $g r$ systematic offsets between integrated flux calibrated WiFeS spectra and MARCS model integrated spectra at literature parameters for our standard stars, plotted as a function of observed Gaia $B_{P}-R_{P}$. Stars redder in $B_{P}-R_{P}$ have systematically more flux at bluer wavelengths, with the best fit linear magnitude offset plotted for each filter, and the standard deviation in magnitude noted.

our sample by up to 0.3 dex - physical for a set of young stars, but not realistic for our overwhelmingly main sequence sample. To counter this, we calculate $\log g$ using the absolute $K_{S}$ band radius and mass relations of Mann et al. (2015) and Mann et al. (2019) ${ }^{8}$ respectively, and fix it during fitting. We then use a two step iterative procedure, with the first fit fixing $\log g$ to the value from empirical relations, and a second and final fit using our interim measured radius and a mass from Mann et al. (2019). All of our TESS targets fall within the stated $4<M_{K_{S}}<11$ limits for the mass relation. Although the relation is only valid for main sequence stars, we employ it with caution for two suspected young stars TOI 507 (TIC 348538431) and TOI 142 (425934411), both discussed in more detail in Section 6.5 , on the assumption that the resulting value of $\log g$ will still be more accurate than an unconstrained synthetic fit. Additionally, we

\footnotetext{
8 Calculated using the Python code available at: https://github.com/ awmann/M_-M_K-
}

suspect TOI 507 of being a near-equal mass binary, and as such treat it as 0.75 magnitudes fainter (or half as bright) for the purpose of using the relation, equivalent to determining the mass for only a single component.

While this now solves the $\log g$ issue, we are still left with two issues arising from the spectra themselves. The first is that certain wavelength regions of our MARCS model spectra are a poor match compared to our reference sample with known $T_{\text {eff }}, \log g$, and $[\mathrm{Fe} / \mathrm{H}]$ - particularly at cooler temperatures. As discussed in Section 4.1 , we account for this by using only spectra from the red arm of WiFeS with $\lambda>5400 \mathrm{~A}$, and masking out remaining regions with poor agreement.

The second remaining issue is that of the degeneracy between $T_{\text {eff }}$ and $[\mathrm{Fe} / \mathrm{H}]$ when fitting spectra. This effect is caused by both the temperature and metallicity influencing the strength of atmospheric molecular absorbers or opacity sources (predominantly $\mathrm{TiO}$ in the optical, but also various hydrides). What this means in practice is that there often isn't a single minimum or optimal set of atmospheric parameters when fitting synthetic spectra, but instead there exists a range of good fits (or even multiple minima) at different combinations of $T_{\text {eff }}$ and $[\mathrm{Fe} / \mathrm{H}]$ - possibly separated by several $100 \mathrm{~K}$ in $T_{\text {eff }}$ or several 0.1 dex in $[\mathrm{Fe} / \mathrm{H}]$.

In an attempt to overcome this, we include photometry from redder wavelengths that are less dominated by absorption than optical wavelengths, meaning that $T_{\text {eff }}$ and $[\mathrm{Fe} / \mathrm{H}]$ are less degenerate. While we do not have NIR spectra for our science or reference sample, we do have Gaia, SkyMapper, and 2MASS photometry in the form of $B_{P}, R_{P}, r, i, z, J, H$, and $K_{S}$ which together give us almost continuous wavelength coverage out to nearly $2.4 \mu \mathrm{m}$ and covers the bulk of stellar emission for our cool stars.

We thus modified our fitting methodology to also compute the uncertainty weighted residuals between observed and synthetic stellar photometry. In order to compare synthetic photometry to its observed equivalent we formulate the fit as follows:

$m_{\zeta, m}=\mathrm{BC}_{\zeta}\left(T_{\mathrm{eff}}, \log g,[\mathrm{Fe} / \mathrm{H}]\right)+m_{\text {bol }}$

where $m_{\zeta, m}$ is the model magnitude in filter $\zeta$; $\mathrm{BC}_{\zeta}$ is the bolometric correction (i.e. the total flux outside of a filter $\zeta$ ) as a function of $T_{\text {eff }}, \log g$, and $[\mathrm{Fe} / \mathrm{H}]$ in filter $\zeta$; and $m_{\text {bol }}$ is the apparent bolometric magnitude (i.e. the apparent magnitude of the star over all wavelengths). In this implementation $m_{\text {bol }}$ serves as a physically meaningful free parameter used to scale synthetic magni- 
tudes to their observed equivalents and ultimately allow computation of the apparent bolometric flux $f_{\text {bol }}$. This is done using the well tested bolometric-corrections ${ }^{9}$ software (Casagrande \& VandenBerg 2014, 2018a,b) to interpolate a grid of bolometric corrections from MARCS fluxes in different filters for the stellar parameters at each fitting call. By fitting for $m_{\text {bol }}$ and using bolometric corrections, we are thus directly able to compare an observed magnitude, $m_{\zeta, o}$, from Gaia, SkyMapper, or 2MASS directly with its MARCS synthetic equivalent. With $\log g$ fixed, we now have a three term fit in terms of $T_{\text {eff }},[\mathrm{Fe} / \mathrm{H}]$, and $m_{\text {bol }}$, the latter of which allows for direct computation of the bolometric flux (and thus the stellar radius).

This fitting procedure is equivalent to minimising the following relation (performed using the least_squares function from scipy's optimize module):

$R(\theta)=\sum_{i=1}^{M}\left(\frac{1}{C \sqrt{\chi_{f}^{2}}} \frac{f_{o, i}-f_{m, i}}{\sigma_{f_{o, i}}}\right)^{2}+\sum_{\zeta=1}^{N}\left(\frac{1}{\sqrt{\chi_{m}^{2}}} \frac{m_{\zeta, o}-\left(m_{\zeta, m}+\Delta m_{\zeta}\right)}{\sigma_{m_{\zeta}}}\right)$

with model uncertainties taken into account via:

$\sigma_{m_{\zeta}}=\sqrt{\sigma_{m_{\zeta, o}}^{2}+\sigma_{m_{\zeta, m}}^{2}}$

where $R(\theta)$ are the combined spectral and photometric squared residuals as a function of $\theta$, a vector of $\left.T_{\mathrm{eff}}, \log g,[\mathrm{Fe} / \mathrm{H}], m_{\mathrm{bol}}\right) ; M$ is the total number of spectral pixels, $i$ is the spectral pixel index, $f_{\mathrm{o}, \mathrm{i}}$ and $f_{\mathrm{m}, \mathrm{i}}$ are the observed and model spectral fluxes respectively at pixel $i$, normalised by their respective medians in the range $6200 \leqslant \lambda \leqslant 7000 \AA ; \sigma_{f_{\mathrm{o}, \mathrm{i}}}$ is the observed flux uncertainty at pixel $i ; N$ is the total number of photometric filters; $\zeta$ is the filter index, $m_{\zeta, o}$ and $m_{\zeta, m}$ are the observed and model magnitudes respectively in filter $\zeta ; \Delta m_{\zeta}$ is the systematic model magnitude offset in filter $\zeta$ (per Equation 3 for $r$ and $B_{P}$, and 0 for all other filters); $\sigma_{m_{\zeta, o}}$ and $\sigma_{m_{\zeta, m}}$ are the uncertainties on the observed and model magnitudes respectively, added in quadrature to give the total magnitude uncertainty $\sigma_{m_{\zeta}} ; \chi_{f}^{2}$ and $\chi_{m}^{2}$ are the global minimum $\chi^{2}$ values computed from the spectral and photometry residuals respectively (i.e. global fit using only R7000 spectra, without photometry, and a separate global photometric fit without spectra) used to normalise the two sets of residuals in the case of poor fits and place them on a similar scale; and $C$, set to 20 , is a constant used to account for the spectra having many more pixels than the number of photometric points. This value of $C$ was chosen by visually inspecting the residuals of our spectral fits and means that we assume, on average, every 20 spectral pixels are correlated and do not contain unique information.

We test the accuracy of our fitted $[\mathrm{Fe} / \mathrm{H}]$ using a set of cool star stellar standards in Figure 6. It is immediately clear that, despite the tight constraint on $T_{\text {eff }}$ that our broad wavelength coverage from photometry allows, we are unable to recover $[\mathrm{Fe} / \mathrm{H}]$ for our standard sample to better precision than our photometric $[\mathrm{Fe} / \mathrm{H}]$ relation from Section 3. Our fits systematically overpredict $[\mathrm{Fe} / \mathrm{H}]$ for the coolest stars in our sample, which might be similar to what was observed in Figure 3 of Rojas-Ayala et al. 2012 (using BT-Settl models), where they find even metal-rich models fail to reproduce the depth of certain features. This has also previously been observed for cool, metal-poor clusters when using evolutionary models (e.g. Joyce \& Chaboyer 2015), and observed for isochrones (e.g. Joyce \&

9 https://github.com/casaluca/bolometric-corrections
Chaboyer 2018). From this we conclude that a simple least squares fit to our medium resolution optical spectra, unweighted to $[\mathrm{Fe} / \mathrm{H}]$ sensitive regions, and using models with both known and unknown systematics is not sufficient to accurately determine $[\mathrm{Fe} / \mathrm{H}]$ for cool dwarfs.

Given this, it is clear a three parameter fit to $T_{\mathrm{eff}},[\mathrm{Fe} / \mathrm{H}]$, and $m_{\text {bol }}$ is unreasonable. Our final reported parameters are thus a two parameter fit to $T_{\mathrm{eff}}$, and $m_{\mathrm{bol}}$, fixing $[\mathrm{Fe} / \mathrm{H}]$ to the value from our relation in Section 3 for those stars falling within the $\left(B_{P}-R_{P}\right)$ range, and the mean value for the Solar Neighbourhood of $[\mathrm{Fe} / \mathrm{H}]$ $=-0.14$ (Schlaufman \& Laughlin 2010) for stars outside this range, or suspected of binarity or being young. To further account for both model and zeropoint uncertainties, we add a $1 \%$ flux uncertainty in quadrature with our fitted statistical uncertainties on $m_{\text {bol }}$. Our standard star $T_{\text {eff }}$ recovery for the two parameter fit is shown in Figure 7.

$2 \quad$ We compute the apparent bolometric flux $f_{\text {bol }}$ from our fitted value of $m_{\text {bol }}$ using Equation 3 from Casagrande \& VandenBerg (2018a), from which we then compute the stellar radius $R_{\star}$. Figure 8 shows a comparison between our radii and those from our interferometric standard sample, and final values for TESS science targets and stellar standards are reported in Tables 2 and B2 respectively.

\section{CANDIDATE PLANET PARAMETERS}

\subsection{Transit Light Curve Analysis}

We now present results for all TOIs not ruled out as false positives (e.g. due to background stars, or eclipsing binaries) by the TESS Team and exoplanet community, as listed on the NASA ExoFOPTESS website.

Transit light curves for targets across all TESS sectors were downloaded from NASA's Mikulski Archive for Space Telescopes (MAST) service. For all high-cadence data, we used the Pre-search Data Conditioning Simple Aperture Photometry (PDCSAP) fluxes, which have already had some measure of processing to remove systematics.

All light curves were downloaded and manipulated using the python package LightKurve (Lightkurve Collaboration et al. 2018).

Many stars in our sample show some amount of stellar variability, with periods ranging from days to many weeks. We remove this using LightKurve's flatten function, which applies a SavitzkyGolay filter (Savitzky \& Golay 1964) to the data to remove low frequency trends. When applying the filter we mask out all planetary transits by known TOIs. Once flattened, the light curves are then phase folded using either the period provided by NASA ExoFOPTESS (for most stars), or our own fitted period (for stars revisited in the TESS extended mission whose long time baseline reveals the ExoFOP-TESS period to be incorrect). We use the provided measurement of transit duration to select only photometry from the transit itself, plus $10 \%$ of a duration either side for use in model fitting.

Model fitting is implemented using the python package BATMAN (Kreidberg 2015), which is capable of generating model transit light curves for a given set of orbital elements (scaled by the stellar radius $R_{\star}$ ) and limb darkening coefficients. We use a four term limb darkening law, interpolating the PHOENIX grid provided by Claret (2017) using values of $T_{\text {eff }}$ and $\log g$ from Table 2. The resulting coefficients are in Table D1.

Transit photometry alone is not sufficient to uniquely constrain 
Table 2. Final results for TESS candidate exoplanet hosts

\begin{tabular}{|c|c|c|c|c|c|c|c|c|c|c|}
\hline TOI & TIC & $\begin{array}{l}T_{\text {eff }} \\
(\mathrm{K})\end{array}$ & $\log g$ & {$[\mathrm{Fe} / \mathrm{H}]$} & $\begin{array}{c}M \\
\left(M_{\odot}\right)\end{array}$ & $\begin{array}{c}R_{\star} \\
\left(R_{\odot}\right)\end{array}$ & $m_{\mathrm{bol}}$ & $\begin{array}{c}f_{\text {bol }} \\
\left(10^{-12} \operatorname{ergs~s}^{-1} \mathrm{~cm}^{-2}\right)\end{array}$ & $\begin{array}{c}\mathrm{EW}(\mathrm{H} \alpha) \\
\AA\end{array}$ & $\log R_{\mathrm{HK}}^{\prime}$ \\
\hline 136 & 410153553 & $2988 \pm 30$ & $5.06 \pm 0.02$ & - & $0.155 \pm 0.004$ & $0.192 \pm 0.004$ & $12.05 \pm 0.01$ & $384.4 \pm 3.9$ & -0.05 & -5.37 \\
\hline 540 & 200322593 & $3104 \pm 30$ & $5.07 \pm 0.02$ & -0.10 & $0.164 \pm 0.004$ & $0.197 \pm 0.004$ & $11.71 \pm 0.01$ & $528.3 \pm 5.3$ & 2.49 & - \\
\hline 256 & 92226327 & $3150 \pm 30$ & $5.01 \pm 0.02$ & -0.13 & $0.182 \pm 0.005$ & $0.220 \pm 0.004$ & $11.55 \pm 0.01$ & $611.8 \pm 6.1$ & -0.22 & -5.53 \\
\hline 203 & 259962054 & $3169 \pm 30$ & $5.01 \pm 0.02$ & -0.07 & $0.200 \pm 0.005$ & $0.232 \pm 0.004$ & $12.49 \pm 0.01$ & $255.4 \pm 2.6$ & 0.56 & -4.98 \\
\hline 507 & 348538431 & $3279 \pm 30$ & $4.76 \pm 0.02$ & - & $0.383 \pm 0.010$ & $0.424 \pm 0.008$ & $14.28 \pm 0.01$ & $49.2 \pm 0.5$ & 2.24 & -4.49 \\
\hline 910 & 369327947 & $3282 \pm 30$ & $4.97 \pm 0.02$ & -0.04 & $0.262 \pm 0.008$ & $0.278 \pm 0.005$ & $10.46 \pm 0.01$ & $1656.2 \pm 16.6$ & -0.27 & -5.47 \\
\hline 210 & 141608198 & $3284 \pm 30$ & $4.90 \pm 0.02$ & 0.21 & $0.312 \pm 0.008$ & $0.326 \pm 0.006$ & $12.78 \pm 0.01$ & $195.8 \pm 2.0$ & -0.23 & -5.84 \\
\hline 122 & 231702397 & $3326 \pm 30$ & $4.86 \pm 0.02$ & -0.07 & $0.316 \pm 0.008$ & $0.345 \pm 0.006$ & $13.42 \pm 0.01$ & $109.3 \pm 1.1$ & -0.21 & - \\
\hline 455 & 98796344 & $3330 \pm 30$ & $4.97 \pm 0.02$ & -0.27 & $0.248 \pm 0.006$ & $0.271 \pm 0.005$ & $9.16 \pm 0.01$ & $5507.5 \pm 54.8$ & -0.29 & -5.39 \\
\hline 732 & 36724087 & $3354 \pm 30$ & $4.83 \pm 0.02$ & 0.13 & $0.364 \pm 0.009$ & $0.382 \pm 0.007$ & $10.91 \pm 0.01$ & $1103.5 \pm 11.0$ & -0.23 & -5.55 \\
\hline 674 & 158588995 & $3355 \pm 30$ & $4.77 \pm 0.02$ & - & $0.419 \pm 0.010$ & $0.443 \pm 0.008$ & $12.19 \pm 0.01$ & $338.7 \pm 3.4$ & -0.45 & -5.58 \\
\hline 406 & 153065527 & $3369 \pm 30$ & $4.83 \pm 0.02$ & 0.21 & $0.380 \pm 0.009$ & $0.392 \pm 0.007$ & $11.58 \pm 0.01$ & $594.6 \pm 5.9$ & -0.15 & -5.40 \\
\hline 175 & 307210830 & $3381 \pm 30$ & $4.94 \pm 0.02$ & -0.24 & $0.293 \pm 0.007$ & $0.304 \pm 0.005$ & $9.78 \pm 0.01$ & $3099.8 \pm 31.1$ & -0.31 & -5.47 \\
\hline 782 & 429358906 & $3390 \pm 30$ & $4.81 \pm 0.02$ & 0.25 & $0.401 \pm 0.010$ & $0.413 \pm 0.008$ & $12.57 \pm 0.01$ & $237.3 \pm 2.4$ & -0.37 & -6.38 \\
\hline 244 & 118327550 & $3422 \pm 30$ & $4.82 \pm 0.02$ & 0.10 & $0.402 \pm 0.010$ & $0.407 \pm 0.007$ & $10.69 \pm 0.01$ & $1349.2 \pm 13.5$ & -0.30 & -5.36 \\
\hline 789 & 300710077 & $3434 \pm 30$ & $4.86 \pm 0.02$ & -0.16 & $0.360 \pm 0.009$ & $0.371 \pm 0.007$ & $12.34 \pm 0.01$ & $294.0 \pm 2.9$ & -0.28 & - \\
\hline 654 & 35009898 & $3436 \pm 30$ & $4.77 \pm 0.02$ & 0.07 & $0.425 \pm 0.010$ & $0.445 \pm 0.008$ & $12.56 \pm 0.01$ & $239.6 \pm 2.4$ & -0.33 & -8.10 \\
\hline 864 & 231728511 & $3452 \pm 30$ & $4.82 \pm 0.02$ & -0.10 & $0.392 \pm 0.010$ & $0.403 \pm 0.007$ & $11.86 \pm 0.01$ & $460.0 \pm 4.6$ & -0.37 & -5.21 \\
\hline 562 & 413248763 & $3456 \pm 30$ & $4.88 \pm 0.02$ & -0.22 & $0.347 \pm 0.008$ & $0.353 \pm 0.006$ & $9.11 \pm 0.01$ & $5758.7 \pm 57.3$ & -0.25 & -5.93 \\
\hline 486 & 260708537 & $3467 \pm 30$ & $4.81 \pm 0.02$ & 0.03 & $0.424 \pm 0.010$ & $0.424 \pm 0.007$ & $9.74 \pm 0.01$ & $3239.2 \pm 32.4$ & -0.40 & -5.39 \\
\hline 700 & 150428135 & $3467 \pm 30$ & $4.80 \pm 0.02$ & - & $0.416 \pm 0.010$ & $0.426 \pm 0.007$ & $11.28 \pm 0.01$ & $783.3 \pm 7.8$ & -0.35 & -5.36 \\
\hline 521 & 27649847 & $3468 \pm 30$ & $4.80 \pm 0.02$ & -0.01 & $0.416 \pm 0.010$ & $0.424 \pm 0.008$ & $12.74 \pm 0.01$ & $203.5 \pm 2.0$ & -0.30 & -5.49 \\
\hline 1078 & 370133522 & $3486 \pm 30$ & $4.83 \pm 0.02$ & -0.24 & $0.383 \pm 0.009$ & $0.392 \pm 0.007$ & $10.51 \pm 0.01$ & $1583.2 \pm 15.8$ & -0.34 & -6.54 \\
\hline 912 & 406941612 & $3488 \pm 30$ & $4.80 \pm 0.02$ & -0.03 & $0.424 \pm 0.010$ & $0.427 \pm 0.007$ & $10.87 \pm 0.01$ & $1143.7 \pm 11.4$ & -0.31 & -5.19 \\
\hline 270 & 259377017 & $3493 \pm 30$ & $4.88 \pm 0.02$ & -0.25 & $0.364 \pm 0.009$ & $0.361 \pm 0.006$ & $10.90 \pm 0.01$ & $1111.3 \pm 11.1$ & -0.29 & -5.40 \\
\hline 696 & 77156829 & $3496 \pm 30$ & $4.92 \pm 0.02$ & -0.45 & $0.321 \pm 0.008$ & $0.327 \pm 0.006$ & $10.84 \pm 0.01$ & $1170.5 \pm 11.7$ & -0.22 & -6.08 \\
\hline 269 & 220479565 & $3518 \pm 30$ & $4.83 \pm 0.02$ & -0.25 & $0.391 \pm 0.010$ & $0.396 \pm 0.007$ & $12.69 \pm 0.01$ & $214.2 \pm 2.1$ & -0.30 & - \\
\hline 233 & 415969908 & $3527 \pm 30$ & $4.88 \pm 0.02$ & -0.31 & $0.371 \pm 0.009$ & $0.365 \pm 0.006$ & $11.72 \pm 0.01$ & $522.8 \pm 5.2$ & -0.31 & -5.21 \\
\hline 698 & 141527579 & $3540 \pm 30$ & $4.76 \pm 0.02$ & 0.04 & $0.473 \pm 0.011$ & $0.474 \pm 0.008$ & $12.49 \pm 0.01$ & $255.5 \pm 2.5$ & -0.39 & -5.12 \\
\hline 731 & 34068865 & $3543 \pm 30$ & $4.77 \pm 0.02$ & -0.03 & $0.458 \pm 0.011$ & $0.463 \pm 0.008$ & $8.41 \pm 0.01$ & $11008.1 \pm 109.5$ & -0.39 & -5.24 \\
\hline 1201 & 29960110 & $3546 \pm 30$ & $4.75 \pm 0.02$ & 0.11 & $0.484 \pm 0.012$ & $0.488 \pm 0.008$ & $11.31 \pm 0.01$ & $757.8 \pm 7.6$ & -0.13 & -4.74 \\
\hline 756 & 73649615 & $3581 \pm 30$ & $4.71 \pm 0.02$ & 0.11 & $0.509 \pm 0.012$ & $0.522 \pm 0.009$ & $12.90 \pm 0.01$ & $175.4 \pm 1.7$ & -0.42 & -4.90 \\
\hline 704 & 260004324 & $3596 \pm 30$ & $4.69 \pm 0.02$ & -0.05 & $0.506 \pm 0.012$ & $0.533 \pm 0.009$ & $10.54 \pm 0.01$ & $1540.8 \pm 15.4$ & -0.41 & -5.01 \\
\hline 177 & 262530407 & $3613 \pm 30$ & $4.70 \pm 0.02$ & - & $0.519 \pm 0.013$ & $0.532 \pm 0.009$ & $9.91 \pm 0.01$ & $2762.3 \pm 27.5$ & 0.00 & -4.45 \\
\hline 797 & 271596225 & $3618 \pm 30$ & $4.76 \pm 0.02$ & - & $0.475 \pm 0.011$ & $0.476 \pm 0.008$ & $12.13 \pm 0.01$ & $356.5 \pm 3.5$ & -0.45 & -4.92 \\
\hline 727 & 149788158 & $3634 \pm 30$ & $4.74 \pm 0.02$ & -0.11 & $0.499 \pm 0.012$ & $0.501 \pm 0.008$ & $11.42 \pm 0.01$ & $686.3 \pm 6.8$ & -0.40 & -5.02 \\
\hline 142 & 425934411 & $3647 \pm 30$ & $4.55 \pm 0.02$ & - & $0.594 \pm 0.016$ & $0.675 \pm 0.016$ & $15.09 \pm 0.01$ & $23.4 \pm 0.2$ & 0.98 & -4.25 \\
\hline 663 & 54962195 & $3658 \pm 30$ & $4.72 \pm 0.02$ & -0.10 & $0.514 \pm 0.012$ & $0.521 \pm 0.009$ & $12.18 \pm 0.01$ & $341.4 \pm 3.4$ & -0.49 & - \\
\hline 234 & 12423815 & $3668 \pm 30$ & $4.70 \pm 0.02$ & 0.13 & $0.545 \pm 0.015$ & $0.546 \pm 0.013$ & $14.99 \pm 0.01$ & $25.7 \pm 0.3$ & -0.51 & -4.97 \\
\hline 620 & 296739893 & $3669 \pm 30$ & $4.70 \pm 0.02$ & 0.19 & $0.547 \pm 0.014$ & $0.547 \pm 0.009$ & $10.62 \pm 0.01$ & $1437.2 \pm 14.3$ & -0.45 & -5.07 \\
\hline 672 & 151825527 & $3678 \pm 30$ & $4.67 \pm 0.02$ & 0.01 & $0.544 \pm 0.013$ & $0.563 \pm 0.009$ & $12.08 \pm 0.01$ & $375.7 \pm 3.7$ & -0.34 & -4.62 \\
\hline 873 & 237920046 & $3682 \pm 30$ & $4.72 \pm 0.02$ & -0.09 & $0.521 \pm 0.013$ & $0.519 \pm 0.009$ & $12.55 \pm 0.01$ & $243.2 \pm 2.4$ & -0.39 & -4.81 \\
\hline 714 & 219195044 & $3698 \pm 30$ & $4.77 \pm 0.02$ & -0.33 & $0.474 \pm 0.011$ & $0.468 \pm 0.008$ & $11.98 \pm 0.01$ & $410.0 \pm 4.1$ & -0.47 & -5.22 \\
\hline 134 & 234994474 & $3722 \pm 30$ & $4.60 \pm 0.02$ & - & $0.590 \pm 0.015$ & $0.633 \pm 0.010$ & $9.65 \pm 0.01$ & $3512.5 \pm 35.0$ & -0.47 & -4.69 \\
\hline 468 & 33521996 & $3738 \pm 30$ & $4.60 \pm 0.02$ & - & $0.588 \pm 0.015$ & $0.634 \pm 0.012$ & $13.75 \pm 0.01$ & $80.4 \pm 0.8$ & -0.55 & -4.93 \\
\hline 785 & 374829238 & $3740 \pm 30$ & $4.67 \pm 0.02$ & -0.02 & $0.559 \pm 0.014$ & $0.572 \pm 0.009$ & $11.92 \pm 0.01$ & $432.7 \pm 4.3$ & -0.39 & -4.52 \\
\hline 552 & 44737596 & $3742 \pm 30$ & $4.65 \pm 0.02$ & 0.21 & $0.579 \pm 0.014$ & $0.594 \pm 0.011$ & $14.19 \pm 0.01$ & $53.6 \pm 0.5$ & -0.42 & -4.19 \\
\hline 904 & 261257684 & $3752 \pm 30$ & $4.71 \pm 0.02$ & -0.17 & $0.533 \pm 0.013$ & $0.537 \pm 0.009$ & $11.28 \pm 0.01$ & $778.7 \pm 7.8$ & -0.39 & -4.60 \\
\hline 741 & 359271092 & $3763 \pm 30$ & $4.73 \pm 0.02$ & -0.17 & $0.527 \pm 0.013$ & $0.520 \pm 0.008$ & $8.12 \pm 0.01$ & $14329.1 \pm 142.6$ & -0.46 & -5.03 \\
\hline 198 & 12421862 & $3770 \pm 30$ & $4.86 \pm 0.02$ & - & $0.447 \pm 0.011$ & $0.413 \pm 0.007$ & $10.39 \pm 0.01$ & $1769.6 \pm 17.7$ & -0.42 & -5.14 \\
\hline 442 & 70899085 & $3831 \pm 30$ & $4.63 \pm 0.02$ & 0.09 & $0.598 \pm 0.015$ & $0.620 \pm 0.010$ & $11.17 \pm 0.01$ & $867.7 \pm 8.6$ & -0.40 & -4.53 \\
\hline 557 & 55488511 & $3845 \pm 30$ & $4.67 \pm 0.02$ & -0.15 & $0.570 \pm 0.014$ & $0.581 \pm 0.009$ & $12.09 \pm 0.01$ & $371.3 \pm 3.7$ & -0.51 & -4.69 \\
\hline 870 & 219229644 & $3847 \pm 30$ & $4.63 \pm 0.02$ & 0.11 & $0.601 \pm 0.015$ & $0.618 \pm 0.010$ & $11.21 \pm 0.01$ & $836.3 \pm 8.3$ & -0.38 & -4.54 \\
\hline 551 & 192826603 & $3871 \pm 30$ & $4.67 \pm 0.02$ & -0.28 & $0.563 \pm 0.014$ & $0.577 \pm 0.010$ & $14.35 \pm 0.01$ & $46.4 \pm 0.5$ & -0.54 & -4.60 \\
\hline 876 & 32497972 & $3888 \pm 30$ & $4.63 \pm 0.02$ & -0.08 & $0.595 \pm 0.015$ & $0.615 \pm 0.010$ & $11.98 \pm 0.01$ & $410.9 \pm 4.1$ & -0.55 & -4.85 \\
\hline 532 & 144700903 & $3903 \pm 30$ & $4.62 \pm 0.02$ & 0.07 & $0.610 \pm 0.015$ & $0.630 \pm 0.011$ & $13.09 \pm 0.01$ & $147.8 \pm 1.5$ & -0.68 & -5.39 \\
\hline 1075 & 351601843 & $3916 \pm 30$ & $4.69 \pm 0.02$ & - & $0.575 \pm 0.014$ & $0.571 \pm 0.009$ & $11.59 \pm 0.01$ & $588.2 \pm 5.9$ & -0.50 & -4.66 \\
\hline 833 & 362249359 & $3945 \pm 30$ & $4.65 \pm 0.02$ & -0.13 & $0.595 \pm 0.015$ & $0.601 \pm 0.009$ & $10.60 \pm 0.01$ & $1458.0 \pm 14.5$ & -0.49 & -4.49 \\
\hline 702 & 237914496 & $3966 \pm 30$ & $4.69 \pm 0.02$ & -0.24 & $0.577 \pm 0.014$ & $0.566 \pm 0.009$ & $12.24 \pm 0.01$ & $323.3 \pm 3.2$ & -0.56 & -4.65 \\
\hline 555 & 170849515 & $3993 \pm 30$ & $4.63 \pm 0.02$ & -0.06 & $0.615 \pm 0.017$ & $0.626 \pm 0.015$ & $15.25 \pm 0.01$ & $20.1 \pm 0.2$ & -0.40 & -4.76 \\
\hline 285 & 220459976 & $3995 \pm 30$ & $4.62 \pm 0.02$ & 0.00 & $0.625 \pm 0.015$ & $0.643 \pm 0.010$ & $12.61 \pm 0.01$ & $229.2 \pm 2.3$ & -0.60 & -4.71 \\
\hline 475 & 100608026 & $4003 \pm 30$ & $4.66 \pm 0.02$ & -0.18 & $0.601 \pm 0.015$ & $0.599 \pm 0.009$ & $11.29 \pm 0.01$ & $775.1 \pm 7.7$ & -0.54 & -4.62 \\
\hline 253 & 322063810 & $4039 \pm 30$ & $4.65 \pm 0.02$ & - & $0.617 \pm 0.015$ & $0.618 \pm 0.009$ & $9.79 \pm 0.01$ & $3087.3 \pm 30.8$ & -0.60 & -4.87 \\
\hline 435 & 44647437 & $4079 \pm 30$ & $4.64 \pm 0.02$ & -0.13 & $0.621 \pm 0.015$ & $0.627 \pm 0.010$ & $13.34 \pm 0.01$ & $116.9 \pm 1.2$ & -0.56 & -4.57 \\
\hline
\end{tabular}



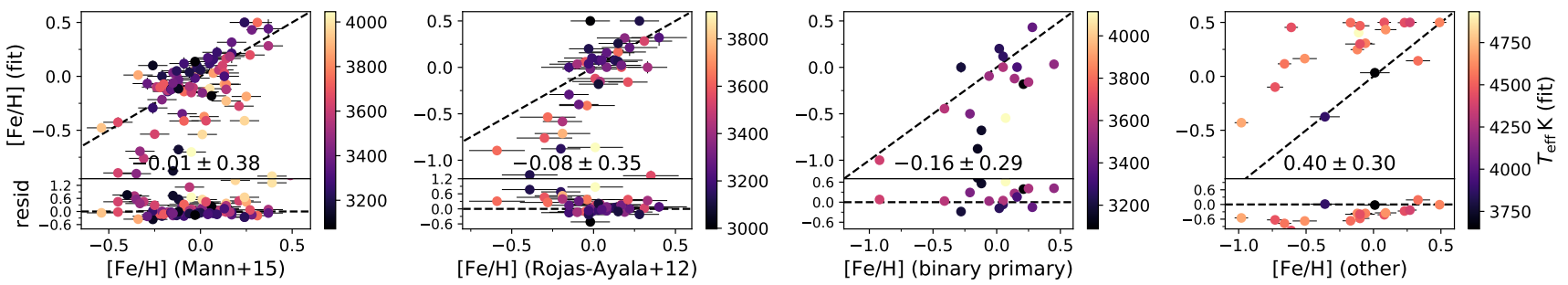

Figure 6. $[\mathrm{Fe} / \mathrm{H}]$ recovery for our 3 parameter fit in $T_{\mathrm{eff}},[\mathrm{Fe} / \mathrm{H}], m_{\text {bol }}$ for our four sets of $[\mathrm{Fe} / \mathrm{H}]$ standards: Mann et al. 2015, Rojas-Ayala et al. 2012, primary star $[\mathrm{Fe} / \mathrm{H}]$ for cool dwarfs in binaries, and mid-K dwarfs. The median and standard deviation of each set of residuals is annotated. Note the inability of the 3 parameter fit to reliably recover $[\mathrm{Fe} / \mathrm{H}]$, with the scatter on our recovered $[\mathrm{Fe} / \mathrm{H}]$ for the binary sample (the most reliable set of $[\mathrm{Fe} / \mathrm{H}]$ standards) being larger than the scatter on our photometric $[\mathrm{Fe} / \mathrm{H}]$ relation.
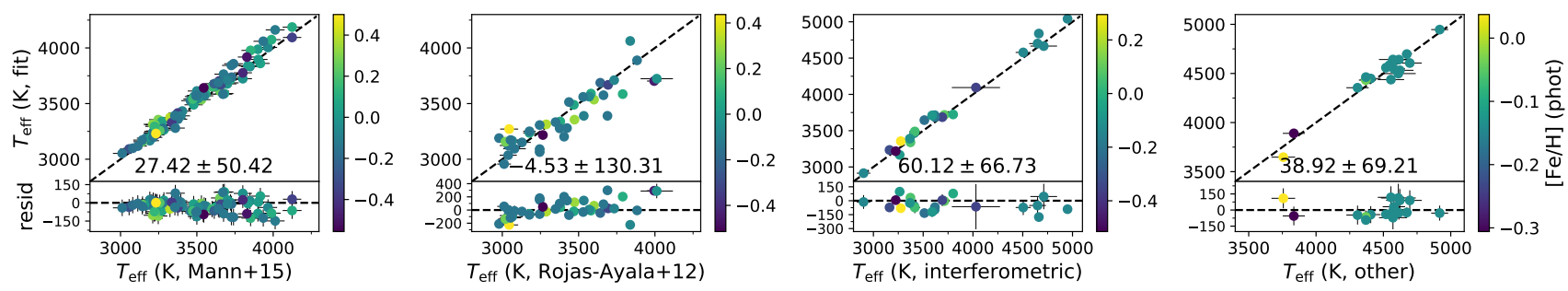

Figure 7. $T_{\text {eff }}$ recovery for our 2 parameter fit in $T_{\text {eff }}$, and $m_{\text {bol }}$ for our four sets of $T_{\text {eff }}$ standards: Mann et al. 2015, Rojas-Ayala et al. 2012, interferometry, and mid-K dwarfs. $[\mathrm{Fe} / \mathrm{H}]$ is from our photometric $[\mathrm{Fe} / \mathrm{H}]$ relation where appropriate, or fixed to the mean Solar Neighbourhood $[\mathrm{Fe} / \mathrm{H}]$ if not. The median and standard deviation of each set of residuals is annotated (note that these values have not yet been corrected for the systematic, as discussed in Section 6.2).

Table 2 - continued Final results for TESS candidate exoplanet hosts

\begin{tabular}{|c|c|c|c|c|c|c|c|c|c|c|}
\hline TOI & TIC & $\begin{array}{l}T_{\text {eff }} \\
(\mathrm{K})\end{array}$ & $\log g$ & {$[\mathrm{Fe} / \mathrm{H}]$} & $\begin{array}{c}M \\
\left(M_{\odot}\right)\end{array}$ & $\begin{array}{c}R_{\star} \\
\left(R_{\odot}\right)\end{array}$ & $m_{\text {bol }}$ & $\begin{array}{c}f_{\text {bol }} \\
\left(10^{-12} \mathrm{ergs} \mathrm{s}^{-1} \mathrm{~cm}^{-2}\right)\end{array}$ & $\begin{array}{c}\mathrm{EW}(\mathrm{H} \alpha) \\
\AA\end{array}$ & $\log R_{\mathrm{HK}}^{\prime}$ \\
\hline 1082 & 261108236 & $4096 \pm 30$ & $4.65 \pm 0.02$ & -0.24 & $0.610 \pm 0.015$ & $0.609 \pm 0.009$ & $12.31 \pm 0.01$ & $302.8 \pm 3.0$ & -0.61 & -4.76 \\
\hline 260 & 37749396 & $4097 \pm 30$ & $4.70 \pm 0.02$ & -0.28 & $0.598 \pm 0.015$ & $0.575 \pm 0.009$ & $8.96 \pm 0.01$ & $6614.9 \pm 65.9$ & -0.60 & -4.75 \\
\hline 761 & 165317334 & $4121 \pm 30$ & $4.64 \pm 0.02$ & -0.15 & $0.623 \pm 0.015$ & $0.629 \pm 0.010$ & $11.34 \pm 0.01$ & $740.9 \pm 7.4$ & -0.60 & -4.49 \\
\hline 249 & 179985715 & $4128 \pm 30$ & $4.71 \pm 0.02$ & - & $0.589 \pm 0.015$ & $0.561 \pm 0.008$ & $11.70 \pm 0.01$ & $531.3 \pm 5.3$ & -0.56 & -4.61 \\
\hline 806 & 33831980 & $4137 \pm 30$ & $4.68 \pm 0.02$ & -0.29 & $0.607 \pm 0.015$ & $0.592 \pm 0.009$ & $12.42 \pm 0.01$ & $274.5 \pm 2.7$ & -0.62 & -4.65 \\
\hline 1216 & 141527965 & $4217 \pm 30$ & $4.61 \pm 0.02$ & -0.13 & $0.649 \pm 0.016$ & $0.664 \pm 0.010$ & $11.98 \pm 0.01$ & $410.5 \pm 4.1$ & -0.67 & -4.68 \\
\hline 302 & 229111835 & $4227 \pm 30$ & $4.59 \pm 0.02$ & -0.03 & $0.661 \pm 0.016$ & $0.684 \pm 0.011$ & $13.36 \pm 0.01$ & $115.2 \pm 1.2$ & -0.73 & -4.60 \\
\hline 656 & 36734222 & $4241 \pm 30$ & $4.58 \pm 0.02$ & -0.07 & $0.662 \pm 0.016$ & $0.690 \pm 0.010$ & $11.57 \pm 0.01$ & $595.9 \pm 6.0$ & -0.61 & -4.46 \\
\hline 1130 & 254113311 & $4275 \pm 30$ & $4.55 \pm 0.02$ & -0.12 & $0.670 \pm 0.017$ & $0.716 \pm 0.011$ & $10.60 \pm 0.01$ & $1463.9 \pm 14.6$ & -0.73 & -4.78 \\
\hline 133 & 219338557 & $4276 \pm 30$ & $4.64 \pm 0.02$ & -0.22 & $0.646 \pm 0.016$ & $0.639 \pm 0.009$ & $10.45 \pm 0.01$ & $1673.5 \pm 16.7$ & -0.71 & -5.06 \\
\hline 544 & 50618703 & $4292 \pm 30$ & $4.66 \pm 0.02$ & -0.24 & $0.641 \pm 0.016$ & $0.623 \pm 0.009$ & $10.13 \pm 0.01$ & $2253.9 \pm 22.4$ & -0.64 & -4.56 \\
\hline 836 & 440887364 & $4308 \pm 30$ & $4.62 \pm 0.02$ & -0.15 & $0.658 \pm 0.016$ & $0.659 \pm 0.009$ & $9.12 \pm 0.01$ & $5710.9 \pm 56.9$ & -0.68 & -4.61 \\
\hline 240 & 101948569 & $4333 \pm 30$ & $4.59 \pm 0.02$ & - & $0.676 \pm 0.017$ & $0.693 \pm 0.010$ & $11.16 \pm 0.01$ & $876.1 \pm 8.8$ & -0.68 & -4.60 \\
\hline 713 & 167600516 & $4340 \pm 30$ & $4.64 \pm 0.02$ & -0.25 & $0.648 \pm 0.016$ & $0.637 \pm 0.009$ & $11.17 \pm 0.01$ & $868.0 \pm 8.7$ & -0.74 & -5.09 \\
\hline 900 & 210873792 & $4347 \pm 30$ & $4.63 \pm 0.02$ & -0.23 & $0.653 \pm 0.016$ & $0.649 \pm 0.010$ & $12.32 \pm 0.01$ & $300.0 \pm 3.0$ & -0.71 & -5.46 \\
\hline 493 & 19025965 & $4360 \pm 30$ & $4.59 \pm 0.02$ & -0.05 & $0.677 \pm 0.017$ & $0.690 \pm 0.010$ & $11.92 \pm 0.01$ & $434.7 \pm 4.3$ & -0.73 & -4.87 \\
\hline 178 & 251848941 & $4366 \pm 30$ & $4.64 \pm 0.02$ & - & $0.649 \pm 0.016$ & $0.639 \pm 0.009$ & $10.91 \pm 0.01$ & $1095.3 \pm 10.9$ & -0.75 & -4.85 \\
\hline 875 & 14165625 & $4404 \pm 30$ & $4.59 \pm 0.02$ & -0.13 & $0.675 \pm 0.017$ & $0.689 \pm 0.010$ & $11.85 \pm 0.01$ & $463.0 \pm 4.6$ & -0.69 & -4.57 \\
\hline 711 & 38510224 & $4433 \pm 30$ & $4.64 \pm 0.02$ & - & $0.653 \pm 0.016$ & $0.638 \pm 0.009$ & $12.22 \pm 0.01$ & $327.6 \pm 3.3$ & -0.73 & -4.77 \\
\hline 139 & 62483237 & $4434 \pm 30$ & $4.61 \pm 0.02$ & - & $0.673 \pm 0.016$ & $0.674 \pm 0.009$ & $9.88 \pm 0.01$ & $2827.2 \pm 28.2$ & -0.69 & -4.47 \\
\hline 1073 & 158297421 & $4481 \pm 30$ & $4.62 \pm 0.02$ & - & $0.666 \pm 0.017$ & $0.664 \pm 0.014$ & $14.09 \pm 0.01$ & $58.6 \pm 0.6$ & -0.69 & -4.60 \\
\hline 929 & 175532955 & $4482 \pm 30$ & $4.59 \pm 0.02$ & - & $0.684 \pm 0.017$ & $0.693 \pm 0.010$ & $11.92 \pm 0.01$ & $432.7 \pm 4.3$ & -0.79 & -10.15 \\
\hline 969 & 280437559 & $4503 \pm 30$ & $4.59 \pm 0.02$ & - & $0.682 \pm 0.017$ & $0.693 \pm 0.010$ & $11.06 \pm 0.01$ & $960.4 \pm 9.6$ & -0.72 & -4.60 \\
\hline 932 & 260417932 & $4508 \pm 30$ & $4.58 \pm 0.02$ & - & $0.689 \pm 0.017$ & $0.705 \pm 0.010$ & $11.23 \pm 0.01$ & $817.5 \pm 8.1$ & -0.80 & -5.22 \\
\hline 1067 & 201642601 & $4510 \pm 30$ & $4.57 \pm 0.02$ & - & $0.698 \pm 0.018$ & $0.721 \pm 0.012$ & $13.61 \pm 0.01$ & $91.7 \pm 0.9$ & -0.76 & 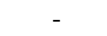 \\
\hline 279 & 122613513 & $4512 \pm 30$ & $4.61 \pm 0.02$ & - & $0.677 \pm 0.017$ & $0.673 \pm 0.009$ & $11.03 \pm 0.01$ & $986.9 \pm 9.8$ & -0.76 & -4.61 \\
\hline 129 & 201248411 & $4569 \pm 30$ & $4.57 \pm 0.02$ & - & $0.697 \pm 0.018$ & $0.721 \pm 0.010$ & $10.42 \pm 0.01$ & $1721.3 \pm 17.2$ & -0.77 & -4.50 \\
\hline 824 & 193641523 & $4589 \pm 30$ & $4.60 \pm 0.02$ & - & $0.688 \pm 0.018$ & $0.691 \pm 0.009$ & $10.57 \pm 0.01$ & $1505.7 \pm 15.0$ & -0.81 & -4.76 \\
\hline
\end{tabular}




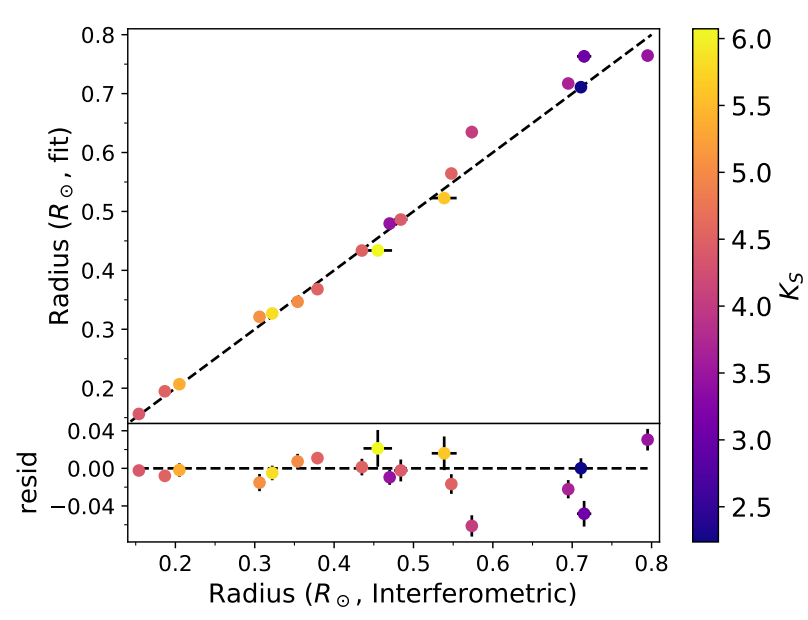

Figure 8. Radius comparison for those targets with interfometric radii to better than 5\% precision. The median distance precision for these targets is $0.04 \%$. We find generally good agreement between literature measurements and our own, though noting that the brightness of this sample (see apparent 2MASS $K_{S}$ magnitude on the colour bar) results in photometry that is either saturated or has lower precision and thus may be the cause of some of the scatter observed.

the planet orbit and radius when fitting for the scaled semi-major axis $a_{R_{\star}}=\frac{a}{R_{\star}}$, the planetary radius ratio $R_{P, R_{\star}}=\frac{R_{P}}{R_{\star}}$, the inclination $i$, the eccentricity $e$, and the longitude of periastron $\omega$ (Kipping 2008). While we can use our measurements of $M_{\star}, R_{\star}$, and $T$ to constrain the semi-major axis of a circular orbit (Equation 7), we do not have the precision required to fit for eccentric orbits. As such, we fix $e=0$ and $\omega=0$ during our fit, and include our calculated value $a_{R_{\star}, c}$ - the value $a_{R_{\star}}$ assuming a circular orbit, as a prior during fitting. In cases where $e \sim 0$, we expect the fitted semi-major axis $a_{R_{\star}, f}$ to approach $a_{R_{\star}, c}$. For cases with a discrepancy between the two, we flag the planet as an indication of a possibly eccentric orbit in Table 3.

This measured semi-major axis, calculated using our Mann et al. (2015) absolute $K_{S}$ band $M_{\star}$, and $T$ from NASA ExoFOP, can be constrained as follows:

$a=\sqrt[3]{\frac{G M_{\star} T^{2}}{4 \pi^{2}}}$

where $a$ is the semi-major axis, $G$ is the gravitational constant, $M_{\star}$ is the stellar mass (with $M_{\star}>>M_{p}$, the planetary mass), and $T$ is the planet orbital period - all of which we assume are independent quantities.

Now with a prior on the semi-major axis, we again use the least_squares function from scipy's optimize module to perform least squares fitting to minimise the following expression:

$R_{t}=\left(\frac{a_{R_{\star}, m}-a_{R_{\star}, f}}{\sigma_{a_{R_{\star}, m}}}+\sum_{j}^{N} \frac{t_{\mathrm{obs}, \mathrm{j}}-t_{\text {model }, \mathrm{j}}}{\sigma_{t_{\mathrm{obs}, \mathrm{j}}}}\right)^{2}$

where $R_{t}$ are the light curve and prior residuals (as a function of $R_{p, R_{\star}}, a_{R_{\star}, f}$, and $\left.i\right), a_{R_{\star}, m}$ the measured scaled semi-major axis, $a_{R_{\star}, f}$ the fitted scaled semi-major axis, $\sigma_{a_{R_{\star}, m}}$ the uncertainty on the measured scaled semi-major axis, $j$ is the time step, $N$ the total number of epochs, $t_{\mathrm{obs}, \mathrm{j}}$ is the observed flux at time step $j$, $t_{\text {model, } \mathrm{j}}$ the model flux at time step $j$, and $\sigma_{t_{\mathrm{obs}, \mathrm{j}}}$ is the measured flux uncertainty at time step $j$.

Results from this fitting procedure are presented in Table 3, a comparison with confirmed planets in Figure 11, and a histogram of the resulting planet candidate radii in Figure 12. Note that we do not fit the light curves for some candidates: TOIs 256.01 and 415969908.02 have only two and one transits respectively; TOI 507.01 is a suspected equal mass binary; TOIs 302.01 and 969.01 do not have PDCSAP two minute cadence data; and TOIs 203.01, 253.01, 285.01, 696.02, 785.01, 864.01, 1216.01, 260417932.02, and 98796344.02 have transits observed only at low SNR.

\section{DISCUSSION}

\subsection{Radial Velocities}

Just over half our TESS sample have radial velocities in Gaia DR2, with the remaining 42 therefore having an incomplete set of positional and kinematic data. Our RVs are consistent with Gaia DR2 for our overlap sample and accurate to within $\sim 4.5 \mathrm{~km} \mathrm{~s}^{-1}$ (Section 2.4), thus providing RVs for the remainder and enabling insight into Galactic population, or kinematic analysis using tools such as Chronostar (Crundall et al. 2019) to determine ages for those that are found to be members of stellar associations. These results are especially interesting given the planet-hosting nature of these stars.

\subsection{Standard Star Parameter Recovery}

Comparing our $T_{\text {eff }}$ results to those of Mann et al. (2015) reveals excellent agreement for our two parameter fit (Figure 7), with the scatter on our residuals being smaller than their mean reported uncertainty of $60 \mathrm{~K}$ and only a relatively small systematic of $\sim 30 \mathrm{~K}$ observed. Such consistency is encouraging given that this represents our largest uniform set of comparison stars, a set whose temperatures have already been successfully benchmarked against those from interferometry and should be much less sensitive to model limitations than our own.

When comparing to Rojas-Ayala et al. (2012), the results are less consistent, though we observe a similar effect to Mann et al. (2015) in that Rojas-Ayala et al. (2012) overestimates temperatures for the warmest stars. These temperatures, however, come solely from measurement of the $\mathrm{H}_{2} \mathrm{O}-\mathrm{K} 2$ index in the $K$ band in conjunction with BT-Settl model atmospheres - much more limited in wavelength coverage than Mann et al. (2015) or our work here.

The interferometric sample shows good agreement, though we observe a $\sim 70 \mathrm{~K}$ temperature systematic of the same sign as for the Mann et al. (2015) sample. However, due to the bias of interferometry towards close and thus bright targets, these are also the brightest stars we observe and they have correspondingly high photometric uncertainties due to saturation. This is particularly acute in the 2MASS bands, where less than half the sample have the photometric quality flag (Qflg) of 'AAA', in contrast to the rest of the standard sample where all but two of 117 stars has Qflg 'AAA', and the entirety of the TESS sample. Nonetheless, our derived radii for the interferometric standards (Figure 8) are consistent when allowing for additional scatter from poor quality photometry on bright stars that will not be present for our science targets. Encouragingly however Mann et al. (2015), which we are in agreement with, integrated their own photometry from low resolution flux calibrated spectra and found a good match between their results and their own interferometric sample. 
Table 3. Final results for TESS candidate exoplanets.

\begin{tabular}{|c|c|c|c|c|c|c|c|c|}
\hline TOI & TIC & Sector/s & $\begin{array}{c}\text { Period } \\
\text { (days) }\end{array}$ & $R_{p} / R_{\star}$ & $a / R_{\star}$ & $e$ flag & $\begin{array}{c}i \\
\left({ }^{\circ}\right)\end{array}$ & $\begin{array}{c}R_{p} \\
\left(R_{\oplus}\right)\end{array}$ \\
\hline 122.01 & 231702397 & $1,27-28$ & $5.07803 \dagger$ & $0.0797 \pm 0.0022$ & $24.63 \pm 0.49$ & 0 & $88.337 \pm 0.001$ & $3.00 \pm 0.10$ \\
\hline 129.01 & 201248411 & $1-2,28-29$ & $0.98097 \dagger$ & $0.3223 \pm 0.0884$ & $5.15 \pm 0.04$ & 0 & $76.381 \pm 0.018$ & $25.35 \pm 6.96$ \\
\hline 133.01 & 219338557 & 1,28 & $8.19918 \dagger$ & $0.0269 \pm 0.0010$ & $23.15 \pm 0.41$ & 0 & $88.470 \pm 0.002$ & $1.88 \pm 0.07$ \\
\hline 134.01 & 234994474 & 1,28 & $1.40153 \dagger$ & $0.0223 \pm 0.0006$ & $6.98 \pm 0.16$ & 0 & $84.566 \pm 0.005$ & $1.54 \pm 0.05$ \\
\hline 136.01 & 410153553 & $1,27-28$ & $0.46293 \dagger$ & $0.0587 \pm 0.0006$ & $6.80 \pm 0.17$ & 1 & $90.000 \pm 5.000$ & $1.23 \pm 0.03$ \\
\hline 139.01 & 62483237 & 1,28 & $11.07083 \dagger$ & $0.0346 \pm 0.0008$ & $27.20 \pm 0.56$ & 0 & $88.549 \pm 0.001$ & $2.54 \pm 0.07$ \\
\hline 142.01 & 425934411 & $1-2,28-29$ & $0.85335 \dagger$ & $0.1809 \pm 0.0184$ & $4.74 \pm 0.11$ & 0 & $79.385 \pm 0.012$ & $13.31 \pm 1.39$ \\
\hline 175.01 & 307210830 & $2,5,8-12,28-29,32$ & $3.69066 \dagger$ & $0.0397 \pm 0.0003$ & $21.32 \pm 0.47$ & 0 & $88.809 \pm 0.002$ & $1.32 \pm 0.03$ \\
\hline 175.02 & 307210830 & $2,5,8-12,28-29,32$ & $7.45075 \dagger$ & $0.0446 \pm 0.0006$ & $34.59 \pm 0.75$ & 0 & $88.483 \pm 0.001$ & $1.48 \pm 0.03$ \\
\hline 175.03 & 307210830 & $2,5,8-12,28-29,32$ & $2.25310 \dagger$ & $0.0238 \pm 0.0003$ & $15.76 \pm 0.34$ & 0 & $88.133 \pm 0.003$ & $0.79 \pm 0.02$ \\
\hline 177.01 & 262530407 & $2-3,29$ & $2.85310 \dagger$ & $0.0385 \pm 0.0005$ & $12.70 \pm 0.26$ & 0 & $86.765 \pm 0.002$ & $2.24 \pm 0.05$ \\
\hline 178.01 & 251848941 & 2,29 & $6.55770 \dagger$ & $0.0365 \pm 0.0009$ & $19.97 \pm 0.36$ & 0 & $88.506 \pm 0.002$ & $2.54 \pm 0.07$ \\
\hline 178.02 & 251848941 & 2,29 & $20.70950 \dagger$ & $0.0439 \pm 0.0023$ & $42.94 \pm 0.70$ & 0 & $88.821 \pm 0.001$ & $3.06 \pm 0.16$ \\
\hline 178.03 & 251848941 & 2,29 & $9.96188 \dagger$ & $0.0252 \pm 0.0013$ & $26.40 \pm 0.45$ & 0 & $88.855 \pm 0.002$ & $1.76 \pm 0.09$ \\
\hline $178 \mathrm{~b}$ & 251848941 & 2,29 & $1.91456 \dagger$ & $0.0197 \pm 0.0008$ & $8.78 \pm 0.15$ & 0 & $89.745 \pm 0.094$ & $1.37 \pm 0.06$ \\
\hline $178 \mathrm{c}$ & 251848941 & 2,29 & $3.23845 \dagger$ & $0.0231 \pm 0.0008$ & $12.47 \pm 0.22$ & 0 & $88.423 \pm 0.007$ & $1.61 \pm 0.06$ \\
\hline $178 \mathrm{f}$ & 251848941 & 2,29 & $15.23191 \dagger$ & $0.0314 \pm 0.0011$ & $35.01 \pm 0.59$ & 0 & $88.904 \pm 0.001$ & $2.19 \pm 0.08$ \\
\hline 198.01 & 12421862 & 2,29 & $20.43021 \dagger$ & $0.0291 \pm 0.0012$ & $58.19 \pm 1.14$ & 0 & $89.374 \pm 0.001$ & $1.31 \pm 0.06$ \\
\hline 210.01 & 141608198 & $1-5,7-13,27-32$ & $9.01056 \dagger$ & $0.0629 \pm 0.0007$ & $37.78 \pm 0.66$ & 0 & $89.531 \pm 0.002$ & $2.24 \pm 0.05$ \\
\hline 233.01 & 415969908 & 2,29 & $11.66993 \dagger$ & $0.0457 \pm 0.0013$ & $42.65 \pm 0.82$ & 0 & $89.606 \pm 0.002$ & $1.82 \pm 0.06$ \\
\hline 234.01 & 12423815 & 2,29 & $2.83927 \dagger$ & $0.1932 \pm 0.0045$ & $12.63 \pm 0.28$ & 0 & $86.641 \pm 0.003$ & $11.50 \pm 0.39$ \\
\hline 240.01 & 101948569 & 2,29 & $19.47241 \dagger$ & $0.0388 \pm 0.0013$ & $38.59 \pm 0.59$ & 0 & $89.278 \pm 0.001$ & $2.93 \pm 0.10$ \\
\hline 244.01 & 118327550 & 2,29 & $7.39719 \dagger$ & $0.0321 \pm 0.0012$ & $29.05 \pm 0.56$ & 0 & $88.382 \pm 0.001$ & $1.42 \pm 0.06$ \\
\hline 249.01 & 179985715 & 2,29 & $6.61542 \dagger$ & $0.0329 \pm 0.0018$ & $22.18 \pm 0.38$ & 0 & $88.757 \pm 0.003$ & $2.01 \pm 0.11$ \\
\hline 256.02 & 92226327 & 3,30 & $3.77796 \dagger$ & $0.0480 \pm 0.0010$ & $25.61 \pm 0.59$ & 0 & $90.000 \pm 5.000$ & $1.15 \pm 0.03$ \\
\hline 260.01 & 37749396 & 3 & 13.470018 & $0.0265 \pm 0.0010$ & $34.87 \pm 0.60$ & 0 & $88.758 \pm 0.001$ & $1.66 \pm 0.07$ \\
\hline 269.01 & 220479565 & $3-6,10,13,30-32$ & $3.69770 \dagger$ & $0.0691 \pm 0.0012$ & $18.65 \pm 0.31$ & 0 & $87.384 \pm 0.001$ & $2.99 \pm 0.07$ \\
\hline 270.01 & 259377017 & $3-5,30,32$ & $5.66054 \dagger$ & $0.0581 \pm 0.0004$ & $26.06 \pm 0.55$ & 0 & $89.210 \pm 0.002$ & $2.29 \pm 0.04$ \\
\hline 270.02 & 259377017 & $3-5,30,32$ & $11.37960 \dagger$ & $0.0535 \pm 0.0004$ & $41.87 \pm 0.78$ & 0 & $89.707 \pm 0.002$ & $2.11 \pm 0.04$ \\
\hline 270.03 & 259377017 & $3-5,30,32$ & $3.36014 \dagger$ & $0.0301 \pm 0.0005$ & $18.65 \pm 0.37$ & 0 & $89.218 \pm 0.005$ & $1.18 \pm 0.03$ \\
\hline 279.01 & 122613513 & $3-4$ & 11.494122 & $0.0361 \pm 0.0013$ & $27.95 \pm 0.45$ & 0 & $88.583 \pm 0.001$ & $2.65 \pm 0.10$ \\
\hline 406.01 & 153065527 & $3-4,30-31$ & $13.17573 \dagger$ & $0.0430 \pm 0.0013$ & $43.35 \pm 0.87$ & 0 & $89.303 \pm 0.001$ & $1.84 \pm 0.06$ \\
\hline 435.01 & 44647437 & $4-5,31$ & $3.35293 \dagger$ & $0.0583 \pm 0.0016$ & $12.82 \pm 0.23$ & 0 & $88.622 \pm 0.007$ & $3.99 \pm 0.12$ \\
\hline 442.01 & 70899085 & 5,32 & $4.05203 \dagger$ & $0.0741 \pm 0.0007$ & $14.48 \pm 0.25$ & 0 & $86.865 \pm 0.002$ & $5.02 \pm 0.09$ \\
\hline 455.01 & 98796344 & 4,31 & $5.35880 \dagger$ & $0.0467 \pm 0.0009$ & $29.82 \pm 1.22$ & 0 & $89.391 \pm 0.005$ & $1.38 \pm 0.04$ \\
\hline 468.01 & 33521996 & 6,32 & $3.32527 \dagger$ & $0.1735 \pm 0.0014$ & $12.63 \pm 0.25$ & 0 & $87.382 \pm 0.003$ & $12.01 \pm 0.24$ \\
\hline 475.01 & 100608026 & $5-6,32$ & $8.26159 \dagger$ & $0.0307 \pm 0.0014$ & $24.22 \pm 0.42$ & 0 & $89.006 \pm 0.003$ & $2.00 \pm 0.10$ \\
\hline 486.01 & 260708537 & $1-6,8-13,27-32$ & $1.74468 \dagger$ & $0.0128 \pm 0.0003$ & $10.82 \pm 0.21$ & 0 & $88.585 \pm 0.008$ & $0.59 \pm 0.02$ \\
\hline 493.01 & 19025965 & 7 & 5.947773 & $0.0518 \pm 0.0020$ & $17.60 \pm 0.25$ & 0 & $87.987 \pm 0.003$ & $3.90 \pm 0.16$ \\
\hline 521.01 & 27649847 & 7 & 1.542131 & $0.0463 \pm 0.0028$ & $9.89 \pm 0.17$ & 0 & $86.478 \pm 0.006$ & $2.15 \pm 0.14$ \\
\hline 532.01 & 144700903 & 6 & 2.326811 & $0.0876 \pm 0.0020$ & $9.96 \pm 0.14$ & 0 & $87.050 \pm 0.004$ & $6.02 \pm 0.17$ \\
\hline 540.01 & 200322593 & $4-6,31-32$ & $1.23914 \dagger$ & $0.0366 \pm 0.0010$ & $13.48 \pm 0.32$ & 0 & $87.063 \pm 0.003$ & $0.78 \pm 0.03$ \\
\hline 544.01 & 50618703 & 6,32 & $1.54835 \dagger$ & $0.0281 \pm 0.0006$ & $7.80 \pm 0.14$ & 0 & $85.103 \pm 0.004$ & $1.91 \pm 0.05$ \\
\hline 551.01 & 192826603 & $5-6,32$ & $2.64730 \dagger$ & $0.3387 \pm 1.1540$ & $11.60 \pm 0.17$ & 0 & $84.411 \pm 0.118$ & $21.32 \pm 72.62$ \\
\hline 552.01 & 44737596 & $4-5,31$ & $2.78864 \dagger$ & $0.1552 \pm 0.0014$ & $11.57 \pm 0.19$ & 0 & $87.675 \pm 0.003$ & $10.05 \pm 0.20$ \\
\hline 555.01 & 170849515 & $5,31-32$ & $1.94163 \dagger$ & $0.1548 \pm 0.0028$ & $8.92 \pm 0.20$ & 0 & $87.380 \pm 0.008$ & $10.57 \pm 0.32$ \\
\hline 557.01 & 55488511 & 5,31 & $3.34499 \dagger$ & $0.0388 \pm 0.0025$ & $13.43 \pm 0.21$ & 0 & $86.264 \pm 0.002$ & $2.46 \pm 0.16$ \\
\hline 562.01 & 413248763 & 8 & 3.930792 & $0.0329 \pm 0.0007$ & $20.86 \pm 0.41$ & 0 & $88.691 \pm 0.002$ & $1.27 \pm 0.03$ \\
\hline 620.01 & 296739893 & 8 & 5.098373 & $0.0597 \pm 0.0015$ & $18.65 \pm 0.35$ & 0 & $87.394 \pm 0.001$ & $3.56 \pm 0.11$ \\
\hline 654.01 & 35009898 & 9 & 1.527419 & $0.0513 \pm 0.0019$ & $9.44 \pm 0.16$ & 0 & $87.873 \pm 0.010$ & $2.49 \pm 0.10$ \\
\hline 656.01 & 36734222 & 9 & 0.813470 & $0.1608 \pm 0.0005$ & $4.67 \pm 0.03$ & 0 & $81.396 \pm 0.002$ & $12.11 \pm 0.19$ \\
\hline 663.01 & 54962195 & 9 & 2.598654 & $0.0408 \pm 0.0017$ & $12.24 \pm 0.17$ & 0 & $88.685 \pm 0.010$ & $2.32 \pm 0.11$ \\
\hline 663.02 & 54962195 & 9 & 4.698465 & $0.0433 \pm 0.0023$ & $18.16 \pm 0.25$ & 0 & $88.488 \pm 0.004$ & $2.46 \pm 0.14$ \\
\hline 672.01 & 151825527 & $9-10$ & 3.633618 & $0.0889 \pm 0.0008$ & $14.44 \pm 0.20$ & 0 & $87.932 \pm 0.002$ & $5.45 \pm 0.10$ \\
\hline 674.01 & 158588995 & $9-10$ & 1.977238 & $0.1174 \pm 0.0009$ & $11.35 \pm 0.17$ & 0 & $86.352 \pm 0.002$ & $5.67 \pm 0.11$ \\
\hline 696.01 & 77156829 & $4-5,31-32$ & $0.86024 \dagger$ & $0.0221 \pm 0.0008$ & $7.96 \pm 0.16$ & 0 & $84.721 \pm 0.004$ & $0.79 \pm 0.03$ \\
\hline 698.01 & 141527579 & $1-5,7-13,27-32$ & $15.08666 \dagger$ & $0.0436 \pm 0.0010$ & $42.17 \pm 0.68$ & 0 & $89.058 \pm 0.001$ & $2.26 \pm 0.07$ \\
\hline 700.01 & 150428135 & $1,3-11,13,27-28,30-31$ & $16.05110 \dagger$ & $0.0573 \pm 0.0010$ & $47.21 \pm 0.89$ & 0 & $88.902 \pm 0.000$ & $2.66 \pm 0.07$ \\
\hline 700.02 & 150428135 & $1,3-11,13,27-28,30-31$ & $37.42475 \dagger$ & $0.0272 \pm 0.0008$ & $82.17 \pm 1.66$ & 0 & $90.000 \pm 5.307$ & $1.26 \pm 0.04$ \\
\hline 700.03 & 150428135 & $1,3-11,13,27-28,30-31$ & $9.97701 \dagger$ & $0.0181 \pm 0.0009$ & $34.16 \pm 0.66$ & 0 & $89.885 \pm 0.014$ & $0.84 \pm 0.04$ \\
\hline
\end{tabular}

Notes: Periods denoted by $\dagger$ are not as reported by ExoFOP, and have been refitted here. These are overwhelmingly systems with TESS extended mission data, thus having longer time baselines with which to constrain orbital periods. Our fitted periods however are generally consistent within uncertainties of their ExoFOP values, and as such we do not report new uncertainties here. Additionally, our least squares fits to 7 of our light curves proved insenstive to non-edge-on inclinations. As such, we report conservative uncertanties of $\pm 5^{\circ}$ for these planets. 
Table 3 - continued Final results for TESS candidate exoplanets

\begin{tabular}{|c|c|c|c|c|c|c|c|c|}
\hline TOI & TIC & Sector/s & $\begin{array}{l}\begin{array}{l}\text { Period } \\
\text { (days) }\end{array} \\
\end{array}$ & $R_{p} / R_{\star}$ & $a / R_{\star}$ & $e$ flag & $\begin{array}{c}i \\
\left({ }^{\circ}\right) \\
\end{array}$ & $\begin{array}{c}R_{p} \\
\left(R_{\oplus}\right)\end{array}$ \\
\hline 702.01 & 237914496 & $1-4,7,11,27,29-31$ & $3.56809 \dagger$ & $0.0280 \pm 0.0010$ & $14.46 \pm 0.24$ & 0 & $87.421 \pm 0.002$ & $1.73 \pm 0.07$ \\
\hline 704.01 & 260004324 & $1-5,7-13,27-32$ & $3.81431 \dagger$ & $0.0208 \pm 0.0004$ & $15.38 \pm 0.28$ & 0 & $87.419 \pm 0.002$ & $1.21 \pm 0.03$ \\
\hline 711.01 & 38510224 & $1-5,7-8,11-12,28-32$ & $18.38384 \dagger$ & $0.0301 \pm 0.0012$ & $39.90 \pm 0.64$ & 0 & $89.221 \pm 0.001$ & $2.09 \pm 0.09$ \\
\hline 713.01 & 167600516 & $1-8,10-13,27-32$ & $35.99988 \dagger$ & $0.0315 \pm 0.0006$ & $62.37 \pm 0.95$ & 0 & $89.724 \pm 0.001$ & $2.19 \pm 0.05$ \\
\hline 713.02 & 167600516 & $1-8,10-13,27-32$ & $1.87150 \dagger$ & $0.0155 \pm 0.0007$ & $8.69 \pm 0.13$ & 0 & $84.872 \pm 0.003$ & $1.07 \pm 0.05$ \\
\hline 714.01 & 219195044 & $4-8,11-12,28,31-32$ & $4.32378 \dagger$ & $0.0260 \pm 0.0010$ & $18.59 \pm 0.31$ & 0 & $88.556 \pm 0.003$ & $1.33 \pm 0.06$ \\
\hline 714.02 & 219195044 & $4-8,11-12,28,31-32$ & $10.17742 \dagger$ & $0.0307 \pm 0.0011$ & $32.90 \pm 0.51$ & 0 & $89.108 \pm 0.001$ & $1.57 \pm 0.06$ \\
\hline 727.01 & 149788158 & 8 & 4.726090 & $0.0293 \pm 0.0020$ & $18.76 \pm 0.31$ & 0 & $89.435 \pm 0.014$ & $1.60 \pm 0.11$ \\
\hline 731.01 & 34068865 & 9 & 0.321941 & $0.0133 \pm 0.0005$ & $3.29 \pm 0.07$ & 0 & $85.081 \pm 0.031$ & $0.67 \pm 0.03$ \\
\hline 732.01 & 36724087 & 9 & 0.768418 & $0.0311 \pm 0.0011$ & $6.59 \pm 0.13$ & 0 & $90.000 \pm 5.000$ & $1.30 \pm 0.05$ \\
\hline 732.02 & 36724087 & 9 & 12.254218 & $0.0607 \pm 0.0022$ & $41.84 \pm 0.74$ & 0 & $88.868 \pm 0.001$ & $2.53 \pm 0.10$ \\
\hline 741.01 & 359271092 & $9-10$ & 7.576262 & $0.0160 \pm 0.0009$ & $25.25 \pm 0.62$ & 0 & $88.472 \pm 0.002$ & $0.91 \pm 0.05$ \\
\hline 756.01 & 73649615 & $10-11$ & $1.23952 \dagger$ & $0.0548 \pm 0.0019$ & $7.43 \pm 0.10$ & 0 & $85.039 \pm 0.005$ & $3.12 \pm 0.12$ \\
\hline 761.01 & 165317334 & 10 & 10.563348 & $0.0417 \pm 0.0016$ & $27.53 \pm 0.41$ & 0 & $89.196 \pm 0.003$ & $2.86 \pm 0.12$ \\
\hline 782.01 & 429358906 & 10 & 16.047203 & $0.0659 \pm 0.0040$ & $47.81 \pm 0.69$ & 0 & $89.070 \pm 0.001$ & $2.97 \pm 0.19$ \\
\hline 789.01 & 300710077 & $1-3,5-13,27-32$ & $5.44693 \dagger$ & $0.0274 \pm 0.0011$ & $24.99 \pm 0.44$ & 0 & $89.127 \pm 0.003$ & $1.11 \pm 0.05$ \\
\hline 797.01 & 271596225 & $1-13,27-32$ & $1.80078 \dagger$ & $0.0256 \pm 0.0006$ & $10.21 \pm 0.17$ & 0 & $86.580 \pm 0.003$ & $1.33 \pm 0.04$ \\
\hline 797.02 & 271596225 & $1-13,27-32$ & $4.14002 \dagger$ & $0.0292 \pm 0.0011$ & $17.78 \pm 0.30$ & 0 & $87.236 \pm 0.001$ & $1.52 \pm 0.06$ \\
\hline 806.01 & 33831980 & $1-3,5-6,8-9,12-13,27-29,32$ & $21.91625 \dagger$ & $0.0347 \pm 0.0011$ & $47.14 \pm 0.66$ & 0 & $89.479 \pm 0.001$ & $2.24 \pm 0.08$ \\
\hline 824.01 & 193641523 & $11-12$ & 1.392930 & $0.0436 \pm 0.0007$ & $6.69 \pm 0.10$ & 0 & $83.665 \pm 0.004$ & $3.29 \pm 0.07$ \\
\hline 833.01 & 362249359 & $9-11$ & 1.042241 & $0.0190 \pm 0.0007$ & $6.02 \pm 0.10$ & 0 & $89.977 \pm 1.790$ & $1.24 \pm 0.05$ \\
\hline 836.01 & 440887364 & 11 & 8.593935 & $0.0346 \pm 0.0006$ & $23.32 \pm 0.30$ & 0 & $88.727 \pm 0.001$ & $2.49 \pm 0.06$ \\
\hline 836.02 & 440887364 & 11 & 3.817115 & $0.0240 \pm 0.0008$ & $13.59 \pm 0.23$ & 0 & $87.727 \pm 0.003$ & $1.72 \pm 0.06$ \\
\hline 870.01 & 219229644 & $3-5,30-32$ & $22.03813 \dagger$ & $0.0330 \pm 0.0012$ & $45.25 \pm 0.81$ & 0 & $89.013 \pm 0.001$ & $2.22 \pm 0.09$ \\
\hline 873.01 & 237920046 & $1-4,11,28-31$ & $5.93122 \dagger$ & $0.0279 \pm 0.0012$ & $21.26 \pm 0.38$ & 0 & $90.000 \pm 5.000$ & $1.58 \pm 0.07$ \\
\hline 875.01 & 14165625 & $5-6$ & 11.020153 & $0.0277 \pm 0.0021$ & $26.47 \pm 0.36$ & 0 & $90.000 \pm 5.000$ & $2.08 \pm 0.16$ \\
\hline 876.01 & 32497972 & $5-6,32$ & $38.69629 \dagger$ & $0.0367 \pm 0.0044$ & $65.85 \pm 1.39$ & 0 & $89.254 \pm 0.001$ & $2.46 \pm 0.30$ \\
\hline 900.01 & 210873792 & 12 & 4.844050 & $0.0426 \pm 0.0026$ & $15.81 \pm 0.26$ & 0 & $90.000 \pm 5.000$ & $3.01 \pm 0.19$ \\
\hline 904.01 & 261257684 & $12-13$ & $18.35654 \dagger$ & $0.0342 \pm 0.0017$ & $43.55 \pm 0.79$ & 0 & $90.000 \pm 5.000$ & $2.00 \pm 0.10$ \\
\hline 910.01 & 369327947 & $12-13,27$ & $2.02911 \dagger$ & $0.0311 \pm 0.0007$ & $15.53 \pm 0.31$ & 0 & $87.229 \pm 0.002$ & $0.94 \pm 0.03$ \\
\hline 912.01 & 406941612 & $12-13$ & 4.679100 & $0.0414 \pm 0.0008$ & $20.71 \pm 0.32$ & 0 & $88.819 \pm 0.002$ & $1.93 \pm 0.05$ \\
\hline 929.01 & 175532955 & $30-31$ & $5.83010 \dagger$ & $0.0314 \pm 0.0015$ & $17.32 \pm 0.28$ & 0 & $89.396 \pm 0.012$ & $2.37 \pm 0.12$ \\
\hline 932.01 & 260417932 & $28-29,31-32$ & 19.310700 & $0.0337 \pm 0.0009$ & $38.00 \pm 0.62$ & 0 & $89.463 \pm 0.002$ & $2.59 \pm 0.08$ \\
\hline 1067.01 & 201642601 & 13,27 & $3.13167 \dagger$ & $0.1071 \pm 0.0012$ & $11.13 \pm 0.20$ & 0 & $89.174 \pm 0.012$ & $8.42 \pm 0.17$ \\
\hline 1073.01 & 158297421 & 13,27 & $3.92282 \dagger$ & $0.1799 \pm 0.0039$ & $13.88 \pm 0.27$ & 0 & $86.903 \pm 0.002$ & $13.04 \pm 0.39$ \\
\hline 1075.01 & 351601843 & 13,27 & $0.60474 \dagger$ & $0.0286 \pm 0.0006$ & $4.39 \pm 0.08$ & 0 & $85.023 \pm 0.014$ & $1.78 \pm 0.05$ \\
\hline 1078.01 & 370133522 & 13,27 & $0.51824 \dagger$ & $0.0274 \pm 0.0004$ & $5.04 \pm 0.10$ & 0 & $85.016 \pm 0.010$ & $1.17 \pm 0.03$ \\
\hline 1082.01 & 261108236 & $12-13,27-28,31$ & $16.34646 \dagger$ & $0.0399 \pm 0.0012$ & $37.72 \pm 0.63$ & 0 & $89.448 \pm 0.002$ & $2.65 \pm 0.09$ \\
\hline 1201.01 & 29960110 & 4,31 & $2.49197 \dagger$ & $0.0389 \pm 0.0009$ & $12.46 \pm 0.24$ & 0 & $87.967 \pm 0.004$ & $2.07 \pm 0.06$ \\
\hline 153065527.02 & 153065527 & $3-4,30-31$ & $3.30745 \dagger$ & $0.0270 \pm 0.0013$ & $17.26 \pm 0.33$ & 0 & $87.940 \pm 0.003$ & $1.16 \pm 0.06$ \\
\hline
\end{tabular}

Finally, our results are consistent with our sample of mid-Kdwarfs in the temperature range of our warmest science targets. The observed higher scatter (than e.g. the Mann et al. 2015 sample) is to be expected due to inter-study systematics, as these targets were not pulled from a single uniform catalogue.

While the exact cause of the Mann et al. (2015) and interferometric systematic is unclear, its appearance in both samples suggests it is not an artefact. As such, we apply a $-30 \mathrm{~K}$ correction to the observed temperature systematic. Although our remaining scatter is consistent with the scatter in our external reference catalogues, we add a further $\pm 30 \mathrm{~K} T_{\text {eff }}$ uncertainty in quadrature with our statistical uncertainties to account for the unknown origin of the observed systematic. Given these corrections, we are confident our fitting methodology is able to recover both accurate and precise stellar temperatures and radii for stars in the range $3000 \mathrm{~K} \lesssim T_{\text {eff }} \lesssim 4500 \mathrm{~K}$ - critical for insight into the radii of their transiting planets.

\subsection{Model Limitations}

The inability of cool dwarf atmospheric models to reproduce optical fluxes is significant. Such wavelengths are among the most easily accessible, and understanding them is required to take full advantage of photometry from surveys like Gaia and SkyMapper. Thus anyone relying directly (e.g. spectral fitting) or indirectly (e.g. isochrone fitting with colours) on models for cool stellar atmospheres must do so with caution (for specifics on isochone systematics, see e.g. VandenBerg et al. 2006 for the Victoria-Regina models, Dotter et al. 2008 and Joyce \& Chaboyer 2018 for DSEP, and Dotter 2016 for MIST).

We identify two key areas for improvement with our models and methods as implemented. The first relates to TiO, the dominant opacity source at optical wavelengths. Comparing high-resolution spectra of M-dwarfs to PHOENIX models and TiO templates, Hoeijmakers et al. (2015) concluded that 'the modelled spectrum of TiO is not representative of the real TiO'. McKemmish et al. (2019) confirmed this discrepancy in the process of validating their updated TiO line list, with their comparisons showing significant improvements in both predicted $\mathrm{TiO}$ wavelengths and line depths across the 
optical when using the updated line list. McKemmish et al. (2019) was not yet published at the time our MARCS models were generated, and although they note that there remains room for further work, this represents a significant improvement on the previous state of the art. While recomputing our library of synthetic spectra with the new line list would constitute a significant computational effort, we will endeavour to do this in future work.

The second issue concerns proper consideration of the relative abundances of $\mathrm{C}$ and $\mathrm{O}$ - constituents of the dominant molecular opacity sources in cool dwarf atmospheres-denoted here as [(OC)/Fe]. As described by Veyette et al. (2016), it is not just $T_{\text {eff }}$ and $[\mathrm{Fe} / \mathrm{H}]$ that affects the location of the pseudo-continuum, but also $[(\mathrm{O}-\mathrm{C}) / \mathrm{Fe}]$. The principal reason for this is that $[(\mathrm{O}-\mathrm{C}) / \mathrm{Fe}]$ influences the concentrations of $\mathrm{C}$ - and $\mathrm{O}$-based molecules, affecting the flux of the pseudo-continuum and apparent strength of metal lines. They conclude that ultimately the inferred value of $[\mathrm{Fe} / \mathrm{H}]$ depends on $[(\mathrm{O}-\mathrm{C}) / \mathrm{Fe}]$, and that much better spectral fits are possible when allowing $[(\mathrm{O}-\mathrm{C}) / \mathrm{Fe}]$ to vary. An important note is that empirical calibrations based on FGK-M binaries such as Rojas-Ayala et al. (2012), Mann et al. (2013a), and our own photometric [Fe/H] relation should remain valid as statistical $[\mathrm{Fe} / \mathrm{H}]$ indicators due to the tight Solar Neighbourhood $[\mathrm{Fe} / \mathrm{H}]-[\mathrm{C} / \mathrm{O}]$ correlation. Per the recommendation of Veyette et al. (2016), this issue is significant enough to merit new models with $[\mathrm{C} / \mathrm{Fe}]$ and $[\mathrm{O} / \mathrm{Fe}]$ as independent parameters.

That said, there is an ever increasing empirical knowledge of M-dwarfs meaning that, even in the absence of accurate models, empirical or data driven approaches should be possible, especially if methods to break the $[\mathrm{Fe} / \mathrm{H}]-[(\mathrm{O}-\mathrm{C}) / \mathrm{Fe}]$ degeneracy can be found. For instance, see Birky et al. (2020) which demonstrates that a data driven approach, at least in the $H$ band, is possible for M-dwarfs. The very small rate of evolution for these low-mass stars means we can rely on mass and chemical composition to derive the fundamental parameters of the star, thus making for a more tractable problem.

\subsection{TESS Input Catalogue Stellar Parameters}

The TESS Input Catalogue is often the first stellar parameter reference for newly alerted TOIs. As these parameters are mostly derived from empirical relations using literature photometry, we thought it useful to compare these predictions with our fits to inspect for remaining catalogue systematics. Figure 9 displays this comparison for $T_{\text {eff }}$ and $R_{\star}$, and while the TIC temperatures are broadly consistent, TIC radii for the warmest stars in our sample appear systematically large. This stellar radii systematic is noteworthy as it would bias any predicted exoplanet radii around mid-K dwarfs.

\subsection{Emission Features in TESS Candidates}

While model limitations prevented us from taking full advantage of our spectra during fitting, our wide wavelength coverage allows us to look for spectral peculiarities. In the current study, these take the form of emission in the Hydrogen Balmer Series or Ca II H\&K (both signs of stellar activity and youth), as well as absorption in the Li 6, $708 \AA$ (another sign of youth). While none of our TESS planet hosts show detectable Lithium absorption, we report $\mathrm{H} \alpha$ equivalent widths and $\log R_{\mathrm{HK}}^{\prime}$ in Table 2, calculated using the methodology of Žerjal et al. (2021). 53 stars in our sample have EWH $\alpha>-0.5 \AA$ (adopted as the limiting bound for activity, noting as well that this is strongly dependent on $T_{\text {eff }}$ and thus somewhat approximate), and
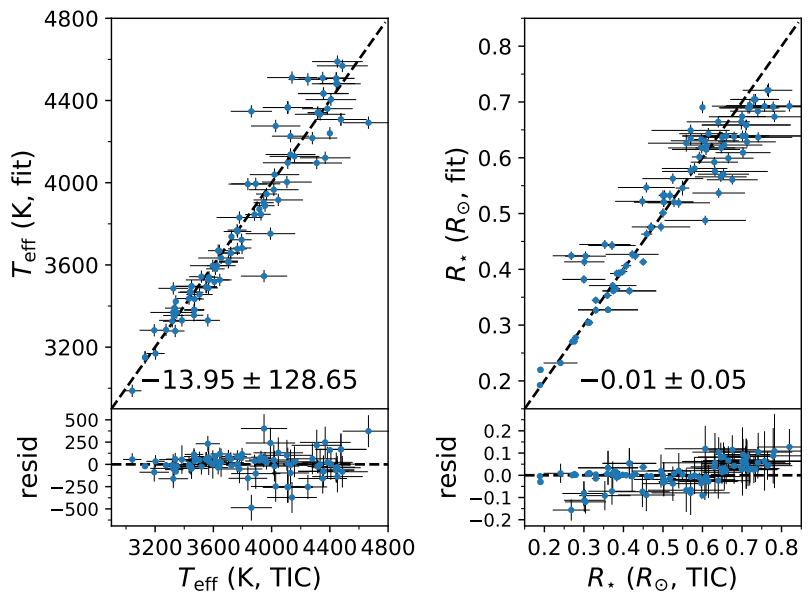

Figure 9. Comparison of $T_{\mathrm{eff}}$ and $R_{\star}$ as reported here compared to those from the TESS Input Catalogue. The median and standard deviation of each set of residuals is annotated.

35 have $\log R_{\mathrm{HK}}^{\prime}>-4.75$ (the lower bound for active stars used in Gray et al. 2006).

Of particular note are our two most active stars, the first of which is TOI 507 (TIC 348538431). TOI 507 appears substantially overluminous in Figure 1, and presents with strong emission across the Balmer Series and in Ca II H\&K. Visual inspection of its spectrum, along with comparison to the cool dwarf standard HIP 103039 which is very similar in $T_{\text {eff }}$, indicates that it is actually a doublelined spectroscopic binary. Transit depths appear similar for both primary and secondary eclipses, which points to the system being composed of roughly equal mass components. Taking a $\sim 0.75 \mathrm{mag}$ offset into account due to binarity, TOI 507 still sits slightly above the main sequence, meaning that it remains a potentially young touchstone system amenable to characterisation as in e.g. Murphy et al. (2020). The mass, radius, $m_{\text {bol }}$, and flux reported in Table 2 have been derived for a single component of this binary system, assuming equal mass and brightness.

The second star is TOI 142 (425934411) which is also overluminous and shows displays strong emission features. Interestingly, it appears to host a giant planet $\left(R_{P}=13.31 \pm 1.39 R_{\oplus}\right)$ on a short period ( $T \approx 0.85$ day) - see Figure 10 . While this is unusual for such a cool star, it is not unheard of, such as K2 $32 \mathrm{~b}$ which is a known short period super-Neptune orbiting a pre-main sequence star (David et al. 2016; Mann et al. 2016). Further characterisation of the system however, whilst scientifically interesting, is likely to be hampered by the faintness of the host star $(G \sim 15.8)$.

\subsection{Planet Parameter Recovery}

Table E1 collates literature parameters for previously characterised planets in our sample. These planets have typically had followup radial velocity observations which not only allows for planetary mass determination, but helps constrain their orbits when combined with the TESS light curves we use here (or additional time series photometric follow up). Figure 11 compares these results to our own for $R_{P} / R_{\star}, a / R_{\star}, i$, and $R_{P}$. We find our results consistent with the literature, aside from a few exceptions discussed below. 


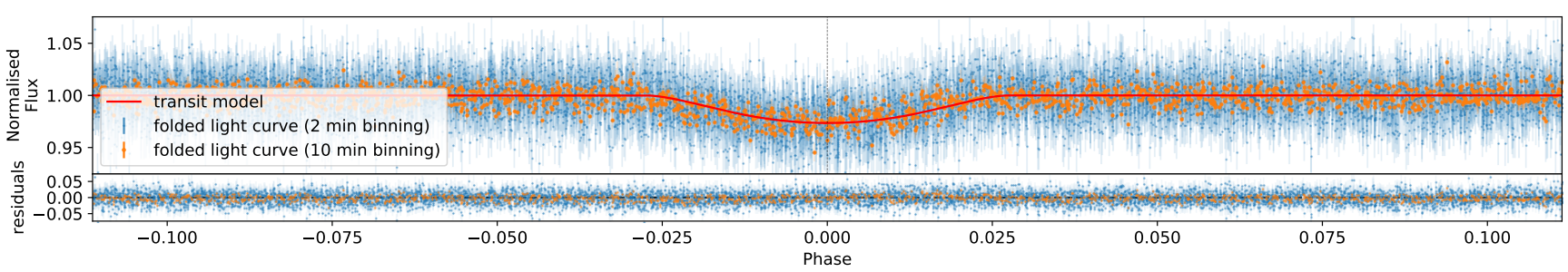

Figure 10. Phase folded light curve with best fitting transit model for TOI 142.01.

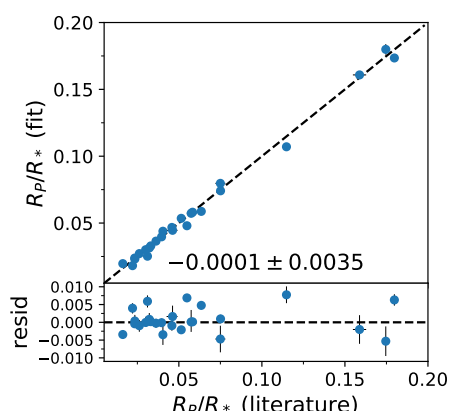

$R_{P} / R_{*}$ (literature)
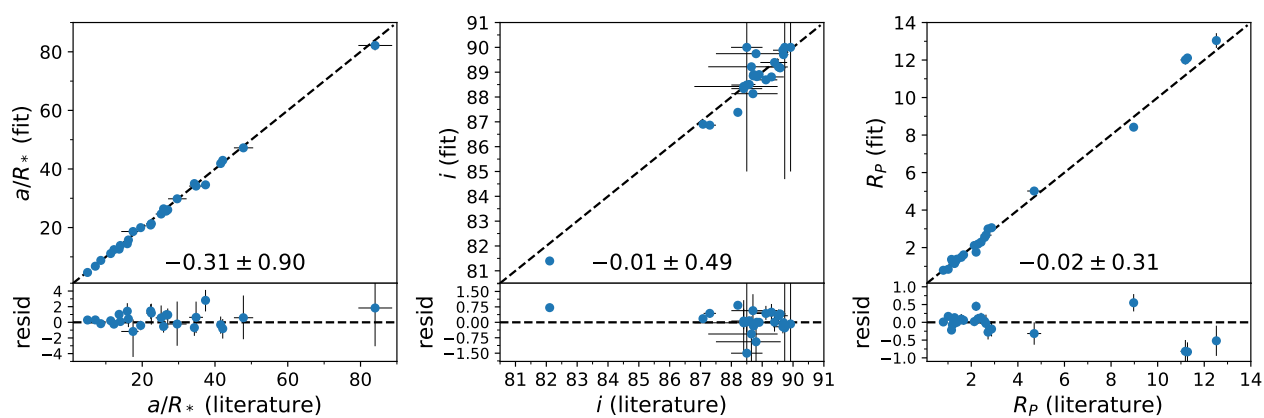

Figure 11. Comparison of $R_{P} / R_{\star}, a / R_{\star}, i$, and $R_{P}$ to literature results in Table E1. Our two largest literature planets, TOIs 129.01 and 551.01, are hot Jupiters in a grazing configuration which leaves their radii poorly constrained. As such, they have been left off for clarity, though our results are consistent within uncertainties. The median and standard deviation of each set of residuals is annotated and excludes these two planets.

\subsubsection{LHS $3844 b$}

Vanderspek et al. (2019) reports a larger value of $R p / R_{\star}$ for LHS $3844 \mathrm{~b}$ (TOI 136.01) than we do here, a difference we can attribute to our access to an extra sector of TESS data. While they also have ground based data, the extra TESS sector amounts to some 60 extra transits, which should give us improved precision.

\subsubsection{HATS-48 A b, TOI 178 b/e, LHS $1140 c$}

Comparison with HATS-48 A b (TOI 1067.01) from Hartman et al. (2020) shows an inconsistent value of $R_{P} / R_{\star}$, indicating a difference in how we have modelled the light curves. While we have access to an additional sector of TESS data, the difference primarily appears to come from a) including RVs in their fit, and b) their use of an additional 'dilution factor' when fitting to account for nearby unresolved stars. Such nearby stars have the effect of diluting the transit and making the transit appear shallower than it would were only the flux from the host star observed. Our transit fits, by comparison, rely on the quality of the detrending and correction for crowding already done by the TESS team and provided in their PDCSAP fluxes.

Leleu et al. (2021) reports parameters for six planets orbiting TOI 178, of which only three were alerted on as TOIs. Our parameters are consistent for all but two of these, TOI $178 \mathrm{~b}$ (not alerted on) and TOI 178.03, both of which are relatively low SNR detections by TESS. Although our analysis includes an additional TESS sector of data, they employ higher precision data from CHEOPS to which we attribute the difference.

The analysis of LHS $1140 \mathrm{c}$ (TOI 256.02) by Ment et al. (2019) results in a value of $R_{P} / R_{\star}$ discrepant with our own. While our analysis makes use of an additional sector of TESS data, we consider their results more reliable as they conducted a joint RV and transit photometry analysis, including additional ground based data alongside high precision Spitzer data.

\subsubsection{WASP-43 $b$ and HATS-6 $b$}

We find a consistent $R_{P} / R_{\star}$ with Esposito et al. (2017) for WASP$43 \mathrm{~b}$ (TOI 656.01), though our value of $R_{P}$ is smaller. This difference is attributable to their larger and less precise stellar $T_{\text {eff }}$, with which they obtain a smaller stellar radius - resulting in a smaller planetary radii. As discussed, we are confident with our $T_{\text {eff }}$ and $R_{\star}$ recovery, and consider the difference the result of differing approaches to stellar parameter determination.

For HATS-6 b (TOI 468.01) we find our $R_{P} / R_{\star}$ and $T_{\text {eff }}$ consistent, but a different value for $R_{P}$ as compared to Hartman et al. (2015). This difference again arises from a smaller literature value of $R_{\star}$. We consider our approach to radius determination using stellar fluxes more direct than the modelling based approach used here, especially given our access to precision Gaia parallaxes.

\subsection{Candidate Planet Radii Distribution}

We plot a histogram of our candidate planet radii in Figure 12, which shows the existence of the planet radius gap, first identified by Fulton et al. (2017), at $\sim 1.65-2.0 R_{\oplus}$ at a $\sim 1 \sigma$ level. As we remain limited by our small sample size, we do not perform any additional analysis and leave such investigations for future studies based on a larger sample of TESS planets.

Our results however do provide encouraging further evidence for the radius gap being present around planets orbiting low-mass 


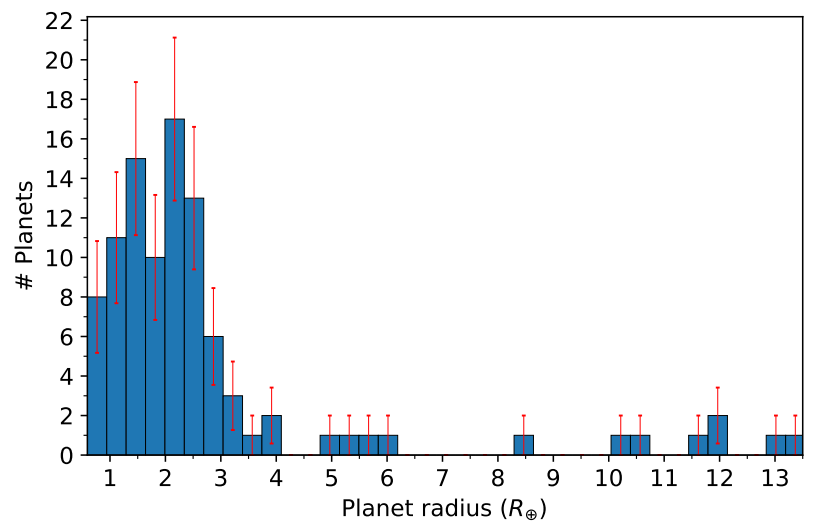

Figure 12. Histogram of candidate planet radii with $R_{P}<14 R_{\oplus}$, with $0.35 R_{\oplus}$ width bins and Poisson uncertainties. Note that we detect the exoplanet radius gap at approximately a $\sim 1 \sigma$ level, though remain limited by our small sample size.

stars. Its detection for the stellar mass range considered here is similar to the work of Cloutier \& Menou (2020) who investigated a set of confirmed and candidate planets from Kepler and K2 orbiting stars with $T_{\mathrm{eff}}<4,700 \mathrm{~K}$, with their sample being roughly a factor of $\sim 4.5$ larger than our own. Separating their planets into bins of different stellar mass, they demonstrated that the bimodality in the radius distribution vanishes as stellar mass decreases, corresponding to the population of rocky planets beginning to dominate that of their more gas rich counterparts. They note, however, that a much larger sample of planets is required in order to properly distinguish between the various possible formation channels for the radius valley (e.g. photoevaporation, core-powered mass loss), particularly when further subdividing the sample by stellar mass. It is hoped that our results here can contribute to a larger future analysis combining Kepler, $\mathrm{K} 2$, and TESS planets, perhaps also looking into correlations with stellar activity using activity measures such as we provide.

\section{CONCLUSIONS}

In the work presented above, we have described our ANU $2.3 \mathrm{~m} / \mathrm{WiFeS}$ observing program to characterise 92 southern TESS candidate planet hosts with $3,000 \lesssim T_{\text {eff }} \lesssim 4,500 \mathrm{~K}$ in order to precisely determine the radii of 100 transiting planets they host. In the process of doing so we investigated cool dwarf model atmosphere systematics, as well as developed a new photometric $[\mathrm{Fe} / \mathrm{H}]$ calibration. The main conclusions from our work are as follows:

- Cool dwarf MARCS model atmospheres systematically overestimate flux in the optical relative to the well produced spectral regions 5585-6029 $\AA$ and 6159-7000 $\mathrm{A}$, with agreement being worse the cooler the star or bluer the wavelength. We report a simple linear relation parameterising the offset as a function of the observed Gaia $\left(B_{P}-R_{P}\right)$ colour, enabling the correction of synthetic Gaia $B_{P}$, and SkyMapper $g$ and $r$ magnitudes. We recommend that future work consider updated molecular line lists (McKemmish et al. 2019) and non-solar scaled chemical abundances (see Veyette et al. 2016).

- Using the same models, a general least squares fitting approach to medium resolution optical spectra and literature photometry is not sufficient to accurately recover $[\mathrm{Fe} / \mathrm{H}]$ for cool dwarfs. We instead develop an updated photometric $[\mathrm{Fe} / \mathrm{H}]$ calibration for cool dwarfs, built using a sample of $69 \mathrm{M}$ and $\mathrm{K}$ dwarfs with FGK binary companions having reliable $[\mathrm{Fe} / \mathrm{H}]$ measurements. By relating the position of these isolated main sequence $\mathrm{KM}$ stars in $M_{K_{S}}-\left(B_{P}-K_{S}\right)$ space to the FGK companion, and thus system, $[\mathrm{Fe} / \mathrm{H}]$, our relation can determine metallicity to a precision of \pm 0.19 dex for stars with $1.51<\left(B_{P}-R_{P}\right)<3.3$. This relation expands on the work of Bonfils et al. (2005), Johnson \& Apps (2009), and Schlaufman \& Laughlin (2010), and takes advantage of precision Gaia parallaxes (for precise distances) and kinematics (for binary identification) for the first time.

- We determine $T_{\text {eff }}$ and $R_{\star}$ for our 92 TESS candidate planet hosts with a median precision of $0.8 \%$ and $1.7 \%$ respectively, as well as radial velocities to $\sim 4.5 \mathrm{~km} \mathrm{~s}^{-1}$. 42 of these targets did not previously have radial velocities from Gaia DR2, thus completing completing the kinematics for these stars.

- We report $\mathrm{H} \alpha$ equivalent widths and $\mathrm{Ca}$ II $\mathrm{H} \& \mathrm{~K} \log R_{\mathrm{HK}}^{\prime}$ for our sample, both signs of activity and youth. None of our stars display detectable Lithium $6708 \AA$ Absorption.

- We use our derived stellar parameters to fit the TESS light curves for our 100 planet candidates in order to determine $R_{P}$ with a median precision of $3.5 \%$. Our planet properties are consistent with the 30 already confirmed by other studies. We additionally see evidence of the planet radius gap at a $\sim 1 \sigma$ level for our low-mass stellar sample, with the robustness of the detection only limited by the small sample size.

- We report the existence of two likely young systems based on stellar emission and location above the main sequence: TOI 507 (TIC 348538431) and TOI 142 (425934411). The former appears to be a near-equal mass, double-lined eclipsing binary with $T_{\text {eff }} \approx 3300 \mathrm{~K}$, potentially amenable to characterisation as a pre-main sequence benchmark system. TOI 142 on the other hand has a giant planet $\left(R_{P}=13.31 \pm 1.39 R_{\oplus}\right)$ on a short period $(T \approx 0.85$ day $)$ orbit.

This is one of the largest uniform analyses of cool TESS candidate planet hosts to date, and the first cool dwarf photometric $[\mathrm{Fe} / \mathrm{H}]$ calibration based on Gaia data. Given the major difficulties encountered using model atmospheres for $[\mathrm{Fe} / \mathrm{H}]$ determination, we plan to conduct follow-up work investigating empirical or data driven approaches built upon our now large collection of cool dwarf standard spectra.

\section{ACKNOWLEDGEMENTS}

We acknowledge the traditional custodians of the land on which the ANU $2.3 \mathrm{~m}$ Telescope stands, the Gamilaraay people, and pay our respects to elders past and present. We also acknowledge helpful early conversations with George Zhou about target selection and observing strategy, as well as the efforts of Andy Casey in developing a prototype data-driven model which ultimately proved out of scope for this study. We thank the anonymous referee for their helpful comments.

ADR acknowledges support from the Australian Government Research Training Program, and the Research School of Astronomy \& Astrophysics top up scholarship. MŽ and MJI acknowledge funding from the Australian Research Council (grant DP170102233). LC is the recipient of the ARC Future Fellowship FT160100402. MJ was supported the Research School of Astronomy and Astrophysics at the Australian National University and funding from Australian Research Council grant No. DP150100250. Parts of this research 
were conducted by the Australian Research Council Centre of Excellence for All Sky Astrophysics in 3 Dimensions (ASTRO 3D), through project number CE170100013.

This research has made use of the Exoplanet Follow-up Observation Program website, which is operated by the California Institute of Technology, under contract with the National Aeronautics and Space Administration under the Exoplanet Exploration Program. This paper includes data collected by the TESS mission. Funding for the TESS mission is provided by the NASA Explorer Program. This work has made use of data from the European Space Agency (ESA) mission Gaia (https://www . cosmos. esa.int/gaia), processed by the Gaia Data Processing and Analysis Consortium (DPAC, https://www. cosmos.esa.int/web/ gaia/dpac/consortium). Funding for the DPAC has been provided by national institutions, in particular the institutions participating in the Gaia Multilateral Agreement. This publication makes use of data products from the Two Micron All Sky Survey, which is a joint project of the University of Massachusetts and the Infrared Processing and Analysis Center/California Institute of Technology, funded by the National Aeronautics and Space Administration and the National Science Foundation. The national facility capability for SkyMapper has been funded through ARC LIEF grant LE130100104 from the Australian Research Council, awarded to the University of Sydney, the Australian National University, Swinburne University of Technology, the University of Queensland, the University of Western Australia, the University of Melbourne, Curtin University of Technology, Monash University and the Australian Astronomical Observatory. SkyMapper is owned and operated by The Australian National University's Research School of Astronomy and Astrophysics. The survey data were processed and provided by the SkyMapper Team at ANU. The SkyMapper node of the All-Sky Virtual Observatory (ASVO) is hosted at the National Computational Infrastructure (NCI). Development and support the SkyMapper node of the ASVO has been funded in part by Astronomy Australia Limited (AAL) and the Australian Government through the Commonwealth's Education Investment Fund (EIF) and National Collaborative Research Infrastructure Strategy (NCRIS), particularly the National eResearch Collaboration Tools and Resources (NeCTAR) and the Australian National Data Service Projects (ANDS). This research made use of Lightkurve, a Python package for Kepler and TESS data analysis.

Software: Astropy (Astropy Collaboration et al. 2013), batman (Kreidberg 2015), iPython (Perez \& Granger 2007), dustmaps (Green 2018), lightkurve (Lightkurve Collaboration et al. 2018), Matplotlib (Hunter 2007), NumPy (Harris et al. 2020), Pandas (McKinney 2010), SciPy (Jones et al. 2016).

\section{DATA AVAILABILITY}

All fitted stellar and planetary results are available in the article and in its online supplementary material, and stellar spectra will be shared on reasonable request to the corresponding author. All other data used is publicly available.

\section{SUPPORTING INFORMATION}

Supplementary data are available at MNRAS online.

\section{REFERENCES}

Allard F., Hauschildt P. H., Alexander D. R., Starrfield S., 1997, Annual Review of Astronomy and Astrophysics, 35, 137

Allard F., Homeier D., Freytag B., 2011, in ASP Conference Series. p. 91, http: //adsabs . harvard . edu/abs/2011ASPC . 448 . .91A

Allard F., Homeier D., Freytag B., Sharp C. M., 2012a, in EAS Publications Series. Roscoff, France, pp 3-43, doi:10.1051/eas/1257001, http: // adsabs.harvard.edu/abs/2012EAS . . . 57 . . . 3A

Allard F., Homeier D., Freytag B., 2012b, Philosophical Transactions of the Royal Society of London Series A, 370, 2765

Alvarez R., Plez B., 1998, Astronomy and Astrophysics, 330, 1109

Asplund M., Grevesse N., Sauval A. J., Scott P., 2009, Annual Review of Astronomy and Astrophysics, 47, 481

Astropy Collaboration et al., 2013, Astronomy and Astrophysics, 558, A33

Baraffe I., Chabrier G., Allard F., Hauschildt P. H., 1998, Astronomy and Astrophysics, 337, 403

Baraffe I., Homeier D., Allard F., Chabrier G., 2015, Astronomy and Astrophysics, 577, A42

Bayliss D., et al., 2018, Monthly Notices of the Royal Astronomical Society, 475,4467

Bean J. L., Sneden C., Hauschildt P. H., Johns-Krull C. M., Benedict G. F., 2006a, The Astrophysical Journal, 652, 1604

Bean J. L., Benedict G. F., Endl M., 2006b, The Astrophysical Journal Letters, 653, L65

Benedict G. F., et al., 2016, The Astronomical Journal, 152, 141

Birky J., Hogg D. W., Mann A. W., Burgasser A., 2020, The Astrophysical Journal, 892, 31

Bonfils X., Delfosse X., Udry S., Santos N. C., Forveille T., Ségransan D., 2005, Astronomy and Astrophysics, 442, 635

Borucki W. J., et al., 2010, Science, 327, 977

Boyajian T. S., et al., 2012, The Astrophysical Journal, 757, 112

Brown A. G. A., et al., 2018, Astronomy \& Astrophysics, 616, A1

Caffau E., Ludwig H.-G., Steffen M., Freytag B., Bonifacio P., 2011, Solar Physics, 268, 255

Carrillo A., Hawkins K., Bowler B. P., Cochran W., Vanderburg A., 2020, Monthly Notices of the Royal Astronomical Society, 491, 4365

Casagrande L., VandenBerg D. A., 2014, Monthly Notices of the Royal Astronomical Society, 444, 392

Casagrande L., VandenBerg D. A., 2018a, Monthly Notices of the Royal Astronomical Society, 475, 5023

Casagrande L., VandenBerg D. A., 2018b, Monthly Notices of the Royal Astronomical Society, 479, L102

Casagrande L., Schönrich R., Asplund M., Cassisi S., Ramírez I., Meléndez J., Bensby T., Feltzing S., 2011, Astronomy and Astrophysics, 530, A138

Casagrande L., Wolf C., Mackey A. D., Nordlander T., Yong D., Bessell M., 2019, Monthly Notices of the Royal Astronomical Society, 482, 2770

Casagrande L., et al., 2020, arXiv:2011.02517 [astro-ph]

Cassan A., et al., 2012, Nature, 481, 167

Chabrier G., 2003, Publications of the Astronomical Society of the Pacific, 115,763

Chabrier G., Baraffe I., 2000, Annual Review of Astronomy and Astrophysics, 38, 337

Childress M. J., Vogt F. P. A., Nielsen J., Sharp R. G., 2014, Astrophysics and Space Science, 349, 617

Claret A., 2017, Astronomy and Astrophysics, 600, A30

Cloutier R., Menou K., 2020, The Astronomical Journal, 159, 211

Crundall T. D., Ireland M. J., Krumholz M. R., Federrath C., Žerjal M., Hansen J. T., 2019, Monthly Notices of the Royal Astronomical Society, 489,3625

David T. J., et al., 2016, Nature, 534, 658

De Cat P., et al., 2015, The Astrophysical Journal Supplement Series, 220, 19

Delfosse X., Forveille T., Ségransan D., Beuzit J.-L., Udry S., Perrier C., Mayor M., 2000, Astronomy and Astrophysics, 364, 217

Desidera S., et al., 2004, Astronomy and Astrophysics, 420, 683

Dittmann J. A., Irwin J. M., Charbonneau D., Newton E. R., 2016, The

MNRAS 000, 1-21 (2015) 
Astrophysical Journal, 818, 153

Dopita M., Hart J., McGregor P., Oates P., Bloxham G., Jones D., 2007, Astrophysics and Space Science, 310, 255

Dotter A., 2016, The Astrophysical Journal Supplement Series, 222, 8

Dotter A., Chaboyer B., Jevremović D., Kostov V., Baron E., Ferguson J. W., 2008, The Astrophysical Journal Supplement Series, 178, 89

Dreizler S., et al., 2020, Astronomy \& Astrophysics, 644, A127

Dressing C. D., Charbonneau D., 2015, The Astrophysical Journal, 807, 45

Dressing C. D., et al., 2019, The Astronomical Journal, 158, 87

Esposito M., et al., 2017, Astronomy and Astrophysics, 601, A53

Feiden G. A., Chaboyer B., 2012, The Astrophysical Journal, 757, 42

Fuhrmann K., 2008, Monthly Notices of the Royal Astronomical Society, 384,173

Fulton B. J., Petigura E. A., 2018, The Astronomical Journal, 156, 264

Fulton B. J., et al., 2017, The Astronomical Journal, 154, 109

Gaia Collaboration et al., 2016, Astronomy and Astrophysics, 595, A2

Gaidos E., et al., 2014, Monthly Notices of the Royal Astronomical Society, 443,2561

Gilbert E. A., et al., 2020, The Astronomical Journal, 160, 116

Ginzburg S., Schlichting H. E., Sari R., 2018, Monthly Notices of the Royal Astronomical Society, 476, 759

Gray R. O., Corbally C. J., Garrison R. F., McFadden M. T., Bubar E. J., McGahee C. E., O’Donoghue A. A., Knox E. R., 2006, The Astronomical Journal, 132, 161

Green G. M., 2018, The Journal of Open Source Software, 3, 695

Günther M. N., et al., 2019, Nature Astronomy, 3, 1099

Gupta A., Schlichting H. E., 2019, Monthly Notices of the Royal Astronomical Society, 487, 24

Gupta A., Schlichting H. E., 2020, Monthly Notices of the Royal Astronomical Society, 493, 792

Gustafsson B., Edvardsson B., Eriksson K., Jørgensen U. G., Nordlund Å., Plez B., 2008, Astronomy and Astrophysics, 486, 951

Hansen J. T., Casagrande L., Ireland M. J., Lin J., 2021, Monthly Notices of the Royal Astronomical Society, 501, 5309

Hardegree-Ullman K. K., Zink J. K., Christiansen J. L., Dressing C. D., Ciardi D. R., Schlieder J. E., 2020, The Astrophysical Journal Supplement Series, 247,28

Harris C. R., et al., 2020, Nature, 585, 357

Hartman J. D., et al., 2015, The Astronomical Journal, 149, 166

Hartman J. D., et al., 2020, The Astronomical Journal, 159, 173

Hawkins K., et al., 2020, Monthly Notices of the Royal Astronomical Society, 492, 1164

Hejazi N., De Robertis M. M., Dawson P. C., 2015, The Astronomical Journal, 149, 140

Henry T. J., McCarthy Jr. D. W., 1993, The Astronomical Journal, 106, 773

Henry T. J., Kirkpatrick J. D., Simons D. A., 1994, The Astronomical Journal, 108, 1437

Henry T. J., Jao W.-C., Subasavage J. P., Beaulieu T. D., Ianna P. A., Costa E., Méndez R. A., 2006, The Astronomical Journal, 132, 2360

Henry T. J., et al., 2018, The Astronomical Journal, 155, 265

Hinkel N. R., et al., 2016, The Astrophysical Journal Supplement Series, 226,4

Hoeijmakers H. J., Kok R. J. d., Snellen I. a. G., Brogi M., Birkby J. L., Schwarz H., 2015, Astronomy \& Astrophysics, 575, A20

Howard A. W., et al., 2012, The Astrophysical Journal Supplement Series, 201,15

Howell S. B., et al., 2014, Publications of the Astronomical Society of the Pacific, 126, 398

Huang C. X., et al., 2020, The Astrophysical Journal, 892, L7

Hunter J. D., 2007, Computing in Science Engineering, 9, 90

Ikoma M., Hori Y., 2012, The Astrophysical Journal, 753, 66

Johnson J. A., Apps K., 2009, The Astrophysical Journal, 699, 933

Jones E., Oliphant T., Peterson P., 2016, SciPy: Open source scientific tools for Python, 2001

Joyce M., Chaboyer B., 2015, The Astrophysical Journal, 814, 142

Joyce M., Chaboyer B., 2018, The Astrophysical Journal, 856, 10

Keller S. C., et al., 2007, Publications of the Astronomical Society of Australia, 24,1
Kipping D. M., 2008, Monthly Notices of the Royal Astronomical Society, 389,1383

Kostov V. B., et al., 2019, The Astronomical Journal, 158, 32

Kraus A. L., Cody A. M., Covey K. R., Rizzuto A. C., Mann A. W., Ireland M. J., 2015, The Astrophysical Journal, 807, 3

Kreidberg L., 2015, Publications of the Astronomical Society of the Pacific, 127,1161

Kruse E., Agol E., Luger R., Foreman-Mackey D., 2019, The Astrophysical Journal Supplement Series, 244, 11

Kurucz R. L., 1995, Kurucz CD-ROM, 15. Cambridge, Mass.: Smithsonian Astrophysical Observatory

Kuruwita R. L., Ireland M., Rizzuto A., Bento J., Federrath C., 2018, Monthly Notices of the Royal Astronomical Society, 480, 5099

Kuznetsov M. K., del Burgo C., Pavlenko Y. V., Frith J., 2019, The Astrophysical Journal, 878, 134

Lallement R., Welsh B. Y., Vergely J. L., Crifo F., Sfeir D., 2003, Astronomy \& Astrophysics, 411, 447

Lebzelter T., et al., 2012, Astronomy \& Astrophysics, 547, A108

Lee E. J., Chiang E., 2016, The Astrophysical Journal, 817, 90

Lee E. J., Chiang E., Ormel C. W., 2014, The Astrophysical Journal, 797, 95

Leggett S. K., Allard F., Berriman G., Dahn C. C., Hauschildt P. H., 1996, The Astrophysical Journal Supplement Series, 104, 117

Leike R. H., Enßlin T. A., 2019, Astronomy \& Astrophysics, 631, A32

Leike R. H., Glatzle M., Enßlin T. A., 2020, Astronomy and Astrophysics, 639, A138

Leleu A., et al., 2021, Astronomy \& Astrophysics, 649, A26

Lépine S., Hilton E. J., Mann A. W., Wilde M., Rojas-Ayala B., Cruz K. L., Gaidos E., 2013, The Astronomical Journal, 145, 102

Leroy J. L., 1993, Astronomy and Astrophysics, 274, 203

Lightkurve Collaboration et al., 2018, Lightkurve: Kepler and TESS time series analysis in Python, http://adsabs.harvard.edu/abs/ 2018ascl. soft $12013 \mathrm{~L}$

Lindgren S., Heiter U., 2017, Astronomy and Astrophysics, 604, A97

Lindgren S., Heiter U., Seifahrt A., 2016, Astronomy and Astrophysics, 586, A 100

Lopez E. D., Fortney J. J., 2014, The Astrophysical Journal, 792, 1

Lopez E. D., Rice K., 2018, Monthly Notices of the Royal Astronomical Society, 479, 5303

Luck R. E., 2017, The Astronomical Journal, 153, 21

Luck R. E., 2018, The Astronomical Journal, 155, 111

Luck R. E., Heiter U., 2005, The Astronomical Journal, 129, 1063

Luque R., et al., 2019, Astronomy and Astrophysics, 628, A39

Mann A. W., Gaidos E., Lépine S., Hilton E. J., 2012, The Astrophysical Journal, 753, 90

Mann A. W., Brewer J. M., Gaidos E., Lépine S., Hilton E. J., 2013a, The Astronomical Journal, 145, 52

Mann A. W., Brewer J. M., Gaidos E., Lépine S., Hilton E. J., 2013b, Astronomische Nachrichten, 334, 18

Mann A. W., Gaidos E., Ansdell M., 2013c, The Astrophysical Journal, 779, 188

Mann A. W., Feiden G. A., Gaidos E., Boyajian T., von Braun K., 2015, The Astrophysical Journal, 804, 64

Mann A. W., et al., 2016, The Astronomical Journal, 152, 61

Mann A. W., et al., 2019, The Astrophysical Journal, 871, 63

McKemmish L. K., Masseron T., Hoeijmakers H. J., Pérez-Mesa V., Grimm S. L., Yurchenko S. N., Tennyson J., 2019, Monthly Notices of the Royal Astronomical Society, 488, 2836

McKinney W., 2010, in Proceedings of the 9th Python in Science Conference. pp 51-56, doi:10.25080/Majora-92bf192200a, http://conference.scipy.org/proceedings/scipy2010/ mckinney.html

Ment K., et al., 2019, The Astronomical Journal, 157, 32

Mishenina T. V., Soubiran C., Kovtyukh V. V., Korotin S. A., 2004, Astronomy and Astrophysics, 418, 551

Montes D., et al., 2018, Monthly Notices of the Royal Astronomical Society, 479,1332

Morton T. D., Swift J., 2014, The Astrophysical Journal, 791, 10 
Muirhead P. S., Hamren K., Schlawin E., Rojas-Ayala B., Covey K. R., Lloyd J. P., 2012, The Astrophysical Journal Letters, 750, L37

Muirhead P. S., Dressing C. D., Mann A. W., Rojas-Ayala B., Lépine S., Paegert M., De Lee N., Oelkers R., 2018, The Astronomical Journal, 155,180

Murphy S. J., et al., 2020, Monthly Notices of the Royal Astronomical Society, 491, 4902

Neves V., et al., 2012, Astronomy and Astrophysics, 538, A25

Newton E. R., Charbonneau D., Irwin J., Berta-Thompson Z. K., RojasAyala B., Covey K., Lloyd J. P., 2014, The Astronomical Journal, 147, 20

Nielsen L. D., et al., 2020, Astronomy and Astrophysics, 639, A76

Nordlander T., et al., 2019, Monthly Notices of the Royal Astronomical Society, 488, L109

Önehag A., Heiter U., Gustafsson B., Piskunov N., Plez B., Reiners A., 2012, Astronomy and Astrophysics, 542, A33

Onken C. A., et al., 2019, Publications of the Astronomical Society of Australia, 36, e033

Owen J. E., Wu Y., 2013, The Astrophysical Journal, 775, 105

Owen J. E., Wu Y., 2017, The Astrophysical Journal, 847, 29

Pagel B. E. J., Portinari L., 1998, Monthly Notices of the Royal Astronomical Society, 298,747

Passegger V. M., Wende-von Berg S., Reiners A., 2016, Astronomy and Astrophysics, 587, A19

Passegger V. M., et al., 2018, Astronomy and Astrophysics, 615, A6

Perez F., Granger B. E., 2007, Computing in Science Engineering, 9, 21

Plez B., 1998, Astronomy and Astrophysics, 337, 495

Plez B., 2012, Turbospectrum: Code for spectral synthesis, http:// adsabs. harvard. edu/abs/2012ascl. soft $05004 \mathrm{P}$

Prugniel P., Vauglin I., Koleva M., 2011, Astronomy and Astrophysics, 531, A165

Rabus M., et al., 2019, Monthly Notices of the Royal Astronomical Society, 484, 2674

Rains A. D., Ireland M. J., White T. R., Casagrande L., Karovicova I., 2020, Monthly Notices of the Royal Astronomical Society, 493, 2377

Rajpurohit A. S., Reylé C., Allard F., Homeier D., Schultheis M., Bessell M. S., Robin A. C., 2013, Astronomy and Astrophysics, 556, A15

Rajpurohit A. S., Reylé C., Allard F., Scholz R.-D., Homeier D., Schultheis M., Bayo A., 2014, Astronomy and Astrophysics, 564, A90

Ramírez I., Allende Prieto C., Lambert D. L., 2007, Astronomy and Astrophysics, 465, 271

Reylé C., Rajpurohit A. S., Schultheis M., Allard F., 2011, in ASP Conference Series. University of Washington, Seattle, Washington, p. 929, http://adsabs.harvard.edu/abs/2011ASPC . 448. .929R

Ricker G. R., et al., 2015, Journal of Astronomical Telescopes, Instruments, and Systems, 1, 014003

Robinson S. E., Ammons S. M., Kretke K. A., Strader J., Wertheimer J. G., Fischer D. A., Laughlin G., 2007, The Astrophysical Journal Supplement Series, 169, 430

Rojas-Ayala B., Covey K. R., Muirhead P. S., Lloyd J. P., 2010, The Astrophysical Journal Letters, 720, L113

Rojas-Ayala B., Covey K. R., Muirhead P. S., Lloyd J. P., 2012, The Astrophysical Journal, 748, 93

Ryabchikova T., Piskunov N., Kurucz R. L., Stempels H. C., Heiter U., Pakhomov Y., Barklem P. S., 2015, Physica Scripta, 90, 054005

Savitzky A., Golay M. J. E., 1964, Analytical Chemistry, 36, 1627

Schlaufman K. C., Laughlin G., 2010, Astronomy and Astrophysics, 519, A105

Skory S., Weck P. F., Stancil P. C., Kirby K., 2003, The Astrophysical Journal Supplement Series, 148, 599

Skrutskie M. F., et al., 2006, The Astronomical Journal, 131, 1163

Sousa S. G., Santos N. C., Israelian G., Mayor M., Monteiro M. J. P. F. G., 2006, Astronomy and Astrophysics, 458, 873

Sousa S. G., et al., 2008, Astronomy and Astrophysics, 487, 373

Sousa S. G., Santos N. C., Israelian G., Mayor M., Udry S., 2011, Astronomy and Astrophysics, 533, A141

Souto D., et al., 2017, The Astrophysical Journal, 835, 239

Souto D., et al., 2018, The Astrophysical Journal Letters, 860, L15
Sozzetti A., Torres G., Latham D. W., Stefanik R. P., Korzennik S. G., Boss A. P., Carney B. W., Laird J. B., 2009, The Astrophysical Journal, 697, 544

Stassun K. G., Torres G., 2018, The Astrophysical Journal, 862, 61

Stassun K. G., et al., 2018, The Astronomical Journal, 156, 102

Stassun K. G., et al., 2019, The Astronomical Journal, 158, 138

Terrien R. C., Mahadevan S., Bender C. F., Deshpande R., Ramsey L. W., Bochanski J. J., 2012, The Astrophysical Journal Letters, 747, L38

Tsantaki M., Sousa S. G., Adibekyan V.Z., Santos N. C., Mortier A., Israelian G., 2013, Astronomy and Astrophysics, 555, A150

Valenti J. A., Fischer D. A., 2005, The Astrophysical Journal Supplement Series, 159,141

Van Eylen V., Agentoft C., Lundkvist M. S., Kjeldsen H., Owen J. E., Fulton B. J., Petigura E., Snellen I., 2018, Monthly Notices of the Royal Astronomical Society, 479, 4786

VandenBerg D. A., Bergbusch P. A., Dowler P. D., 2006, The Astrophysical Journal Supplement Series, 162, 375

Vanderspek R., et al., 2019, The Astrophysical Journal Letters, 871, L24

Veyette M. J., Muirhead P. S., Mann A. W., Allard F., 2016, The Astrophysical Journal, 828, 95

Veyette M. J., Muirhead P. S., Mann A. W., Brewer J. M., Allard F., Homeier D., 2017, The Astrophysical Journal, 851, 26

Waalkes W. C., et al., 2020, The Astronomical Journal, 161, 13

Winters J. G., et al., 2015, The Astronomical Journal, 149, 5

Winters J. G., et al., 2019, The Astronomical Journal, 158, 152

Wittenmyer R. A., et al., 2020, Monthly Notices of the Royal Astronomical Society, 496,851

Woolf V. M., Wallerstein G., 2005, Monthly Notices of the Royal Astronomical Society, 356, 963

Woolf V. M., Wallerstein G., 2006, Publications of the Astronomical Society of the Pacific, 118, 218

Žerjal M., et al., 2021, Monthly Notices of the Royal Astronomical Society, 503, 938

Zhao G., Zhao Y.-H., Chu Y.-Q., Jing Y.-P., Deng L.-C., 2012, Research in Astronomy and Astrophysics, 12, 723

da Silva R., Milone A. C., Reddy B. E., 2011, Astronomy and Astrophysics, $526, \mathrm{~A} 71$

von Braun K., et al., 2012, The Astrophysical Journal, 753, 171

von Braun K., et al., 2014, Monthly Notices of the Royal Astronomical Society, 438,2413

\section{APPENDIX A: OBSERVATIONS \\ APPENDIX B: STELLAR STANDARDS \\ APPENDIX C: PHOTOMETRIC [FE/H] DATA \\ APPENDIX D: LIMB DARKENING \\ APPENDIX E: LITERATURE PLANETS}

This paper has been typeset from a $\mathrm{T}_{\mathrm{E}} \mathrm{X} / \mathrm{LT}_{\mathrm{E}} \mathrm{X}$ file prepared by the author. 
Table A1. Observing log for TESS candidate exoplanet host stars

\begin{tabular}{|c|c|c|c|c|c|c|}
\hline \multirow[t]{2}{*}{ TIC } & \multirow[t]{2}{*}{ UT Date } & \multirow[t]{2}{*}{ airmass } & \multirow{2}{*}{$\begin{array}{c}\exp \\
(\mathrm{sec})\end{array}$} & \multirow{2}{*}{$\begin{array}{c}\mathrm{RV} \\
\left(\mathrm{km} \mathrm{s}^{-1}\right)\end{array}$} & \multicolumn{2}{|c|}{ SNR } \\
\hline & & & & & (B) & (R) \\
\hline 219338557 & $19-08-25$ & 1.1 & 120 & $36.00 \pm 4.50$ & 66 & 185 \\
\hline 201248411 & $19-08-26$ & 1.1 & 200 & $17.92 \pm 4.50$ & 52 & 142 \\
\hline 262530407 & $19-08-26$ & 1.1 & 200 & $8.71 \pm 4.51$ & 37 & 114 \\
\hline 118327550 & $19-08-26$ & 1.0 & 300 & $6.48 \pm 4.53$ & 23 & 75 \\
\hline 410153553 & $19-08-26$ & 1.3 & 600 & $-14.96 \pm 4.55$ & 14 & 63 \\
\hline 62483237 & $19-08-26$ & 1.0 & 150 & $6.17 \pm 4.50$ & 61 & 164 \\
\hline 259377017 & $19-08-27$ & 1.3 & 300 & $20.21 \pm 4.53$ & 26 & 84 \\
\hline 200322593 & $19-08-27$ & 1.4 & 600 & $12.61 \pm 4.55$ & 12 & 46 \\
\hline 406941612 & $19-08-27$ & 1.7 & 300 & $2.38 \pm 4.52$ & 24 & 80 \\
\hline 193641523 & $19-08-27$ & 1.7 & 300 & $26.62 \pm 4.50$ & 54 & 151 \\
\hline 351601843 & $19-08-28$ & 1.4 & 300 & $26.31 \pm 4.51$ & 21 & 71 \\
\hline 234994474 & $19-08-28$ & 1.1 & 300 & $24.29 \pm 4.51$ & 71 & 208 \\
\hline 251848941 & $19-10-13$ & 1.0 & 600 & $56.79 \pm 4.50$ & 72 & 199 \\
\hline 259962054 & $19-10-14$ & 1.2 & 300 & $-11.16 \pm 4.54$ & 6 & 27 \\
\hline 101948569 & $19-10-14$ & 1.0 & 120 & $22.63 \pm 4.50$ & 28 & 82 \\
\hline 179985715 & $19-10-14$ & 1.0 & 120 & $-12.54 \pm 4.51$ & 17 & 58 \\
\hline 37749396 & $19-10-14$ & 1.1 & 60 & $-9.28 \pm 4.51$ & 52 & 149 \\
\hline 122613513 & $19-10-14$ & 1.0 & 300 & $8.72 \pm 4.50$ & 54 & 145 \\
\hline 229111835 & $19-10-14$ & 1.1 & 300 & $13.15 \pm 4.51$ & 12 & 42 \\
\hline 70899085 & $19-10-15$ & 1.1 & 300 & $-12.74 \pm 4.51$ & 30 & 96 \\
\hline 98796344 & $19-10-15$ & 1.1 & 90 & $-10.03 \pm 4.54$ & 30 & 96 \\
\hline 55488511 & $19-10-15$ & 1.1 & 300 & $89.66 \pm 4.51$ & 18 & 63 \\
\hline 271596225 & $19-10-15$ & 1.4 & 300 & $-0.68 \pm 4.52$ & 15 & 53 \\
\hline 44647437 & $19-10-16$ & 1.0 & 300 & $14.43 \pm 4.51$ & 9 & 36 \\
\hline 237914496 & $19-10-16$ & 1.2 & 300 & $-6.74 \pm 4.51$ & 18 & 58 \\
\hline 237920046 & $19-10-16$ & 1.2 & 300 & $8.94 \pm 4.51$ & 12 & 43 \\
\hline 201642601 & $19-10-17$ & 1.3 & 900 & $-27.52 \pm 4.51$ & 16 & 57 \\
\hline 158297421 & $19-10-17$ & 1.2 & 900 & $-5.31 \pm 4.50$ & 14 & 49 \\
\hline 261108236 & $19-10-17$ & 1.5 & 600 & $4.02 \pm 4.51$ & 19 & 69 \\
\hline 231702397 & $19-10-17$ & 1.1 & 900 & $-76.33 \pm 4.54$ & 7 & 33 \\
\hline 322063810 & $19-10-17$ & 1.1 & 150 & $32.28 \pm 4.51$ & 47 & 137 \\
\hline 220479565 & $19-10-17$ & 1.1 & 600 & $30.73 \pm 4.53$ & 10 & 47 \\
\hline 220459976 & $19-10-17$ & 1.2 & 600 & $15.25 \pm 4.51$ & 20 & 73 \\
\hline 192826603 & $19-10-17$ & 1.1 & 1200 & $95.80 \pm 4.52$ & 7 & 38 \\
\hline 44737596 & $19-10-17$ & 1.1 & 1200 & $41.46 \pm 4.52$ & 6 & 36 \\
\hline 38510224 & $19-10-17$ & 1.2 & 300 & $29.91 \pm 4.50$ & 23 & 72 \\
\hline 33831980 & $19-10-17$ & 1.4 & 600 & $11.84 \pm 4.51$ & 25 & 85 \\
\hline 219229644 & $19-10-17$ & 1.1 & 300 & $48.73 \pm 4.51$ & 28 & 91 \\
\hline 14165625 & $19-10-17$ & 1.1 & 300 & $22.75 \pm 4.50$ & 27 & 86 \\
\hline 32497972 & $19-10-17$ & 1.0 & 300 & $3.57 \pm 4.51$ & 16 & 60 \\
\hline 170849515 & $19-10-18$ & 1.0 & 1800 & $4.86 \pm 4.52$ & 9 & 29 \\
\hline 100608026 & $19-10-19$ & 1.0 & 300 & $24.98 \pm 4.51$ & 29 & 97 \\
\hline 144700903 & $19-10-19$ & 1.4 & 700 & $-0.04 \pm 4.51$ & 12 & 53 \\
\hline 12423815 & $19-11-16$ & 1.0 & 2000 & $-11.69 \pm 4.52$ & 9 & 35 \\
\hline 260708537 & $20-02-01$ & 1.1 & 120 & $25.15 \pm 4.53$ & 32 & 102 \\
\hline 35009898 & $20-02-01$ & 1.2 & 600 & $-41.91 \pm 4.53$ & 14 & 52 \\
\hline 151825527 & $20-02-01$ & 1.0 & 450 & $-5.31 \pm 4.52$ & 23 & 80 \\
\hline 150428135 & $20-02-01$ & 1.2 & 360 & $-4.25 \pm 4.53$ & 29 & 92 \\
\hline 260004324 & $20-02-01$ & 1.1 & 180 & $41.76 \pm 4.52$ & 32 & 99 \\
\hline 167600516 & $20-02-01$ & 1.2 & 300 & $36.55 \pm 4.51$ & 45 & 136 \\
\hline 219195044 & $20-02-01$ & 1.1 & 450 & $117.69 \pm 4.52$ & 28 & 90 \\
\hline 73649615 & $20-02-01$ & 1.0 & 700 & $14.53 \pm 4.52$ & 12 & 50 \\
\hline 165317334 & $20-02-01$ & 1.0 & 150 & $4.98 \pm 4.51$ & 22 & 80 \\
\hline 440887364 & $20-02-01$ & 1.1 & 45 & $-25.52 \pm 4.50$ & 46 & 140 \\
\hline 307210830 & $20-02-03$ & 1.3 & 120 & $-5.17 \pm 4.53$ & 23 & 88 \\
\hline 141608198 & $20-02-03$ & 1.4 & 900 & $14.91 \pm 4.54$ & 8 & 44 \\
\hline 33521996 & $20-02-03$ & 1.0 & 900 & $41.68 \pm 4.52$ & 6 & 37 \\
\hline 19025965 & $20-02-03$ & 1.6 & 360 & $46.78 \pm 4.50$ & 17 & 73 \\
\hline 27649847 & $20-02-03$ & 1.4 & 720 & $-10.40 \pm 4.53$ & 8 & 44 \\
\hline 413248763 & $20-02-03$ & 1.0 & 45 & $-36.03 \pm 4.53$ & 22 & 80 \\
\hline 296739893 & $20-02-03$ & 1.1 & 300 & $8.47 \pm 4.52$ & 38 & 118 \\
\hline 36734222 & $20-02-03$ & 1.1 & 450 & $-8.65 \pm 4.51$ & 24 & 89 \\
\hline 54962195 & $20-02-03$ & 1.1 & 450 & $25.13 \pm 4.52$ & 7 & 44 \\
\hline 158588995 & $20-02-03$ & 1.0 & 720 & $7.25 \pm 4.53$ & 9 & 49 \\
\hline
\end{tabular}


Table A1 - continued Observing log for TESS candidate exoplanet host stars

\begin{tabular}{|c|c|c|c|c|c|c|}
\hline \multirow[t]{2}{*}{ TIC } & \multirow{2}{*}{ UT Date } & \multirow{2}{*}{ airmass } & \multirow{2}{*}{$\begin{array}{l}\exp \\
(\mathrm{sec})\end{array}$} & \multirow{2}{*}{$\begin{array}{c}\mathrm{RV} \\
\left(\mathrm{km} \mathrm{s}^{-1}\right)\end{array}$} & \multicolumn{2}{|c|}{ SNR } \\
\hline & & & & & (B) & (R) \\
\hline 141527579 & $20-02-03$ & 1.4 & 900 & $24.59 \pm 4.52$ & 20 & 75 \\
\hline 149788158 & $20-02-03$ & 1.2 & 360 & $24.03 \pm 4.52$ & 19 & 73 \\
\hline 34068865 & $20-02-03$ & 1.1 & 30 & $50.46 \pm 4.52$ & 30 & 99 \\
\hline 36724087 & $20-02-03$ & 1.1 & 360 & $-7.62 \pm 4.53$ & 14 & 60 \\
\hline 359271092 & $20-02-03$ & 1.2 & 20 & $37.39 \pm 4.51$ & 24 & 91 \\
\hline 429358906 & $20-02-03$ & 1.1 & 1200 & $-1.21 \pm 4.54$ & 6 & 43 \\
\hline 374829238 & $20-02-03$ & 1.2 & 600 & $15.29 \pm 4.51$ & 19 & 73 \\
\hline 300710077 & $20-02-03$ & 1.3 & 660 & $58.98 \pm 4.53$ & 12 & 60 \\
\hline 362249359 & $20-02-03$ & 1.2 & 180 & $14.78 \pm 4.51$ & 32 & 110 \\
\hline 210873792 & $20-02-03$ & 1.4 & 450 & $-75.36 \pm 4.51$ & 22 & 78 \\
\hline 261257684 & $20-02-03$ & 1.6 & 300 & $7.56 \pm 4.51$ & 25 & 91 \\
\hline 280437559 & $20-02-03$ & 1.3 & 180 & $-3.64 \pm 4.50$ & 25 & 91 \\
\hline 370133522 & 20-09-11 & 1.3 & 180 & $-0.93 \pm 4.53$ & 20 & 70 \\
\hline 12421862 & 20-09-11 & 1.0 & 180 & $19.29 \pm 4.51$ & 28 & 97 \\
\hline 415969908 & 20-09-11 & 1.0 & 450 & $24.75 \pm 4.52$ & 20 & 71 \\
\hline 92226327 & 20-09-11 & 1.1 & 450 & $-15.10 \pm 4.55$ & 9 & 44 \\
\hline 348538431 & $20-09-11$ & 2.0 & 900 & $-6.72 \pm 4.56$ & 15 & 56 \\
\hline 254113311 & $20-09-12$ & 1.0 & 180 & $-7.33 \pm 4.50$ & 39 & 115 \\
\hline 29960110 & $20-09-12$ & 1.0 & 360 & $26.52 \pm 4.52$ & 17 & 63 \\
\hline 425934411 & $20-09-12$ & 1.2 & 1200 & $-47.79 \pm 4.53$ & 11 & 52 \\
\hline 153065527 & $20-09-12$ & 1.0 & 450 & $17.50 \pm 4.54$ & 8 & 41 \\
\hline 50618703 & $20-09-12$ & 1.3 & 120 & $10.43 \pm 4.50$ & 36 & 111 \\
\hline 77156829 & $20-09-12$ & 1.0 & 300 & $9.06 \pm 4.53$ & 21 & 77 \\
\hline 231728511 & $20-09-12$ & 1.1 & 450 & $-5.94 \pm 4.53$ & 13 & 51 \\
\hline 369327947 & $20-09-12$ & 1.6 & 300 & $-13.78 \pm 4.53$ & 23 & 79 \\
\hline 175532955 & $20-09-12$ & 1.0 & 360 & $76.23 \pm 4.50$ & 25 & 79 \\
\hline 141527965 & $20-09-13$ & 1.4 & 450 & $37.43 \pm 4.50$ & 29 & 90 \\
\hline 260417932 & $20-09-13$ & 1.2 & 300 & $36.85 \pm 4.50$ & 42 & 116 \\
\hline
\end{tabular}


Table B1. Observing log for cool dwarf standards

\begin{tabular}{|c|c|c|c|c|c|c|}
\hline \multirow[t]{2}{*}{ Gaia DR2 } & \multirow[t]{2}{*}{ UT Date } & \multirow[t]{2}{*}{ airmass } & \multirow{2}{*}{$\begin{array}{c}\exp \\
(\mathrm{sec})\end{array}$} & \multirow{2}{*}{$\begin{array}{c}\mathrm{RV} \\
\left(\mathrm{km} \mathrm{s}^{-1}\right)\end{array}$} & \multicolumn{2}{|c|}{ SNR } \\
\hline & & & & & (B) & (R) \\
\hline 19316224572460416 & $19-07-22$ & 1.5 & 5 & $20.65 \pm 4.50$ & 108 & 251 \\
\hline 2306965202564506752 & $19-07-22$ & 1.0 & 200 & $19.60 \pm 4.52$ & 180 & 487 \\
\hline 2603090003484152064 & $19-07-22$ & 1.1 & 300 & $-4.68 \pm 4.53$ & 100 & 281 \\
\hline 2683023811628007296 & $19-07-22$ & 1.3 & 300 & $19.01 \pm 4.51$ & 159 & 462 \\
\hline 2828928008202069376 & $19-07-22$ & 1.5 & 200 & $-30.56 \pm 4.51$ & 158 & 458 \\
\hline 5134635708766250752 & $19-07-22$ & 1.1 & 300 & $8.56 \pm 4.51$ & 182 & 529 \\
\hline 6553614253923452800 & $19-07-22$ & 1.0 & 30 & $7.13 \pm 4.51$ & 117 & 335 \\
\hline 6604147121141267712 & $19-07-22$ & 1.0 & 30 & $8.13 \pm 4.50$ & 192 & 474 \\
\hline 2552925644460225152 & $19-08-26$ & 1.2 & 10 & $-14.82 \pm 4.50$ & 144 & 309 \\
\hline 2627117287488522240 & $19-08-26$ & 1.5 & 60 & $-18.10 \pm 4.53$ & 92 & 247 \\
\hline 2653762573103102592 & $19-08-26$ & 1.5 & 90 & $11.04 \pm 4.51$ & 94 & 271 \\
\hline 2702655587447223168 & $19-08-26$ & 1.6 & 300 & $-13.88 \pm 4.51$ & 74 & 219 \\
\hline 2739689239311660672 & $19-08-26$ & 1.2 & 90 & $-79.67 \pm 4.52$ & 73 & 213 \\
\hline 4282578724832056576 & $19-08-26$ & 1.7 & 120 & $9.19 \pm 4.51$ & 82 & 234 \\
\hline 4293318823182081408 & $19-08-26$ & 1.7 & 100 & $30.79 \pm 4.52$ & 85 & 228 \\
\hline 6412595290592307840 & $19-08-26$ & 1.1 & 6 & $-42.60 \pm 4.50$ & 296 & 711 \\
\hline 6562924609150908416 & $19-08-26$ & 1.1 & 60 & $7.74 \pm 4.52$ & 180 & 492 \\
\hline 2611163717366876544 & $19-08-27$ & 1.2 & 60 & $-11.00 \pm 4.51$ & 45 & 139 \\
\hline 2815543034682035840 & $19-08-27$ & 1.6 & 90 & $-7.36 \pm 4.51$ & 44 & 136 \\
\hline 3209938366665770752 & $19-08-27$ & 2.6 & 30 & $2.25 \pm 4.51$ & 66 & 209 \\
\hline 114207651462714880 & $19-10-12$ & 1.9 & 300 & $26.03 \pm 4.53$ & 114 & 346 \\
\hline 2515037264041041536 & $19-10-12$ & 1.2 & 120 & $-10.79 \pm 4.52$ & 110 & 309 \\
\hline 2560771312759450496 & $19-10-12$ & 1.2 & 180 & $-31.93 \pm 4.52$ & 77 & 227 \\
\hline 301785537751949824 & $19-10-12$ & 2.0 & 300 & $-9.94 \pm 4.51$ & 65 & 211 \\
\hline 87921523897823872 & $19-10-12$ & 1.7 & 300 & $-4.49 \pm 4.52$ & 65 & 197 \\
\hline 145421309108301184 & $19-10-14$ & 1.7 & 60 & $-35.17 \pm 4.51$ & 93 & 282 \\
\hline 3210731015767419520 & $19-10-14$ & 1.2 & 30 & $-55.79 \pm 4.50$ & 109 & 278 \\
\hline 3339921875389105152 & $19-10-14$ & 1.4 & 120 & $20.00 \pm 4.51$ & 108 & 318 \\
\hline 3359074685047632640 & $19-10-14$ & 1.8 & 200 & $-60.17 \pm 4.51$ & 91 & 282 \\
\hline 3409711211681795584 & $19-10-14$ & 1.6 & 180 & $23.09 \pm 4.52$ & 78 & 233 \\
\hline 1736838805468812160 & $19-10-15$ & 1.4 & 30 & $-70.90 \pm 4.50$ & 58 & 162 \\
\hline 3057712188691831936 & $19-10-15$ & 1.2 & 90 & $-20.08 \pm 4.51$ & 92 & 274 \\
\hline 4075141768785646848 & $19-10-15$ & 1.5 & 60 & $-9.52 \pm 4.55$ & 32 & 110 \\
\hline 4971496564348094336 & $19-10-15$ & 1.0 & 90 & $43.73 \pm 4.52$ & 52 & 148 \\
\hline 5006921282807193856 & $19-10-15$ & 1.0 & 150 & $40.49 \pm 4.51$ & 61 & 179 \\
\hline 2910909931633597312 & $19-10-17$ & 1.0 & 144 & $53.69 \pm 4.52$ & 44 & 125 \\
\hline 2979590513145784192 & $19-10-17$ & 1.0 & 144 & $-15.39 \pm 4.52$ & 43 & 131 \\
\hline 3101920046552857728 & $19-10-17$ & 1.2 & 90 & $-11.34 \pm 4.52$ & 65 & 184 \\
\hline 3117120863523946368 & $19-10-17$ & 1.2 & 90 & $11.51 \pm 4.55$ & 25 & 91 \\
\hline 3136952686035250688 & $19-10-17$ & 1.4 & 90 & $19.87 \pm 4.56$ & 25 & 87 \\
\hline 3184351876391975808 & $19-10-17$ & 1.1 & 90 & $-10.63 \pm 4.52$ & 44 & 130 \\
\hline 3316364602541746048 & $19-10-17$ & 1.2 & 144 & $24.40 \pm 4.54$ & 31 & 104 \\
\hline 5951824121022278144 & $19-10-17$ & 1.5 & 108 & $-8.80 \pm 4.53$ & 75 & 223 \\
\hline 3057712223051571200 & $20-02-01$ & 1.1 & 12 & $-13.82 \pm 4.50$ & 99 & 231 \\
\hline 4364527594192166400 & $20-02-01$ & 2.1 & 15 & $36.77 \pm 4.51$ & 65 & 195 \\
\hline 5378886891122066560 & $20-02-01$ & 1.0 & 15 & $14.90 \pm 4.50$ & 81 & 206 \\
\hline 6143175840404555008 & $20-02-01$ & 1.0 & 24 & $-4.07 \pm 4.50$ & 35 & 101 \\
\hline 6223456372670070656 & $20-02-01$ & 1.1 & 90 & $18.75 \pm 4.50$ & 79 & 202 \\
\hline 6288325222245417856 & $20-02-01$ & 1.0 & 15 & $-36.71 \pm 4.50$ & 63 & 184 \\
\hline 1244644727396803584 & $20-02-03$ & 1.6 & 60 & $24.60 \pm 4.51$ & 46 & 152 \\
\hline 3089711447388931584 & $20-02-03$ & 1.2 & 60 & $65.99 \pm 4.52$ & 44 & 138 \\
\hline 3195919322830293760 & $20-02-03$ & 1.1 & 144 & $-45.15 \pm 4.55$ & 41 & 120 \\
\hline 3256334497479041024 & $20-02-03$ & 1.2 & 15 & $13.50 \pm 4.50$ & 55 & 152 \\
\hline 3520548825260557312 & $20-02-03$ & 1.0 & 60 & $112.47 \pm 4.51$ & 64 & 158 \\
\hline 3550084490721711872 & $20-02-03$ & 1.1 & 90 & $30.33 \pm 4.50$ & 51 & 143 \\
\hline 3630092241022731136 & $20-02-03$ & 1.1 & 36 & $-15.90 \pm 4.50$ & 51 & 144 \\
\hline 3661492609484469504 & $20-02-03$ & 1.2 & 60 & $-37.17 \pm 4.50$ & 75 & 210 \\
\hline 3689602277083844480 & $20-02-03$ & 1.2 & 72 & $8.91 \pm 4.51$ & 105 & 321 \\
\hline 3738099879558957952 & $20-02-03$ & 1.4 & 72 & $18.94 \pm 4.51$ & 79 & 240 \\
\hline 3741297293732404352 & $20-02-03$ & 1.5 & 45 & $19.02 \pm 4.52$ & 80 & 235 \\
\hline 3767878708888402816 & $20-02-03$ & 1.2 & 90 & $26.64 \pm 4.51$ & 53 & 163 \\
\hline 3796072592206250624 & $20-02-03$ & 1.2 & 120 & $-34.21 \pm 4.54$ & 21 & 90 \\
\hline 3828238392559860992 & $20-02-03$ & 1.3 & 120 & $10.18 \pm 4.52$ & 92 & 268 \\
\hline 3855208897392952192 & $20-02-03$ & 1.3 & 120 & $6.61 \pm 4.52$ & 70 & 211 \\
\hline
\end{tabular}


Table B1 - continued Observing log for cool dwarf standards

\begin{tabular}{|c|c|c|c|c|c|c|}
\hline \multirow[t]{2}{*}{ Gaia DR2 } & \multirow[t]{2}{*}{ UT Date } & \multirow[t]{2}{*}{ airmass } & \multirow{2}{*}{$\begin{array}{c}\exp \\
(\mathrm{sec})\end{array}$} & \multirow{2}{*}{$\begin{array}{c}\mathrm{RV} \\
\left(\mathrm{km} \mathrm{s}^{-1}\right)\end{array}$} & \multicolumn{2}{|c|}{ SNR } \\
\hline & & & & & (B) & (R) \\
\hline 3902785109124370432 & $20-02-03$ & 1.4 & 300 & $20.95 \pm 4.51$ & 106 & 322 \\
\hline 4017860992519744384 & $20-02-03$ & 1.9 & 120 & $4.28 \pm 4.53$ & 33 & 118 \\
\hline 4330690742322011520 & $20-02-03$ & 1.4 & 120 & $-25.48 \pm 4.53$ & 62 & 195 \\
\hline 4364480521350598144 & $20-02-03$ & 1.8 & 60 & $31.88 \pm 4.52$ & 39 & 130 \\
\hline 5170039502144332800 & $20-02-03$ & 1.2 & 15 & $-1.55 \pm 4.50$ & 58 & 164 \\
\hline 5266270443442455040 & $20-02-03$ & 1.3 & 90 & $18.99 \pm 4.50$ & 70 & 183 \\
\hline 614543647497149056 & $20-02-03$ & 1.6 & 90 & $12.23 \pm 4.52$ & 36 & 118 \\
\hline 6232511606838403968 & $20-02-03$ & 1.2 & 7 & $27.29 \pm 4.50$ & 130 & 330 \\
\hline 6324325225803432320 & $20-02-03$ & 1.2 & 144 & $-1.32 \pm 4.54$ & 38 & 122 \\
\hline 116037376250217984 & $20-09-11$ & 1.9 & 500 & $25.26 \pm 4.53$ & 28 & 99 \\
\hline 1750765357185849856 & $20-09-11$ & 1.5 & 500 & $-25.65 \pm 4.54$ & 25 & 96 \\
\hline 18565464288396416 & $20-09-11$ & 1.3 & 240 & $20.98 \pm 4.53$ & 36 & 112 \\
\hline 2029432043779954432 & $20-09-11$ & 2.4 & 600 & $-48.01 \pm 4.54$ & 11 & 52 \\
\hline 2316867885320845696 & $20-09-11$ & 1.0 & 150 & $16.65 \pm 4.52$ & 35 & 109 \\
\hline 2358524597030794112 & $20-09-11$ & 1.1 & 240 & $18.71 \pm 4.56$ & 26 & 98 \\
\hline 2435125446129139712 & $20-09-11$ & 1.1 & 450 & $-30.75 \pm 4.53$ & 27 & 94 \\
\hline 2533723464155234176 & $20-09-11$ & 1.3 & 150 & $13.99 \pm 4.51$ & 46 & 144 \\
\hline 2595284016771502080 & $20-09-11$ & 1.0 & 600 & $-14.03 \pm 4.55$ & 23 & 86 \\
\hline 2640434056928150400 & $20-09-11$ & 1.2 & 500 & $-41.88 \pm 4.56$ & 9 & 64 \\
\hline 2817999068780218368 & $20-09-11$ & 1.6 & 240 & $-7.29 \pm 4.54$ & 32 & 103 \\
\hline 4145870293808914688 & $20-09-11$ & 1.1 & 920 & $-46.19 \pm 4.55$ & 21 & 66 \\
\hline 4177731838628465408 & $20-09-11$ & 1.2 & 36 & $23.09 \pm 4.52$ & 43 & 132 \\
\hline 4187836934609063552 & $20-09-11$ & 1.1 & 1500 & $-58.36 \pm 4.54$ & 31 & 102 \\
\hline 4303294035005560704 & $20-09-11$ & 1.5 & 450 & $-0.34 \pm 4.53$ & 37 & 117 \\
\hline 4339465360508118912 & $20-09-11$ & 1.2 & 240 & $12.31 \pm 4.54$ & 34 & 111 \\
\hline 4365609170043180416 & $20-09-11$ & 1.2 & 360 & $-11.77 \pm 4.55$ & 31 & 105 \\
\hline 4375233191015944192 & $20-09-11$ & 1.3 & 15 & $-22.90 \pm 4.51$ & 65 & 197 \\
\hline 4389844948935164544 & $20-09-11$ & 1.3 & 45 & $-14.11 \pm 4.51$ & 47 & 148 \\
\hline 4472832130942575872 & $20-09-11$ & 1.3 & 30 & $-109.09 \pm 4.56$ & 29 & 106 \\
\hline 4498055584805914752 & $20-09-11$ & 1.7 & 920 & $-28.75 \pm 4.54$ & 32 & 107 \\
\hline 4550763526539955712 & $20-09-11$ & 1.7 & 45 & $-11.93 \pm 4.51$ & 38 & 123 \\
\hline 4572835535272064768 & $20-09-11$ & 2.2 & 60 & $2.41 \pm 4.51$ & 41 & 136 \\
\hline 4584638930036248192 & $20-09-11$ & 2.5 & 600 & $-2.63 \pm 4.52$ & 31 & 108 \\
\hline 5042734468172061440 & $20-09-11$ & 1.1 & 240 & $-41.49 \pm 4.52$ & 49 & 149 \\
\hline 5853498713160606720 & $20-09-11$ & 1.4 & 60 & $-26.88 \pm 4.56$ & 49 & 187 \\
\hline 6245870673116518784 & $20-09-11$ & 1.2 & 180 & $-2.02 \pm 4.53$ & 38 & 113 \\
\hline 6322070093095493504 & $20-09-11$ & 1.5 & 120 & $-9.91 \pm 4.53$ & 42 & 133 \\
\hline 6882963066420161664 & $20-09-11$ & 1.2 & 150 & $6.16 \pm 4.54$ & 27 & 96 \\
\hline 6885776098199761024 & $20-09-11$ & 1.2 & 150 & $-66.40 \pm 4.52$ & 41 & 131 \\
\hline 6890353330746858368 & $20-09-11$ & 1.2 & 240 & $27.46 \pm 4.53$ & 42 & 124 \\
\hline 76868614540049408 & $20-0$ & 1.5 & 240 & $-34.31 \pm 4.56$ & 21 & 83 \\
\hline 102162639019033600 & $20-09-12$ & 1.9 & 600 & $-10.88 \pm 4.54$ & 21 & 79 \\
\hline 1784473016438653056 & $20-09-12$ & 1.6 & 60 & $-27.82 \pm 4.53$ & 32 & 106 \\
\hline 1809359465717142656 & $20-09-12$ & 1.6 & 500 & $-51.83 \pm 4.54$ & 20 & 64 \\
\hline 1810616448010879488 & $20-09-12$ & 1.6 & 450 & $-62.47 \pm 4.54$ & 27 & 98 \\
\hline 1814070838668707840 & $20-09-12$ & 1.8 & 90 & $5.78 \pm 4.51$ & 40 & 129 \\
\hline 1838855376946302720 & $20-09-12$ & 1.9 & 294 & $-31.81 \pm 4.53$ & 30 & 104 \\
\hline 2507016253701863040 & $20-09-12$ & 1.2 & 450 & $21.18 \pm 4.54$ & 26 & 89 \\
\hline 2976850598890038784 & $20-09-12$ & 1.2 & 240 & $9.31 \pm 4.53$ & 28 & 95 \\
\hline 3187115498866675456 & $20-09-12$ & 1.4 & 240 & $32.96 \pm 4.55$ & 23 & 85 \\
\hline 3219851121121855872 & $20-09-12$ & 1.3 & 500 & $31.72 \pm 4.52$ & 38 & 120 \\
\hline 3239548631415741440 & $20-09-12$ & 1.6 & 360 & $40.94 \pm 4.52$ & 35 & 111 \\
\hline 3288082758293022848 & $20-09-12$ & 1.7 & 240 & $-15.01 \pm 4.53$ & 27 & 88 \\
\hline 35398295820372864 & $20-09-12$ & 1.6 & 600 & $-37.31 \pm 4.54$ & 20 & 74 \\
\hline 4295619138933719808 & $20-09-12$ & 1.4 & 600 & $19.28 \pm 4.54$ & 30 & 96 \\
\hline 4393265392167891712 & $20-09-12$ & 1.4 & 600 & $11.05 \pm 4.54$ & 22 & 76 \\
\hline 4508377078422114944 & $20-09-12$ & 1.5 & 360 & $-48.72 \pm 4.54$ & 26 & 82 \\
\hline 4512525016089353472 & $20-09-12$ & 1.7 & 500 & $11.51 \pm 4.55$ & 31 & 108 \\
\hline 4519789081415296128 & $20-09-12$ & 1.9 & 150 & $30.19 \pm 4.53$ & 40 & 134 \\
\hline 4519789321933481856 & $20-09-12$ & 1.9 & 150 & $32.15 \pm 4.53$ & 40 & 135 \\
\hline 4535928365908540416 & $20-09-12$ & 2.2 & 240 & $22.56 \pm 4.54$ & 17 & 56 \\
\hline 5077642283022422656 & $20-09-12$ & 1.0 & 90 & $31.72 \pm 4.51$ & 42 & 130 \\
\hline 2467732906559754496 & $20-09-13$ & 1.1 & 500 & $-5.83 \pm 4.56$ & 21 & 80 \\
\hline 2549050931124387456 & $20-09-13$ & 1.2 & 600 & $-5.17 \pm 4.53$ & 31 & 100 \\
\hline
\end{tabular}


Table B1 - continued Observing log for cool dwarf standards

\begin{tabular}{ccccccc}
\hline Gaia DR2 & UT Date & airmass & exp & \multicolumn{2}{c}{ RV } & \multicolumn{2}{c}{ SNR } \\
& & & $(\mathrm{sec})$ & $\left(\mathrm{km} \mathrm{s}^{-1}\right)$ & $(\mathrm{B})$ & $(\mathrm{R})$ \\
\hline 2848646203058386560 & $20-09-13$ & 1.7 & 450 & $-48.92 \pm 4.53$ & 37 & 116 \\
2868199402451064064 & $20-09-13$ & 2.2 & 600 & $-15.06 \pm 4.56$ & 21 & 85 \\
2911981886751531648 & $20-09-13$ & 1.1 & 240 & $4.93 \pm 4.53$ & 34 & 106 \\
3011648664439643520 & $20-09-13$ & 1.1 & 120 & $21.03 \pm 4.50$ & 46 & 127 \\
3131777319158582272 & $20-09-13$ & 1.4 & 300 & $-6.02 \pm 4.52$ & 28 & 97 \\
3266980243936341248 & $20-09-13$ & 1.2 & 600 & $82.84 \pm 4.52$ & 28 & 93 \\
4706630501049679744 & $20-09-13$ & 1.4 & 60 & $39.09 \pm 4.50$ & 96 & 243 \\
4871271406553197184 & $20-09-13$ & 1.0 & 90 & $26.97 \pm 4.50$ & 53 & 140 \\
5142772953804963456 & $20-09-13$ & 1.1 & 600 & $-2.48 \pm 4.53$ & 38 & 119 \\
6391176735363140480 & $20-09-13$ & 1.4 & 30 & $4.49 \pm 4.50$ & 69 & 192 \\
\hline
\end{tabular}


Table B2. Final results for cool dwarf standards

\begin{tabular}{|c|c|c|c|c|c|c|c|}
\hline Gaia DR2 & $\begin{array}{l}T_{\text {eff }} \\
(\mathrm{K})\end{array}$ & $\log g$ & {$[\mathrm{Fe} / \mathrm{H}]$} & $\begin{array}{c}M \\
\left(M_{\odot}\right)\end{array}$ & $\begin{array}{l}R_{\star} \\
\left(R_{\odot}\right)\end{array}$ & $m_{\text {bol }}$ & $\begin{array}{c}f_{\mathrm{bol}} \\
\left(10^{-12} \mathrm{ergs} \mathrm{s}^{-1} \mathrm{~cm}^{-2}\right)\end{array}$ \\
\hline 5853498713160606720 & $2885 \pm 30$ & $5.14 \pm 0.02$ & - & $0.124 \pm 0.003$ & $0.156 \pm 0.003$ & $7.36 \pm 0.01$ & $28793.2 \pm 291.5$ \\
\hline 2640434056928150400 & $2927 \pm 30$ & $5.20 \pm 0.02$ & - & $0.120 \pm 0.003$ & $0.144 \pm 0.003$ & $11.19 \pm 0.01$ & $851.2 \pm 8.9$ \\
\hline 2595284016771502080 & $3006 \pm 30$ & $5.03 \pm 0.02$ & - & $0.176 \pm 0.004$ & $0.212 \pm 0.004$ & $10.25 \pm 0.01$ & $2020.5 \pm 21.1$ \\
\hline 2467732906559754496 & $3022 \pm 30$ & $5.10 \pm 0.02$ & - & $0.145 \pm 0.004$ & $0.178 \pm 0.004$ & $11.43 \pm 0.01$ & $680.0 \pm 7.0$ \\
\hline 4393265392167891712 & $3035 \pm 30$ & $5.03 \pm 0.02$ & - & $0.181 \pm 0.004$ & $0.214 \pm 0.004$ & $11.71 \pm 0.01$ & $524.2 \pm 5.4$ \\
\hline 4145870293808914688 & $3061 \pm 30$ & $5.08 \pm 0.02$ & - & $0.153 \pm 0.004$ & $0.187 \pm 0.004$ & $10.65 \pm 0.01$ & $1398.5 \pm 14.2$ \\
\hline 2358524597030794112 & $3062 \pm 30$ & $5.13 \pm 0.02$ & -0.29 & $0.137 \pm 0.003$ & $0.167 \pm 0.003$ & $9.24 \pm 0.01$ & $5113.3 \pm 52.0$ \\
\hline 1810616448010879488 & $3064 \pm 30$ & $4.99 \pm 0.02$ & - & $0.190 \pm 0.005$ & $0.231 \pm 0.005$ & $10.58 \pm 0.01$ & $1488.6 \pm 14.8$ \\
\hline 76868614540049408 & $3069 \pm 30$ & $5.09 \pm 0.02$ & -0.17 & $0.148 \pm 0.003$ & $0.182 \pm 0.004$ & $9.45 \pm 0.01$ & $4221.2 \pm 42.1$ \\
\hline 2868199402451064064 & $3088 \pm 30$ & $5.06 \pm 0.02$ & -0.15 & $0.156 \pm 0.004$ & $0.192 \pm 0.004$ & $11.58 \pm 0.01$ & $591.2 \pm 5.9$ \\
\hline 3117120863523946368 & $3094 \pm 30$ & $4.91 \pm 0.02$ & - & $0.236 \pm 0.006$ & $0.281 \pm 0.006$ & $8.29 \pm 0.01$ & $12313.6 \pm 123.2$ \\
\hline 1750765357185849856 & $3121 \pm 30$ & $5.04 \pm 0.02$ & - & $0.183 \pm 0.004$ & $0.215 \pm 0.004$ & $11.30 \pm 0.01$ & $765.6 \pm 7.7$ \\
\hline 2029432043779954432 & $3128 \pm 30$ & $4.98 \pm 0.02$ & 0.23 & $0.206 \pm 0.005$ & $0.242 \pm 0.005$ & $11.51 \pm 0.01$ & $632.4 \pm 6.3$ \\
\hline 3796072592206250624 & $3136 \pm 30$ & $5.06 \pm 0.02$ & -0.08 & $0.176 \pm 0.004$ & $0.206 \pm 0.004$ & $8.47 \pm 0.01$ & $10415.1 \pm 105.8$ \\
\hline 2549050931124387456 & $3139 \pm 30$ & $4.72 \pm 0.02$ & - & $0.384 \pm 0.009$ & $0.445 \pm 0.009$ & $12.05 \pm 0.01$ & $385.1 \pm 3.9$ \\
\hline 3187115498866675456 & $3142 \pm 30$ & $5.08 \pm 0.02$ & -0.14 & $0.171 \pm 0.004$ & $0.198 \pm 0.004$ & $9.56 \pm 0.01$ & $3810.3 \pm 38.7$ \\
\hline 3195919322830293760 & $3160 \pm 30$ & $4.94 \pm 0.02$ & - & $0.231 \pm 0.006$ & $0.269 \pm 0.005$ & $8.72 \pm 0.01$ & $8276.6 \pm 84.2$ \\
\hline 4512525016089353472 & $3167 \pm 30$ & $4.98 \pm 0.02$ & - & $0.207 \pm 0.005$ & $0.244 \pm 0.005$ & $11.16 \pm 0.01$ & $869.9 \pm 8.7$ \\
\hline 102162639019033600 & $3169 \pm 30$ & $4.98 \pm 0.02$ & 0.05 & $0.212 \pm 0.005$ & $0.246 \pm 0.005$ & $10.39 \pm 0.01$ & $1773.4 \pm 17.7$ \\
\hline 6882963066420161664 & $3173 \pm 30$ & $4.97 \pm 0.02$ & - & $0.232 \pm 0.006$ & $0.261 \pm 0.005$ & $9.01 \pm 0.01$ & $6339.3 \pm 64.0$ \\
\hline 4472832130942575872 & $3188 \pm 30$ & $5.06 \pm 0.02$ & -0.51 & $0.160 \pm 0.004$ & $0.195 \pm 0.004$ & 7.19 & $33821.5 \pm 338.4$ \\
\hline 4535928365908540416 & $3201 \pm 30$ & $4.83 \pm 0.02$ & 0.65 & $0.342 \pm 0.008$ & $0.370 \pm 0.007$ & $9.45=$ & $4204.3 \pm 42.0$ \\
\hline 4075141768785646848 & $3204 \pm 30$ & $5.06 \pm 0.02$ & -0.32 & $0.177 \pm 0.004$ & $0.207 \pm 0.004$ & $8.10 \pm$ & $14680.7 \pm 146.2$ \\
\hline 3316364602541746048 & $3206 \pm 30$ & $4.97 \pm 0.02$ & 0.05 & $0.231 \pm 0.006$ & $0.262 \pm 0.005$ & $8.79 \pm 0.01$ & $7711.6 \pm 76.7$ \\
\hline 4187836934609063552 & $3208 \pm 30$ & $4.98 \pm 0.02$ & -0.01 & $0.230 \pm 0.006$ & $0.258 \pm 0.005$ & $11.48 \pm 0.01$ & $649.8 \pm 6.5$ \\
\hline 1809359465717142656 & $3221 \pm 30$ & $4.92 \pm 0.02$ & - & $0.244 \pm 0.006$ & $0.282 \pm 0.005$ & $11.56 \pm 0.01$ & $605.2 \pm 6.0$ \\
\hline 4365609170043180416 & $3224 \pm 30$ & $4.98 \pm 0.02$ & -0.02 & $0.235 \pm 0.006$ & $0.260 \pm 0.005$ & $9.90 \pm 0.01$ & $2796.5 \pm 28.1$ \\
\hline 2507016253701863040 & $3227 \pm 30$ & $4.97 \pm 0.02$ & 0.21 & $0.256 \pm 0.006$ & $0.275 \pm 0.005$ & $10.99 \pm 0.01$ & $1017.2 \pm 10.3$ \\
\hline 18565464288396416 & $3234 \pm 30$ & $4.92 \pm 0.02$ & 0.02 & $0.252 \pm 0.006$ & $0.288 \pm 0.005$ & $9.26 \pm 0.01$ & $5022.8 \pm 50.2$ \\
\hline 3136952686035250688 & $3241 \pm 30$ & $4.88 \pm 0.02$ & 0.44 & $0.315 \pm 0.007$ & $0.336 \pm 0.006$ & $8.51 \pm 0.01$ & $10006.1 \pm 101.8$ \\
\hline 4339465360508118912 & $3247 \pm 30$ & $5.01 \pm 0.02$ & - & $0.209 \pm 0.005$ & $0.237 \pm 0.004$ & $9.44 \pm 0.01$ & $4269.1 \pm 42.5$ \\
\hline 2848646203058386560 & $3250 \pm 30$ & $4.78 \pm 0.02$ & - & $0.373 \pm 0.009$ & $0.410 \pm 0.008$ & $10.73 \pm 0.01$ & $1293.0 \pm 12.9$ \\
\hline 3288082758293022848 & $3250 \pm 30$ & $4.81 \pm 0.02$ & - & $0.366 \pm 0.009$ & $0.393 \pm 0.007$ & $9.73 \pm 0.01$ & $3258.4 \pm 32.9$ \\
\hline 4508377078422114944 & $3265 \pm 30$ & $4.89 \pm 0.02$ & 0.14 & $0.294 \pm 0.007$ & $0.322 \pm 0.006$ & $10.09 \pm 0.01$ & $2345.5 \pm 23.3$ \\
\hline 4303294035005560704 & $3277 \pm 30$ & $4.82 \pm 0.02$ & - & $0.354 \pm 0.009$ & $0.383 \pm 0.007$ & $10.75 \pm 0.01$ & $1279.4 \pm 12.7$ \\
\hline 2435125446129139712 & $3278 \pm 30$ & $4.95 \pm 0.02$ & 0.32 & $0.303 \pm 0.008$ & $0.305 \pm 0.006$ & 10.93 & $1081.2 \pm 11.1$ \\
\hline 116037376250217984 & $3282 \pm 30$ & $4.90 \pm($ & 0.19 & $0.304 \pm 0.007$ & $0.324 \pm 0.006$ & 11.4 & $692.3 \pm 6.9$ \\
\hline 6324325225803432320 & $3283 \pm 30$ & $4.96 \pm 0.02$ & 0.32 & $0.294 \pm 0.008$ & $0.299 \pm 0.006$ & $8.80 \pm 0.01$ & $7681.5 \pm 78.9$ \\
\hline 4295619138933719808 & $3299 \pm 30$ & $5.01 \pm 0.02$ & -0.37 & $0.220 \pm 0.005$ & $0.242 \pm 0.004$ & $10.39 \pm 0.01$ & $1781.8 \pm 17.9$ \\
\hline 1784473016438653056 & $3303 \pm 30$ & $4.76 \pm 0.02$ & - & $0.395 \pm 0.009$ & $0.433 \pm 0.008$ & $8.14 \pm 0.01$ & $14033.5 \pm 139.9$ \\
\hline 4330690742322011520 & $3309 \pm 30$ & $4.91 \pm 0.02$ & 0.07 & $0.302 \pm 0.008$ & $0.321 \pm 0.006$ & $7.80 \pm 0.01$ & $19231.6 \pm 191.5$ \\
\hline 2603090003484152064 & $3326 \pm 30$ & $4.89 \pm 0.02$ & 0.29 & $0.338 \pm 0.008$ & $0.347 \pm 0.006$ & $7.79 \pm 0.01$ & $19393.6 \pm 194.0$ \\
\hline 35398295820372864 & $3330 \pm 30$ & $4.81 \pm 0.02$ & - & $0.370 \pm 0.010$ & $0.396 \pm 0.009$ & $10.57 \pm 0.01$ & $1508.8 \pm 15.0$ \\
\hline 2976850598890038784 & $3337 \pm 30$ & $4.96 \pm 0.02$ & - & $0.274 \pm 0.007$ & $0.287 \pm 0.005$ & $9.66 \pm 0.01$ & $3465.4 \pm 34.6$ \\
\hline 2817999068780218368 & $3344 \pm 30$ & $4.83 \pm 0.02$ & 0.39 & $0.381 \pm 0.009$ & $0.394 \pm 0.007$ & $9.26 \pm 0.01$ & $5021.5 \pm 50.0$ \\
\hline 6322070093095493504 & $3360 \pm 30$ & $4.90 \pm 0.02$ & -0.10 & $0.311 \pm 0.008$ & $0.327 \pm 0.006$ & $8.52 \pm 0.01$ & $9900.6 \pm 98.6$ \\
\hline 6245870673116518784 & $3360 \pm 30$ & $4.69 \pm 0.02$ & - & $0.481 \pm 0.011$ & $0.515 \pm 0.009$ & $9.35 \pm 0.01$ & $4619.8 \pm 46.8$ \\
\hline 2911981886751531648 & $3362 \pm 30$ & $4.92 \pm 0.02$ & -0.13 & $0.305 \pm 0.007$ & $0.316 \pm 0.006$ & $9.88 \pm 0.01$ & $2831.9 \pm 28.5$ \\
\hline 4498055584805914752 & $3385 \pm 30$ & $4.97 \pm 0.02$ & -0.36 & $0.259 \pm 0.006$ & $0.276 \pm 0.005$ & $11.28 \pm 0.01$ & $785.0 \pm 7.8$ \\
\hline 114207651462714880 & $3403 \pm 30$ & $4.84 \pm 0.02$ & -0.08 & $0.357 \pm 0.009$ & $0.374 \pm 0.007$ & $8.60 \pm 0.01$ & $9191.7 \pm 91.6$ \\
\hline 5951824121022278144 & $3435 \pm 30$ & $4.88 \pm 0.02$ & -0.13 & $0.353 \pm 0.008$ & $0.359 \pm 0.006$ & $7.52 \pm 0.01$ & $25039.0 \pm 249.3$ \\
\hline 3219851121121855872 & $3456 \pm 30$ & $4.69 \pm 0.02$ & - & $0.498 \pm 0.012$ & $0.524 \pm 0.009$ & $11.50 \pm 0.01$ & $636.8 \pm 6.4$ \\
\hline 4017860992519744384 & $3459 \pm 30$ & $4.79 \pm 0.02$ & 0.05 & $0.423 \pm 0.010$ & $0.434 \pm 0.008$ & $8.73 \pm 0.01$ & $8170.7 \pm 81.3$ \\
\hline 5142772953804963456 & $3483 \pm 30$ & $4.89 \pm 0.02$ & - & $0.352 \pm 0.008$ & $0.354 \pm 0.006$ & $12.06 \pm 0.01$ & $379.6 \pm 3.8$ \\
\hline 1838855376946302720 & $3484 \pm 30$ & $4.93 \pm 0.02$ & - & $0.311 \pm 0.008$ & $0.315 \pm 0.006$ & $10.24 \pm 0.01$ & $2033.8 \pm 20.3$ \\
\hline 2560771312759450496 & $3497 \pm 30$ & $4.84 \pm 0.02$ & -0.13 & $0.403 \pm 0.010$ & $0.402 \pm 0.007$ & $9.17 \pm 0.01$ & $5445.7 \pm 54.4$ \\
\hline 2627117287488522240 & $3505 \pm 30$ & $4.77 \pm 0.02$ & 0.29 & $0.465 \pm 0.011$ & $0.463 \pm 0.008$ & $8.31 \pm 0.01$ & $12075.4 \pm 120.5$ \\
\hline 6890353330746858368 & $3511 \pm 30$ & $4.76 \pm 0.02$ & 0.17 & $0.468 \pm 0.011$ & $0.470 \pm 0.008$ & $9.58 \pm 0.01$ & $3738.4 \pm 37.5$ \\
\hline 4584638930036248192 & $3516 \pm 30$ & $4.76 \pm 0.02$ & -0.05 & $0.448 \pm 0.011$ & $0.460 \pm 0.008$ & $10.85 \pm 0.01$ & $1166.3 \pm 11.6$ \\
\hline 2979590513145784192 & $3522 \pm 30$ & $4.83 \pm 0.02$ & -0.19 & $0.409 \pm 0.010$ & $0.407 \pm 0.007$ & $9.23 \pm 0.01$ & $5167.2 \pm 51.5$ \\
\hline 3239548631415741440 & $3530 \pm 30$ & $4.48 \pm 0.02$ & - & $0.624 \pm 0.015$ & $0.747 \pm 0.013$ & $10.28 \pm 0.01$ & $1955.6 \pm 19.5$ \\
\hline 3101920046552857728 & $3533 \pm 30$ & $4.79 \pm 0.02$ & -0.07 & $0.441 \pm 0.012$ & $0.445 \pm 0.008$ & $8.35 \pm 0.01$ & $11628.5 \pm 115.7$ \\
\hline 3089711447388931584 & $3534 \pm 30$ & $4.79 \pm 0.02$ & -0.09 & $0.442 \pm 0.011$ & $0.444 \pm 0.008$ & $8.40 \pm 0.01$ & $11122.0 \pm 110.7$ \\
\hline 3855208897392952192 & $3540 \pm 30$ & $4.81 \pm 0.02$ & -0.11 & $0.431 \pm 0.010$ & $0.429 \pm 0.007$ & $7.95 \pm 0.01$ & $16866.3 \pm 167.9$ \\
\hline
\end{tabular}


Table B2 - continued Final results for cool dwarf standards

\begin{tabular}{|c|c|c|c|c|c|c|c|}
\hline Gaia DR2 & $\begin{array}{l}T_{\text {eff }} \\
(\mathrm{K})\end{array}$ & $\log g$ & {$[\mathrm{Fe} / \mathrm{H}]$} & $\begin{array}{c}M \\
\left(M_{\odot}\right)\end{array}$ & $\begin{array}{c}R_{\star} \\
\left(R_{\odot}\right)\end{array}$ & $m_{\text {bol }}$ & $\begin{array}{c}f_{\mathrm{bol}} \\
\left(10^{-12} \operatorname{ergs~s}^{-1} \mathrm{~cm}^{-2}\right)\end{array}$ \\
\hline 4519789081415296128 & $3541 \pm 30$ & $4.94 \pm 0.02$ & - & $0.319 \pm 0.008$ & $0.319 \pm 0.005$ & $9.08 \pm 0.01$ & $5906.7 \pm 58.9$ \\
\hline 4519789321933481856 & $3543 \pm 30$ & $4.94 \pm 0.02$ & - & $0.318 \pm 0.008$ & $0.317 \pm 0.005$ & $9.09 \pm 0.01$ & $5856.8 \pm 58.4$ \\
\hline 3131777319158582272 & $3545 \pm 30$ & $4.65 \pm 0.02$ & - & $0.528 \pm 0.013$ & $0.566 \pm 0.010$ & $10.46 \pm 0.01$ & $1659.9 \pm 16.5$ \\
\hline 3184351876391975808 & $3555 \pm 30$ & $4.75 \pm 0.02$ & - & $0.470 \pm 0.011$ & $0.478 \pm 0.008$ & $8.70 \pm 0.01$ & $8389.8 \pm 83.5$ \\
\hline 4293318823182081408 & $3555 \pm 30$ & $4.76 \pm 0.02$ & 0.07 & $0.474 \pm 0.011$ & $0.476 \pm 0.008$ & $7.32 \pm 0.01$ & $29932.3 \pm 298.2$ \\
\hline 3409711211681795584 & $3560 \pm 30$ & $4.73 \pm 0.02$ & 0.06 & $0.489 \pm 0.012$ & $0.500 \pm 0.009$ & $8.24 \pm 0.01$ & $12905.3 \pm 128.4$ \\
\hline 2910909931633597312 & $3582 \pm 30$ & $4.71 \pm 0.02$ & 0.26 & $0.517 \pm 0.012$ & $0.527 \pm 0.009$ & $8.95 \pm 0.01$ & $6698.1 \pm 66.7$ \\
\hline 614543647497149056 & $3583 \pm 30$ & $4.75 \pm 0.02$ & -0.07 & $0.474 \pm 0.011$ & $0.478 \pm 0.008$ & $8.73 \pm 0.01$ & $8157.1 \pm 81.2$ \\
\hline 87921523897823872 & $3601 \pm 30$ & $4.72 \pm 0.02$ & 0.10 & $0.511 \pm 0.012$ & $0.518 \pm 0.009$ & $8.97 \pm 0.01$ & $6591.2 \pm 65.6$ \\
\hline 6885776098199761024 & $3611 \pm 30$ & $4.91 \pm 0.02$ & -0.56 & $0.355 \pm 0.009$ & $0.345 \pm 0.006$ & $9.46 \pm 0.01$ & $4186.0 \pm 41.8$ \\
\hline 4364480521350598144 & $3615 \pm 30$ & $4.77 \pm 0.02$ & -0.21 & $0.464 \pm 0.011$ & $0.467 \pm 0.008$ & $8.53 \pm 0.01$ & $9818.2 \pm 97.8$ \\
\hline 6562924609150908416 & $3615 \pm 30$ & $4.81 \pm 0.02$ & -0.22 & $0.440 \pm 0.010$ & $0.434 \pm 0.007$ & $7.07 \pm 0.01$ & $37591.6 \pm 374.6$ \\
\hline 4971496564348094336 & $3618 \pm 30$ & $4.71 \pm 0.02$ & 0.04 & $0.516 \pm 0.012$ & $0.526 \pm 0.009$ & $8.71 \pm 0.01$ & $8361.5 \pm 83.2$ \\
\hline 2316867885320845696 & $3622 \pm 30$ & $4.71 \pm 0.02$ & 0.16 & $0.525 \pm 0.012$ & $0.530 \pm 0.009$ & $9.43 \pm 0.01$ & $4283.9 \pm 42.6$ \\
\hline 3828238392559860992 & $3627 \pm 30$ & $4.72 \pm 0.02$ & 0.03 & $0.512 \pm 0.012$ & $0.518 \pm 0.009$ & $7.63 \pm 0.01$ & $22534.9 \pm 224.3$ \\
\hline 2515037264041041536 & $3635 \pm 30$ & $4.79 \pm 0.02$ & -0.33 & $0.449 \pm 0.011$ & $0.449 \pm 0.008$ & $8.60 \pm 0.01$ & $9214.0 \pm 92.0$ \\
\hline 4550763526539955712 & $3638 \pm 30$ & $4.81 \pm 0.02$ & -0.32 & $0.442 \pm 0.010$ & $0.436 \pm 0.007$ & $8.12 \pm 0.01$ & $14421.3 \pm 143.6$ \\
\hline 3767878708888402816 & $3647 \pm 30$ & $4.70 \pm 0.02$ & -0.01 & $0.526 \pm 0.013$ & $0.538 \pm 0.009$ & $8.63 \pm 0.01$ & $8991.2 \pm 89.5$ \\
\hline 4177731838628465408 & $3649 \pm 30$ & $4.79 \pm 0.02$ & -0.22 & $0.466 \pm 0.011$ & $0.456 \pm 0.008$ & $7.89 \pm 0.01$ & $17811.0 \pm 177.3$ \\
\hline 6553614253923452800 & $3656 \pm 30$ & $4.74 \pm 0.04$ & -0.35 & $0.463 \pm 0.034$ & $0.479 \pm 0.008$ & $5.91 \pm 0.01$ & $109647.8 \pm 1093.2$ \\
\hline 3741297293732404352 & $3657 \pm 30$ & $4.75 \pm 0.02$ & -0.19 & $0.487 \pm 0.011$ & $0.486 \pm 0.008$ & $6.97 \pm 0.01$ & $41290.1 \pm 411.5$ \\
\hline 5042734468172061440 & $3660 \pm 30$ & $4.77 \pm 0.02$ & - & $0.484 \pm 0.011$ & $0.476 \pm 0.008$ & $9.68 \pm 0.01$ & $3399.1 \pm 33.9$ \\
\hline 2739689239311660672 & $3673 \pm 30$ & $4.85 \pm 0.02$ & -0.44 & $0.413 \pm 0.010$ & $0.399 \pm 0.007$ & $7.56 \pm 0.01$ & $23962.9 \pm 238.6$ \\
\hline 4572835535272064768 & $3680 \pm 30$ & $4.72 \pm 0.02$ & -0.09 & $0.518 \pm 0.012$ & $0.523 \pm 0.009$ & $8.19 \pm 0.01$ & $13424.8 \pm 133.7$ \\
\hline 2306965202564506752 & $3683 \pm 30$ & $4.90 \pm 0.02$ & - & $0.389 \pm 0.009$ & $0.368 \pm 0.006$ & & $38086.6 \pm 379.9$ \\
\hline 2828928008202069376 & $3687 \pm 30$ & $4.67 \pm 0.02$ & 0.09 & $0.551 \pm 0.013$ & $0.564 \pm 0.009$ & $7.12 \pm 0.01$ & $36004.7 \pm 358.9$ \\
\hline 3209938366665770752 & $3693 \pm 30$ & $4.58 \pm 0.04$ & -0.02 & $0.562 \pm 0.042$ & $0.635 \pm 0.010$ & $6.45 \pm 0.01$ & $66571.3 \pm 665.3$ \\
\hline 3738099879558957952 & $3707 \pm 30$ & $4.75 \pm 0.02$ & -0.16 & $0.505 \pm 0.012$ & $0.495 \pm 0.008$ & $7.61 \pm 0.01$ & $23016.6 \pm 229.1$ \\
\hline 4389844948935164544 & $3734 \pm 30$ & $4.69 \pm 0.02$ & -0.13 & $0.541 \pm 0.014$ & $0.551 \pm 0.009$ & $7.96 \pm 0.01$ & $16663.8 \pm 166.0$ \\
\hline 3359074685047632640 & $3746 \pm 30$ & $4.79 \pm 0.02$ & -0.44 & $0.467 \pm 0.011$ & $0.454 \pm 0.007$ & $8.34 \pm 0.01$ & $11698.3 \pm 116.7$ \\
\hline 3266980243936341248 & $3756 \pm 30$ & $4.92 \pm 0.02$ & - & $0.376 \pm 0.009$ & $0.352 \pm 0.006$ & $13.32 \pm 0.01$ & $119.5 \pm 1.2$ \\
\hline 2683023811628007296 & $3802 \pm 30$ & $4.67 \pm 0.02$ & -0.05 & $0.571 \pm 0.014$ & $0.578 \pm 0.009$ & $7.87 \pm 0.01$ & $18086.3 \pm 180.1$ \\
\hline 4282578724832056576 & $3810 \pm 30$ & $4.66 \pm 0.02$ & 0.02 & $0.582 \pm 0.014$ & $0.593 \pm 0.009$ & $7.92 \pm 0.01$ & $17330.6 \pm 172.6$ \\
\hline 3902785109124370432 & $3813 \pm 30$ & $4.68 \pm 0.02$ & - & $0.567 \pm 0.014$ & $0.571 \pm 0.010$ & $8.43 \pm 0.01$ & $10831.2 \pm 107.8$ \\
\hline 2611163717366876544 & $3828 \pm 30$ & $4.70 \pm 0.02$ & -0.15 & $0.557 \pm 0.014$ & $0.554 \pm 0.009$ & $8.62 \pm 0.01$ & $9063.8 \pm 90.2$ \\
\hline 2653762573103102592 & $3831 \pm 30$ & $4.65 \pm 0.02$ & 0.06 & $0.591 \pm 0.015$ & $0.604 \pm 0.010$ & $8.72 \pm 0.01$ & $8284.3 \pm 82.4$ \\
\hline 1814070838668707840 & $3859 \pm 30$ & $4.61 \pm 0.03$ & - & $0.604 \pm 0.016$ & $0.634 \pm 0.017$ & $9.06 \pm 0.01$ & $6068.2 \pm 60.6$ \\
\hline 5006921282807193856 & $3860 \pm 30$ & $4.74 \pm 0.02$ & -0.31 & $0.538 \pm 0.013$ & $0.520 \pm 0.008$ & $9.38 \pm 0.01$ & $4514.1 \pm 44.9$ \\
\hline 2702655587447223168 & $3866 \pm 30$ & $4.64 \pm 0.02$ & 0.02 & $0.599 \pm 0.015$ & $0.611 \pm 0.010$ & $9.29 \pm 0.01$ & $4900.3 \pm 48.8$ \\
\hline 1244644727396803584 & $3888 \pm 30$ & $4.80 \pm 0.02$ & -0.50 & $0.499 \pm 0.012$ & $0.470 \pm 0.007$ & $8.66 \pm 0.01$ & $8747.3 \pm 87.1$ \\
\hline 301785537751949824 & $3948 \pm 30$ & $4.64 \pm 0.02$ & 0.12 & $0.619 \pm 0.015$ & $0.624 \pm 0.010$ & $9.13 \pm 0.01$ & $5640.6 \pm 56.2$ \\
\hline 5134635708766250752 & $3958 \pm 30$ & $4.66 \pm 0.02$ & 0.03 & $0.608 \pm 0.015$ & $0.605 \pm 0.009$ & $7.70 \pm 0.01$ & $21175.6 \pm 211.0$ \\
\hline 3339921875389105152 & $3974 \pm 30$ & $4.66 \pm 0.02$ & -0.06 & $0.605 \pm 0.015$ & $0.605 \pm 0.009$ & $7.75 \pm 0.01$ & $20128.4 \pm 200.4$ \\
\hline 2533723464155234176 & $4030 \pm 30$ & $4.68 \pm 0.02$ & - & $0.602 \pm 0.015$ & $0.591 \pm 0.011$ & $9.64 \pm 0.01$ & $3540.0 \pm 35.3$ \\
\hline 5077642283022422656 & $4033 \pm 30$ & $4.64 \pm 0.02$ & -0.07 & $0.617 \pm 0.015$ & $0.622 \pm 0.009$ & $9.18 \pm 0.01$ & $5410.4 \pm 54.0$ \\
\hline 3689602277083844480 & $4044 \pm 30$ & $4.62 \pm 0.02$ & 0.10 & $0.638 \pm 0.016$ & $0.648 \pm 0.010$ & $7.37 \pm 0.01$ & $28564.9 \pm 284.8$ \\
\hline 4375233191015944192 & $4064 \pm 30$ & $4.55 \pm 0.03$ & -0.36 & $0.611 \pm 0.043$ & $0.682 \pm 0.010$ & $6.54 \pm 0.01$ & $61522.8 \pm 614.6$ \\
\hline 2815543034682035840 & $4132 \pm 30$ & $4.66 \pm 0.02$ & -0.12 & $0.625 \pm 0.015$ & $0.613 \pm 0.009$ & $9.27 \pm 0.01$ & $4998.8 \pm 49.8$ \\
\hline 3057712188691831936 & $4154 \pm 30$ & $4.66 \pm 0.02$ & -0.13 & $0.627 \pm 0.015$ & $0.616 \pm 0.009$ & $7.97 \pm 0.01$ & $16501.3 \pm 164.5$ \\
\hline 145421309108301184 & $4157 \pm 30$ & $4.61 \pm 0.02$ & 0.08 & $0.656 \pm 0.016$ & $0.668 \pm 0.010$ & $7.30 \pm 0.01$ & $30485.1 \pm 304.2$ \\
\hline 3630092241022731136 & $4325 \pm 30$ & $4.61 \pm 0.02$ & - & $0.665 \pm 0.016$ & $0.669 \pm 0.009$ & $8.43 \pm 0.01$ & $10747.0 \pm 107.2$ \\
\hline 6288325222245417856 & $4403 \pm 30$ & $4.58 \pm 0.02$ & -0.04 & $0.685 \pm 0.017$ & $0.699 \pm 0.010$ & $7.44 \pm 0.01$ & $26824.7 \pm 267.3$ \\
\hline 6391176735363140480 & $4408 \pm 30$ & $4.60 \pm 0.02$ & - & $0.678 \pm 0.017$ & $0.686 \pm 0.010$ & $7.59 \pm 0.01$ & $23309.6 \pm 232.2$ \\
\hline 4364527594192166400 & $4409 \pm 30$ & $4.66 \pm 0.02$ & - & $0.655 \pm 0.016$ & $0.628 \pm 0.009$ & $7.03 \pm 0.01$ & $39249.2 \pm 391.7$ \\
\hline 1736838805468812160 & $4419 \pm 30$ & $4.58 \pm 0.02$ & - & $0.685 \pm 0.017$ & $0.699 \pm 0.010$ & $7.57 \pm 0.01$ & $23790.6 \pm 237.7$ \\
\hline 3011648664439643520 & $4433 \pm 30$ & $4.72 \pm 0.02$ & - & $0.621 \pm 0.015$ & $0.568 \pm 0.008$ & $10.01 \pm 0.01$ & $2513.1 \pm 25.0$ \\
\hline 3661492609484469504 & $4469 \pm 30$ & $4.58 \pm 0.02$ & - & $0.691 \pm 0.017$ & $0.708 \pm 0.010$ & $8.56 \pm 0.01$ & $9562.0 \pm 95.2$ \\
\hline 3550084490721711872 & $4502 \pm 30$ & $4.61 \pm 0.02$ & - & $0.674 \pm 0.017$ & $0.671 \pm 0.009$ & $9.32 \pm 0.01$ & $4744.6 \pm 47.3$ \\
\hline 4871271406553197184 & $4517 \pm 30$ & $4.63 \pm 0.02$ & - & $0.670 \pm 0.016$ & $0.660 \pm 0.009$ & $9.55 \pm 0.01$ & $3842.3 \pm 38.3$ \\
\hline 4706630501049679744 & $4529 \pm 30$ & $4.67 \pm 0.02$ & - & $0.653 \pm 0.016$ & $0.617 \pm 0.008$ & $7.92 \pm 0.01$ & $17249.1 \pm 171.8$ \\
\hline 6232511606838403968 & $4548 \pm 30$ & $4.53 \pm 0.03$ & - & $0.721 \pm 0.045$ & $0.763 \pm 0.010$ & $5.22 \pm 0.01$ & $207838.3 \pm 2069.6$ \\
\hline 3210731015767419520 & $4554 \pm 30$ & $4.58 \pm 0.02$ & - & $0.700 \pm 0.018$ & $0.712 \pm 0.010$ & $7.07 \pm 0.01$ & $37634.1 \pm 375.1$ \\
\hline 5170039502144332800 & $4571 \pm 30$ & $4.54 \pm 0.02$ & - & $0.720 \pm 0.021$ & $0.756 \pm 0.010$ & $7.30 \pm 0.01$ & $30580.6 \pm 305.1$ \\
\hline 3256334497479041024 & $4576 \pm 30$ & $4.61 \pm 0.02$ & - & $0.686 \pm 0.017$ & $0.681 \pm 0.009$ & $7.51 \pm 0.01$ & $25118.9 \pm 250.2$ \\
\hline
\end{tabular}


Table B2 - continued Final results for cool dwarf standards

\begin{tabular}{|c|c|c|c|c|c|c|c|}
\hline Gaia DR2 & $\begin{array}{l}T_{\text {eff }} \\
(\mathrm{K})\end{array}$ & $\log g$ & {$[\mathrm{Fe} / \mathrm{H}]$} & $\begin{array}{c}M \\
\left(M_{\odot}\right)\end{array}$ & $\begin{array}{c}R_{\star} \\
\left(R_{\odot}\right)\end{array}$ & $m_{\text {bol }}$ & $\begin{array}{c}f_{\mathrm{bol}} \\
\left(10^{-12} \operatorname{ergs~s}^{-1} \mathrm{~cm}^{-2}\right)\end{array}$ \\
\hline 5378886891122066560 & $4611 \pm 30$ & $4.66 \pm 0.02$ & - & $0.664 \pm 0.016$ & $0.634 \pm 0.008$ & $7.25 \pm 0.01$ & $32106.9 \pm 319.6$ \\
\hline 6143175840404555008 & $4612 \pm 30$ & $4.57 \pm 0.02$ & - & $0.706 \pm 0.019$ & $0.724 \pm 0.010$ & $7.90 \pm 0.01$ & $17587.4 \pm 175.1$ \\
\hline 6604147121141267712 & $4636 \pm 30$ & $4.61 \pm 0.03$ & - & $0.690 \pm 0.042$ & $0.677 \pm 0.009$ & $5.95 \pm 0.01$ & $105643.6 \pm 1053.2$ \\
\hline 3520548825260557312 & $4667 \pm 30$ & $4.70 \pm 0.02$ & - & $0.642 \pm 0.016$ & $0.589 \pm 0.008$ & $9.00 \pm 0.01$ & $6412.7 \pm 63.8$ \\
\hline 6412595290592307840 & $4670 \pm 30$ & $4.57 \pm 0.03$ & - & $0.685 \pm 0.041$ & $0.711 \pm 0.009$ & $4.22 \pm 0.01$ & $523617.3 \pm 5227.6$ \\
\hline 19316224572460416 & $4808 \pm 30$ & $4.53 \pm 0.03$ & - & $0.724 \pm 0.043$ & $0.765 \pm 0.010$ & $5.42 \pm 0.01$ & $172178.2 \pm 1715.9$ \\
\hline 3057712223051571200 & $4917 \pm 30$ & $4.56 \pm 0.02$ & - & $0.729 \pm 0.022$ & $0.739 \pm 0.009$ & $6.85 \pm 0.01$ & $46403.6 \pm 463.8$ \\
\hline 2552925644460225152 & $5011 \pm 30$ & $4.57 \pm 0.03$ & - & $0.702 \pm 0.048$ & $0.717 \pm 0.009$ & $5.44 \pm 0.01$ & $169339.3 \pm 1688.1$ \\
\hline
\end{tabular}


Table C1. Stellar pairs and primary $[\mathrm{Fe} / \mathrm{H}]$ used for photometric $[\mathrm{Fe} / \mathrm{H}]$ relation

\begin{tabular}{|c|c|c|c|c|}
\hline Gaia DR2 ID (s) & $B_{P}-R_{P}$ & Gaia DR2 ID (p) & {$[\mathrm{Fe} / \mathrm{H}] \mathrm{ref}$} & {$[\mathrm{Fe} / \mathrm{H}]$ adopted } \\
\hline 853820948481913472 & 1.66 & 853819947756949120 & Valenti \& Fischer 2005 & $-0.07 \pm 0.03$ \\
\hline 3057712188691831936 & 1.71 & 3057712223051571200 & Valenti \& Fischer 2005 & $0.07 \pm 0.03$ \\
\hline 3732696295305334016 & 1.73 & 3732696398384550400 & Mann et al. 2013c & $-0.57 \pm 0.03$ \\
\hline 3999917031474342016 & 1.94 & 3999916962754865152 & Ramírez et al. 2007 & $-0.72 \pm 0.05$ \\
\hline 3936909723803146496 & 2.03 & 3936909723803146368 & Mann et al. 2013c & $-0.12 \pm 0.03$ \\
\hline 3266980243936341248 & 2.04 & 3266980170921153920 & Mann et al. 2013c & $-0.92 \pm 0.03$ \\
\hline 4364480521350598144 & 2.18 & 4364527594192166400 & Valenti \& Fischer 2005 & $-0.41 \pm 0.03$ \\
\hline 2719475542667351552 & 2.26 & 2719475542666622976 & Valenti \& Fischer 2005 & $-0.22 \pm 0.03$ \\
\hline 3131777319158582272 & 2.26 & 3131777319158581376 & Valenti \& Fischer 2005 & $0.25 \pm 0.03$ \\
\hline 3239548631415741440 & 2.29 & 3239548665775613056 & Valenti \& Fischer 2005 & $0.05 \pm 0.03$ \\
\hline 4584638930036248192 & 2.32 & 4584639307993378432 & Valenti \& Fischer 2005 & $-0.06 \pm 0.03$ \\
\hline 3101920046552857728 & 2.33 & 3101923001490347392 & Valenti \& Fischer 2005 & $0.14 \pm 0.03$ \\
\hline 3219851121121855872 & 2.40 & 3219847066672970368 & Valenti \& Fischer 2005 & $0.45 \pm 0.03$ \\
\hline 466291787445425408 & 2.43 & 466294295706341760 & da Silva et al. 2011 & $-0.25 \pm 0.03$ \\
\hline 1982947131682334848 & 2.49 & 1982946891164168576 & Valenti \& Fischer 2005 & $0.00 \pm 0.03$ \\
\hline 4303294035005560704 & 2.68 & 4303294039306246656 & Valenti \& Fischer 2005 & $0.16 \pm 0.03$ \\
\hline 1603272950424941440 & 2.78 & 1603267143629157120 & Valenti \& Fischer 2005 & $0.11 \pm 0.03$ \\
\hline 116037376250217984 & 2.83 & 116037204451525376 & Valenti \& Fischer 2005 & $0.28 \pm 0.03$ \\
\hline 18565464288396416 & 2.86 & 19316224572460416 & Valenti \& Fischer 2005 & $-0.12 \pm 0.03$ \\
\hline 4187836934609063552 & 2.87 & 4187837450005193088 & Valenti \& Fischer 2005 & $0.05 \pm 0.03$ \\
\hline 1379500928055726848 & 2.91 & 1379500756257031808 & Valenti \& Fischer 2005 & $-0.69 \pm 0.03$ \\
\hline 581103581886377728 & 2.92 & 581100382135253760 & Valenti \& Fischer 2005 & $0.10 \pm 0.03$ \\
\hline 704966762213039488 & 2.99 & 704967037090946688 & Valenti \& Fischer 2005 & $0.31 \pm 0.03$ \\
\hline 3928040341460587904 & 3.04 & 3928040444539772800 & Valenti \& Fischer 2005 & $0.08 \pm 0.03$ \\
\hline 2029432043779954432 & 3.18 & 2029433521248546304 & Valenti \& Fischer 2005 & $0.21 \pm 0.03$ \\
\hline 3374633977170787328 & 1.51 & 3374633977170787072 & Ramírez et al. 2007 & $-0.96 \pm 0.05$ \\
\hline 3895404602964117504 & 1.52 & 3895404602962524544 & Mann et al. 2013c & $-0.16 \pm 0.03$ \\
\hline 238521872077340416 & 1.67 & 238521867779351296 & Mann et al. 2013c & $-0.01 \pm 0.03$ \\
\hline 248004472671505152 & 1.72 & 248004472671281664 & Mann et al. 2013c & $0.20 \pm 0.03$ \\
\hline 2258411902954640512 & 1.74 & 2258413380423390464 & Mann et al. 2013c & $0.05 \pm 0.04$ \\
\hline 2586715870164766336 & 1.79 & 2586715870164766464 & Mann et al. 2013c & $0.10 \pm 0.03$ \\
\hline 4418849515915901312 & 1.82 & 4418850954729363584 & Mann et al. 2013c & $-0.38 \pm 0.03$ \\
\hline 686996515965961728 & 1.84 & 686996756484129664 & Robinson et al. 2007 & $0.31 \pm 0.07$ \\
\hline 2462426800883156480 & 1.86 & 2462426800883134336 & Valenti \& Fischer 2005 & $0.12 \pm 0.03$ \\
\hline 3907643060733826432 & 1.87 & 3907643060733826560 & Mann et al. 2013c & $0.11 \pm 0.03$ \\
\hline 6272950819874104960 & 1.87 & 6272950029600121984 & Mann et al. 2013c & $0.15 \pm 0.03$ \\
\hline 716547127913595520 & 1.89 & 716546681236997888 & Casagrande et al. 2011 & $0.24 \pm 0.08$ \\
\hline 3630359628505968640 & 1.89 & 3630359589851275008 & Mann et al. 2013c & $-0.14 \pm 0.03$ \\
\hline 3977050728669175040 & 1.91 & 3977050728669174912 & Mann et al. 2013c & $0.46 \pm 0.03$ \\
\hline 1960631100090155264 & 1.96 & 1960631100090155008 & Robinson et al. 2007 & $0.03 \pm 0.07$ \\
\hline 238242871001896448 & 1.98 & 238195901239543424 & Casagrande et al. 2011 & $-0.40 \pm 0.08$ \\
\hline 1602355133093554560 & 2.00 & 1602402034136426752 & Casagrande et al. 2011 & $-0.17 \pm 0.08$ \\
\hline 263916742385357056 & 2.07 & 263916708025623680 & Valenti \& Fischer 2005 & $0.19 \pm 0.03$ \\
\hline 937667952870488704 & 2.07 & 937667952870489856 & Mann et al. 2013c & $-0.26 \pm 0.03$ \\
\hline 3419636575776307072 & 2.10 & 3419824248667573248 & Casagrande et al. 2011 & $-0.03 \pm 0.08$ \\
\hline 3120524882802287616 & 2.12 & 3120526738228080256 & Mann et al. 2013c & $-0.54 \pm 0.03$ \\
\hline 1760208512800938752 & 2.14 & 1760204939386236288 & Mann et al. 2013c & $-0.38 \pm 0.03$ \\
\hline 4595996885151094784 & 2.15 & 4595997263108222592 & Mann et al. 2013c & $-0.05 \pm 0.03$ \\
\hline 3912817529956869248 & 2.17 & 3912817907913991424 & Mann et al. 2013c & $-0.12 \pm 0.03$ \\
\hline 1583101688058584320 & 2.18 & 1583101791137799552 & Mann et al. 2013c & $-0.54 \pm 0.03$ \\
\hline 438829835272390784 & 2.19 & 438829629113960704 & Valenti \& Fischer 2005 & $0.06 \pm 0.03$ \\
\hline 4549331996760861696 & 2.20 & 4549335123497064576 & Mann et al. 2013c & $-0.09 \pm 0.03$ \\
\hline 1518618835782597888 & 2.21 & 1518617358313845888 & Mann et al. 2013c & $-0.05 \pm 0.03$ \\
\hline 1896261153909224064 & 2.23 & 1896449200461155712 & Robinson et al. 2007 & $0.21 \pm 0.07$ \\
\hline 3661352765349351552 & 2.23 & 3661352692334448128 & Mann et al. 2013c & $-0.03 \pm 0.03$ \\
\hline 4471250556472775296 & 2.23 & 4471250556472774400 & Mann et al. 2013c & $-0.66 \pm 0.03$ \\
\hline 1612283585653429632 & 2.26 & 1612283585653429760 & Mann et al. 2013c & $0.09 \pm 0.03$ \\
\hline 769364814652674944 & 2.28 & 769365192609825920 & Mann et al. 2013c & $-0.09 \pm 0.03$ \\
\hline 251738620318225792 & 2.44 & 251738448519542144 & Ramírez et al. 2007 & $-0.28 \pm 0.05$ \\
\hline 1391673002811376512 & 2.45 & 1391679909118786176 & Mann et al. 2013c & $-0.09 \pm 0.03$ \\
\hline
\end{tabular}


Table C1 - continued Stellar pairs and primary $[\mathrm{Fe} / \mathrm{H}]$ used for photometric $[\mathrm{Fe} / \mathrm{H}]$ relation

\begin{tabular}{ccccc}
\hline Gaia DR2 ID (s) & $B_{P}-R_{P}$ & Gaia DR2 ID (p) & [Fe/H] ref & {$[\mathrm{Fe} / \mathrm{H}]$ adopted } \\
\hline 1034999982042706048 & 2.53 & 1035000055055287680 & Mishenina et al. 2004 & $-0.16 \pm 0.07$ \\
3223332136283271296 & 2.53 & 3223331006703460352 & Bean et al. 2006a & $0.17 \pm 0.06$ \\
2384348242516852864 & 2.66 & 2384342302577019264 & Mann et al. 2013c & $-0.04 \pm 0.08$ \\
1416123117756120960 & 2.82 & 1416124285987232256 & Mann et al. 2013c & $-0.12 \pm 0.03$ \\
2005804153064109056 & 2.85 & 2005798350576886144 & Fuhrmann 2008 & $0.04 \pm 0.05$ \\
3641698032930553088 & 3.00 & 3641697998570813952 & Valenti \& Fischer 2005 & $0.56 \pm 0.03$ \\
1240498400329095168 & 3.11 & 1240498331609669120 & Ramírez et al. 2007 & $-0.50 \pm 0.05$ \\
2669364200719508352 & 3.30 & 2669361142702790272 & Valenti \& Fischer 2005 & $0.16 \pm 0.03$ \\
2411728182287010816 & 2.26 & HD 219617 & Ramírez et al. 2007 & $-1.28 \pm 0.17$ \\
\hline
\end{tabular}


Table D1. Nonlinear limb darkening coefficients from Claret (2017)

\begin{tabular}{|c|c|c|c|c|}
\hline TIC & $a_{1}$ & $a_{2}$ & $a_{3}$ & $a_{4}$ \\
\hline 410153553 & 1.479 & 0.174 & -0.928 & 0.472 \\
\hline 200322593 & 1.863 & -1.754 & 1.264 & -0.374 \\
\hline 92226327 & 1.589 & -1.152 & 0.657 & -0.152 \\
\hline 259962054 & 1.558 & -1.100 & 0.609 & -0.135 \\
\hline 348538431 & 3.672 & -5.444 & 4.411 & -1.347 \\
\hline 369327947 & 3.002 & -4.406 & 3.796 & -1.249 \\
\hline 141608198 & 3.261 & -4.842 & 4.096 & -1.317 \\
\hline 231702397 & 2.803 & -4.078 & 3.570 & -1.193 \\
\hline 98796344 & 3.066 & -4.665 & 4.129 & -1.389 \\
\hline 36724087 & 2.629 & -3.737 & 3.274 & -1.098 \\
\hline 158588995 & 2.422 & -3.290 & 2.861 & -0.957 \\
\hline 153065527 & 2.601 & -3.685 & 3.229 & -1.084 \\
\hline 307210830 & 2.917 & -4.373 & 3.869 & -1.302 \\
\hline 429358906 & 2.508 & -3.496 & 3.058 & -1.026 \\
\hline 118327550 & 2.512 & -3.520 & 3.087 & -1.037 \\
\hline 300710077 & 2.591 & -3.694 & 3.251 & -1.094 \\
\hline 35009898 & 2.322 & -3.115 & 2.715 & -0.911 \\
\hline 231728511 & 2.454 & -3.408 & 2.989 & -1.005 \\
\hline 413248763 & 2.640 & -3.809 & 3.361 & -1.132 \\
\hline 260708537 & 2.400 & -3.297 & 2.889 & -0.971 \\
\hline 150428135 & 2.357 & -3.205 & 2.804 & -0.942 \\
\hline 27649847 & 2.366 & -3.226 & 2.823 & -0.949 \\
\hline 370133522 & 2.431 & -3.373 & 2.963 & -0.997 \\
\hline 406941612 & 2.336 & -3.170 & 2.775 & -0.933 \\
\hline 259377017 & 2.574 & -3.683 & 3.252 & -1.096 \\
\hline 77156829 & 2.665 & -3.881 & 3.435 & -1.159 \\
\hline 220479565 & 3.015 & -4.772 & 4.370 & -1.504 \\
\hline 415969908 & 3.485 & -5.854 & 5.429 & -1.879 \\
\hline 141527579 & 3.547 & -6.087 & 5.725 & -1.999 \\
\hline 34068865 & 3.662 & -6.356 & 5.990 & -2.094 \\
\hline 29960110 & 3.689 & -6.433 & 6.079 & -2.128 \\
\hline 73649615 & 4.178 & -7.459 & 7.019 & -2.441 \\
\hline 260004324 & 4.342 & -7.781 & 7.298 & -2.530 \\
\hline 262530407 & 4.247 & -7.630 & 7.197 & -2.506 \\
\hline 271596225 & 4.525 & -8.332 & 7.922 & -2.774 \\
\hline 149788158 & 4.088 & -7.396 & 7.057 & -2.480 \\
\hline 425934411 & 2.687 & -4.134 & 3.849 & -1.335 \\
\hline 54962195 & 3.532 & -6.228 & 5.993 & -2.122 \\
\hline 12423815 & 3.393 & -5.919 & 5.699 & -2.020 \\
\hline 296739893 & 3.399 & -5.934 & 5.715 & -2.025 \\
\hline 151825527 & 3.103 & -5.267 & 5.074 & -1.801 \\
\hline 237920046 & 3.637 & -6.480 & 6.246 & -2.214 \\
\hline 219195044 & 4.137 & -7.619 & 7.353 & -2.604 \\
\hline 234994474 & 1.670 & -1.949 & 1.810 & -0.638 \\
\hline 33521996 & 2.284 & -3.134 & 2.788 & -0.929 \\
\hline 374829238 & 1.984 & -2.556 & 2.312 & -0.791 \\
\hline 44737596 & 2.149 & -2.875 & 2.575 & -0.868 \\
\hline 261257684 & 2.229 & -3.030 & 2.703 & -0.909 \\
\hline 359271092 & 2.508 & -3.569 & 3.149 & -1.043 \\
\hline 12421862 & 2.213 & -3.043 & 2.756 & -0.947 \\
\hline 70899085 & 4.248 & -6.917 & 5.904 & -1.852 \\
\hline 55488511 & 3.742 & -5.936 & 5.088 & -1.606 \\
\hline 219229644 & 3.596 & -5.652 & 4.851 & -1.531 \\
\hline 192826603 & 2.617 & -3.752 & 3.269 & -1.051 \\
\hline 32497972 & 1.823 & -2.211 & 1.985 & -0.657 \\
\hline 144700903 & 1.418 & -1.431 & 1.340 & -0.460 \\
\hline 351601843 & 2.120 & -2.828 & 2.532 & -0.833 \\
\hline 362249359 & 3.216 & -5.021 & 4.415 & -1.413 \\
\hline 237914496 & 4.173 & -7.011 & 6.189 & -1.989 \\
\hline 170849515 & 5.144 & -9.201 & 8.292 & -2.710 \\
\hline 220459976 & 5.150 & -9.182 & 8.248 & -2.685 \\
\hline 100608026 & 5.481 & -10.069 & 9.214 & -3.059 \\
\hline 322063810 & 5.019 & -9.160 & 8.444 & -2.812 \\
\hline 44647437 & 3.271 & -5.739 & 5.574 & -1.926 \\
\hline
\end{tabular}

Table D1 - continued Limb darkening coefficients

\begin{tabular}{|c|c|c|c|c|}
\hline TIC & $a_{1}$ & $a_{2}$ & $a_{3}$ & $a_{4}$ \\
\hline 261108236 & 2.771 & -4.830 & 4.877 & -1.733 \\
\hline 37749396 & 3.184 & -5.778 & 5.803 & -2.064 \\
\hline 165317334 & 2.436 & -4.170 & 4.313 & -1.549 \\
\hline 179985715 & 3.242 & -6.008 & 6.095 & -2.182 \\
\hline 33831980 & 2.889 & -5.229 & 5.357 & -1.921 \\
\hline 141527965 & 1.202 & -1.424 & 1.681 & -0.610 \\
\hline 229111835 & 1.276 & -1.586 & 1.836 & -0.661 \\
\hline 36734222 & 1.383 & -1.809 & 2.038 & -0.725 \\
\hline 254113311 & 1.652 & -2.370 & 2.551 & -0.888 \\
\hline 219338557 & 1.617 & -2.234 & 2.373 & -0.821 \\
\hline 50618703 & 1.580 & -2.170 & 2.328 & -0.808 \\
\hline 440887364 & 1.619 & -2.285 & 2.461 & -0.854 \\
\hline 101948569 & 1.667 & -2.443 & 2.644 & -0.919 \\
\hline 167600516 & 1.535 & -2.143 & 2.353 & -0.820 \\
\hline 210873792 & 1.578 & -2.258 & 2.474 & -0.863 \\
\hline 19025965 & 1.692 & -2.547 & 2.774 & -0.969 \\
\hline 251848941 & 1.576 & -2.286 & 2.519 & -0.882 \\
\hline 14165625 & 1.748 & -2.745 & 3.007 & -1.057 \\
\hline 38510224 & 1.616 & -2.431 & 2.685 & -0.943 \\
\hline 62483237 & 1.707 & -2.645 & 2.897 & -1.016 \\
\hline 158297421 & 1.728 & -2.685 & 2.923 & -1.021 \\
\hline 175532955 & 1.767 & -2.777 & 3.012 & -1.051 \\
\hline 280437559 & 1.877 & -3.038 & 3.280 & -1.147 \\
\hline 260417932 & 1.864 & -3.025 & 3.277 & -1.147 \\
\hline 201642601 & 1.870 & -3.048 & 3.303 & -1.157 \\
\hline 122613513 & 1.804 & -2.898 & 3.163 & -1.111 \\
\hline 201248411 & 3.659 & -6.702 & 6.491 & -2.171 \\
\hline 193641523 & 4.062 & -7.523 & 7.206 & -2.398 \\
\hline
\end{tabular}


Table E1. Summary of literature properties for already confirmed planets

\begin{tabular}{|c|c|c|c|c|c|c|c|}
\hline TOI & TIC & Name & $R_{P} / R_{\star}$ & $a / R_{\star}$ & $\begin{array}{c}\mathrm{i} \\
\left(^{\circ}\right)\end{array}$ & $\begin{array}{c}R_{P} \\
\left(R_{\oplus}\right)\end{array}$ & Reference \\
\hline 122.01 & 231702397 & TOI $122 \mathrm{~b}$ & $0.07500^{+0.003}$ & $25.20^{+1}$ & $88.40^{+0.60}$ & $2.720^{+0.180}$ & Waalkes et al. (2020) \\
\hline 129.01 & 201248411 & HIP $65 \mathrm{~A} \mathrm{~b}$ & $0.28700^{+0.0} .0$ & $5.29^{+0.05}$ & $77.18^{+0.92}$ & $22.800^{-6.6 .800}$ & Nielsen et al. (2020) \\
\hline 136.01 & 410153553 & LHS $3844 \mathrm{~b}$ & $0.06350_{-0.00090}^{+0.00090}$ & $7.11_{-0.03}^{+0.04}$ & $88.50_{-0.51}^{+0.51}$ & $1.303_{-0.022}^{+0.022}$ & Vanderspek et al. (2019) \\
\hline 175.01 & 307210830 & L 98-59 c & $0.03960_{-0.00080}^{+0.00110}$ & $22.50_{-1.40}^{+1.10}$ & $89.30_{-0.50}^{+0.40}$ & $1.350_{-0.070}^{+0.080}$ & Kostov et al. (2019) \\
\hline 175.02 & 307210830 & L 98-59 d & $0.04620_{-0.00300}^{+0.00300}$ & $37.40_{-1.20}^{+1.10}$ & $88.50_{-0.50}^{+0.20}$ & $1.570_{-0.140}^{+0.140}$ & Kostov et al. (2019) \\
\hline 175.03 & 307210830 & L 98-59 b & $0.02340_{-0.00080}^{+0.00090}$ & $16.20_{-1.00}^{+0.80}$ & $88.70_{-0.70}^{+0.80}$ & $0.800_{-0.050}^{+0.050}$ & Kostov et al. (2019) \\
\hline 178.01 & 251848941 & TOI- $178 \mathrm{~d}$ & $0.03623_{-0.00091}^{+0.00087}$ & 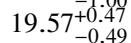 & $88.58_{-0.18}^{+0.20}$ & $2.572_{-0.078}^{+0.075}$ & Leleu et al. (2021) \\
\hline 178.02 & 251848941 & TOI-178 g & $0.04040_{-0.00180}^{+0.00190}$ & $42.13_{-1.06}^{+1.01}$ & $88.82_{-0.05}^{+0.04}$ & $2.870_{-0.130}^{+0.140}$ & Leleu et al. (2021) \\
\hline 178.03 & 251848941 & TOI-178 e & $0.03110_{-0.00120}^{+0.00110}$ & $25.87_{-0.65}^{+0.62}$ & $88.71_{-0.13}^{+0.16}$ & $2.207_{-0.090}^{+0.088}$ & Leleu et al. (2021) \\
\hline - & 251848941 & TOI-178 b & $0.01623_{-00}^{+0.0}$ & $8.61_{-0.22}^{+0.21}$ & $88.80_{-1.30}^{+0.80}$ & $1.152_{-0.070}^{+0.073}$ & Leleu et al. (2021) \\
\hline - & 251848941 & TOI-178 c & $0.02350_{-0.00130}^{+0.00150}$ & $12.23_{-0.31}^{+0.29}$ & $88.40_{-160}^{+1.10}$ & $\begin{array}{l}1.669_{-0.09}^{+0.1114} \\
114\end{array}$ & Leleu et al. (2021) \\
\hline - & 251848941 & TOI-178 f & $0.03220_{-0.00140}^{+0.00140}$ & $34.33_{-0.87}^{+0.82}$ & $88.72_{-0.07}^{+0.00}$ & $2.287_{-0.1108}^{+0.108}$ & Leleu et al. (2021) \\
\hline 237.01 & 305048087 & TOI $237 \mathrm{~b}$ & $0.06200_{-0.00200}^{+0.00200}$ & $34.70_{-2.90}^{+2.80}$ & $89.50_{-0.60}^{+0.40}$ & $1.440_{-0.120}^{+0.120}$ & Waalkes et al. (2020) \\
\hline 256.02 & 92226327 & LHS $1140 \mathrm{c}$ & $0.05486_{-0.00013}^{+0.000013}$ & $26.57_{-0.05}^{+0.05}$ & $89.92_{-0.09}^{+0.06}$ & $1.282_{-0.024}^{+0.020}$ & Ment et al. (2019) \\
\hline 270.01 & 259377017 & TOI-270 c & $0.05825_{-0.00058}^{+0.000079}$ & $27.01_{-1.58}^{+0.60}$ & $89.53_{-0.42}^{+0.30}$ & $2.420_{-0.130}^{+0.024}$ & Günther et al. (2019) \\
\hline 270.02 & 259377017 & TOI- $270 \mathrm{~d}$ & $0.05143_{-0.00074}^{+0.00074}$ & $41.56_{-0.83}^{+0.70}$ & $89.69_{-0.12}^{+0.16}$ & $2.130_{-0.120}^{+0.120}$ & Günther et al. (2019) \\
\hline 270.03 & 259377017 & TOI-270 b & $0.03000_{-0.00110}^{+0.000150}$ & $17.48_{-3.24}^{+1.49}$ & $88.65_{-1.40}^{+0.85}$ & $1.247_{-0.083}^{+0.089}$ & Günther et al. (2019) \\
\hline 442.01 & 70899085 & LP 714-47 b & $0.07510_{-0.00090}^{+0.00090}$ & $15.90_{-0.70}^{+1.04}$ & $87.30_{-0.20}^{+0.20}$ & $4.700_{-0.300}^{+0.080}$ & Dreizler et al. (2020) \\
\hline 455.01 & 98796344 & LTT $1445 \mathrm{~A} \mathrm{~b}$ & $0.04580_{-0.00110}^{+0.00120}$ & $29.60_{-2.50}^{+2.60}$ & $89.40_{-0.46}^{+0.40}$ & $1.380_{-0.120}^{+0.130}$ & Winters et al. (2019) \\
\hline 468.01 & 33521996 & HATS- 6 b & $0.17978_{-0.00077}^{+0.000710}$ & $13.65_{-0.15}^{+0.15}$ & $88.21_{-0.09}^{+0.09}$ & $11.190_{-0.210}^{+0.210}$ & Hartman et al. (2015) \\
\hline 551.01 & 192826603 & NGTS-1 b & $0.23900_{-0.05400}^{+0.10000}$ & $12.20_{-0.70}^{+0.15}$ & $85.27_{-0.01}^{+0.09}$ & $14.900_{-3.700}^{+6.280}$ & Bayliss et al. (2018) \\
\hline 562.01 & 413248763 & GJ 357 b & $0.03310_{-0.0}^{+0.0}$ & $22.31_{-0.90}^{+0.76}$ & $89.12_{-0.31}^{+0.01}$ & $1.217_{-0.083}^{+0.084}$ & Luque et al. (2019) \\
\hline 656.01 & 36734222 & WASP-43 b & $0.15880_{-0.00400}^{+0.00400}$ & $4.97_{-0.14}^{+0.140}$ & $82.11_{-0.09}^{+0.09}$ & $11.280_{-0.190}^{+0.0890}$ & Esposito et al. (2017) \\
\hline 700.01 & 150428135 & TOI-700 c & $0.05740_{-0.00260}^{+0.00320}$ & $47.80_{-2.60}^{+2.70}$ & $88.90_{-0.11}^{+0.08}$ & $2.630_{-0.230}^{+0.240^{\circ}}$ & Gilbert et al. (2020) \\
\hline 700.02 & 150428135 & TOI-700 d & $0.02620_{-0.00150}^{+0.00140}$ & $84.00_{-460}^{+4.70}$ & $89.73_{-012}^{+0.15}$ & $1.190_{-0.110}^{+0.110}$ & Gilbert et al. (2020) \\
\hline 700.03 & 150428135 & TOI-700 b & $0.02210_{-00}^{+0.0}$ & $34.80_{-1.90}^{+1.90}$ & $89.67_{-0.32}^{+0.23}$ & $1.010_{-0.087}^{+0.094}$ & Gilbert et al. (2020) \\
\hline 1067.01 & 201642601 & HATS- 48 A b & $0.11480_{-0.00200}^{+0.00200}$ & $11.33_{-0.05}^{+0.05}$ & $89.58_{-0.18}^{+0.18}$ & $8.970_{-0.170}^{+0.190}$ & Hartman et al. (2020) \\
\hline 1073.01 & 158297421 & HATS- 47 b & $0.17460_{-0.00140}^{+0.00140}$ & $13.98_{-0.15}^{-0.05}$ & $87.08_{-0.06}^{+0.06}$ & $12.520_{-0,160}^{+0.1100}$ & Hartman et al. (2020) \\
\hline 1130.01 & 254113311 & TOI-1130 b & $0.04860_{-0.00090}^{+0.00111}$ & $13.75_{-0.27}^{+0.31}$ & $87.98_{-0.46}^{+0.86}$ & $3.650_{-0.100}^{+0.100}$ & Huang et al. (2020) \\
\hline 1130.02 & 254113311 & TOI-1130 c & $0.21800_{-0.02900}^{+0.03700}$ & $22.21_{-0.43}^{+0.50}$ & $87.43_{-0.16}^{+0.16}$ & $16.800_{-2.500}^{+3.000}$ & Huang et al. (2020) \\
\hline
\end{tabular}

